\title{
INVESTIGATING PORE-FORMING PEPTIDES USING THE ATOMIC FORCE MICROSCOPE
}

\author{
A Dissertation \\ presented to \\ the Faculty of the Department of Physics and Astronomy \\ at the University of Missouri, Columbia

In Partial Fulfillment
of the Requirements for the Degree
Doctor of Philosophy \\ By \\ Anna Pittman \\ Dr. Gavin King, Dissertation Supervisor
}

May 2019 
The undersigned, appointed by the dean of the Graduate School, have examined the dissertation entitled

\section{INVESTIGATING PORE-FORMING PEPTIDES USING THE ATOMIC FORCE MICROSCOPE}

Presented by Anna Pittman

a candidate for the degree of Doctor of Philosophy, and hereby certify that, in their opinion, it is worthy of acceptance.

\section{Dr. Gavin King}

Dr. Peter Cornish

Dr. Ioan Kosztin

Dr. Suchi Guha 


\section{Acknowledgements}

It seems perfectly fitting to quote Isaac Newton in my dissertation in order to receive a PhD in Physics, but I promise it is more than just a platitude. Truly speaking, if I have seen further, it is only by standing on the shoulders of giants. I'll spare the reader the original Latin, but it appears to be a long-standing (no pun intended) truth in the field of science that it is only by benefiting from the works of those who came before us, that we can advance our scientific knowledge. To list every person whose work and dedication and care have led me to this point is perhaps a Sisyphean task, but let me attempt to highlight just a few.

Dr. Gavin King, my advisor, understandably deserves a huge portion of the praise, for it was through his mentoring that I got this far. In the world of graduate studies, sometimes finding a kind, caring advisor who places the students' needs above his own concern, is like winning the lottery. Even when the failed experiments piled higher and deeper, he remained a source of advice and hope that even the difficult stretches would one day yield fruitful results.

Dr. Krishna Sigdel somehow never showed an ounce of annoyance even though I must have interrupted his own work countless times to ask how a piece of equipment worked, or where a chemical was stored, or why the machine was acting a certain way. Instead, every time he would drop whatever he was doing to lend a hand, selflessly ready to teach me everything he knew.

To the members of my lab as a whole I can only thank en masse for the innumerable discussions and coffees shared. They helped me puzzle through many problems: from why my experiment failed to how gramophones really worked. They provided an invigorating and relaxed atmosphere which made it fun to come into work every day. 
Finally, I must thank my collaborators-Dr. William Wimley and Dr. Kalina Hristova. Not only did they provide me with fascinating peptides to study, they also were the source of many fruitful discussions. I am grateful for the opportunity to work with them and benefit from their wealth of biochemical knowledge. 


\section{Table of Contents}

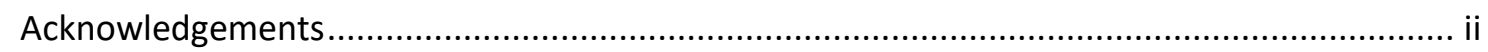

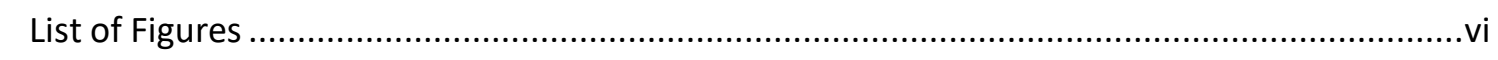

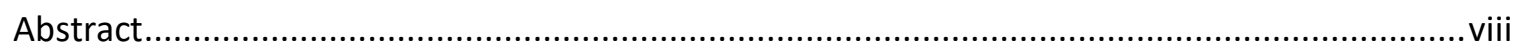

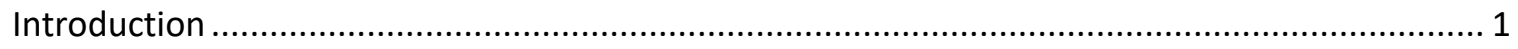

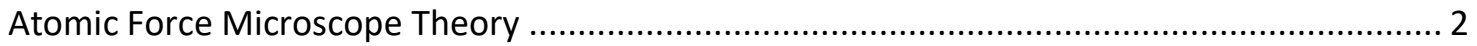

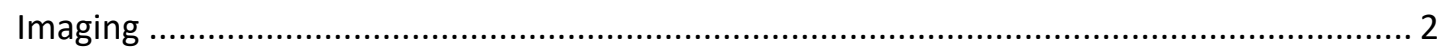

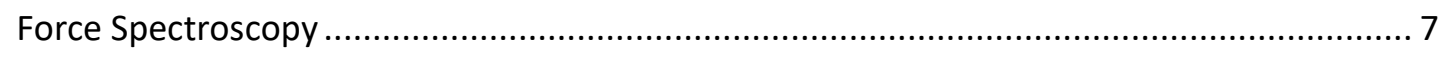

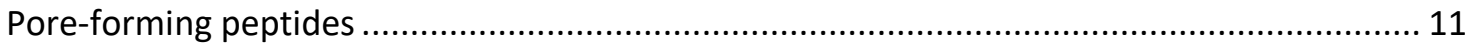

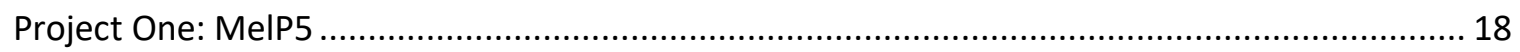

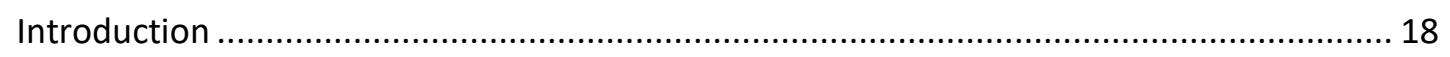

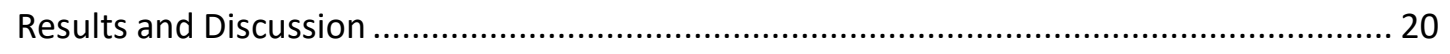

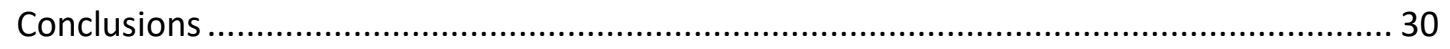

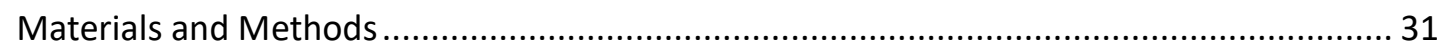

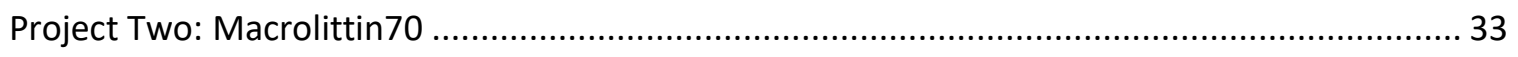

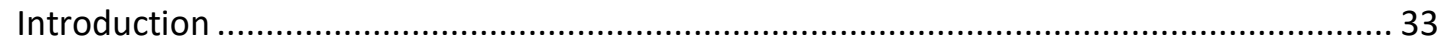

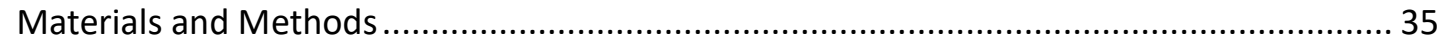

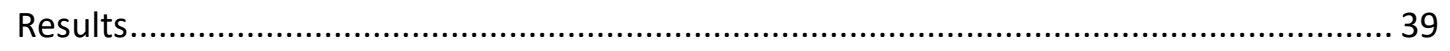

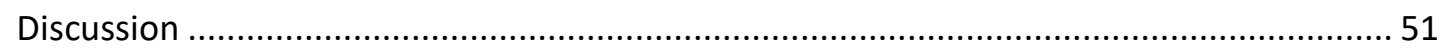

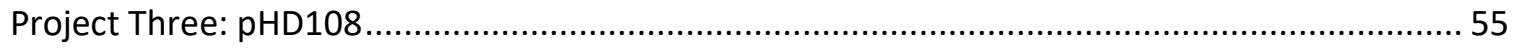

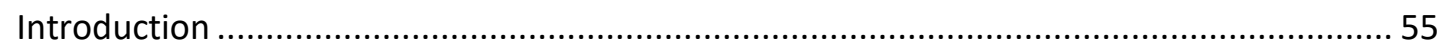

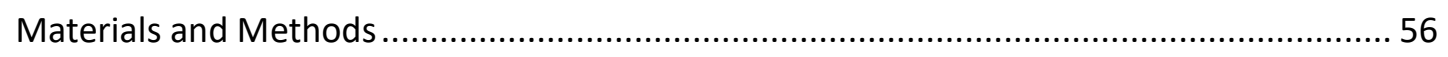

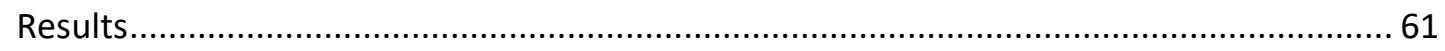

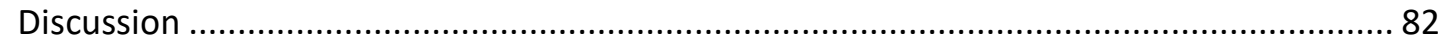

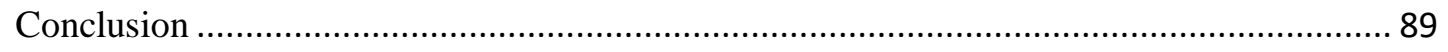

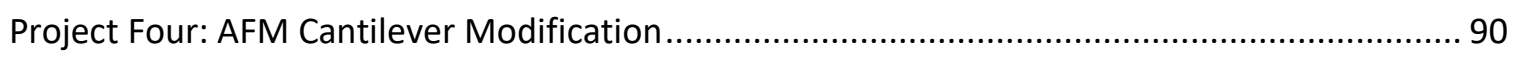

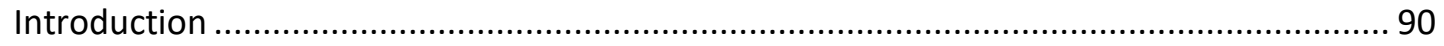

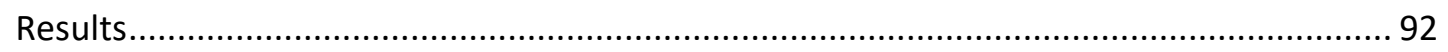

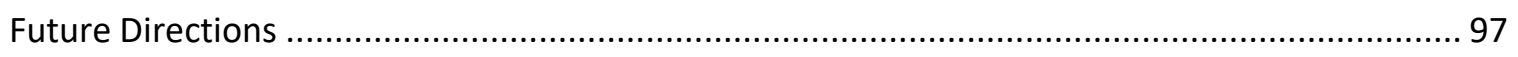

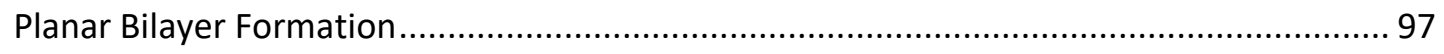

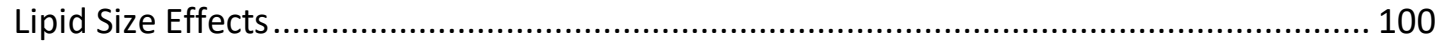


Pore-formation Mechanisms 102

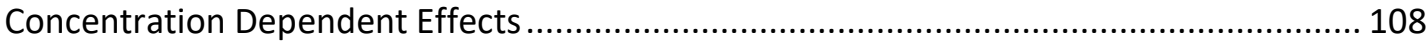

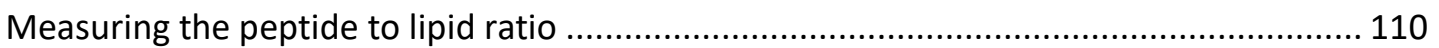

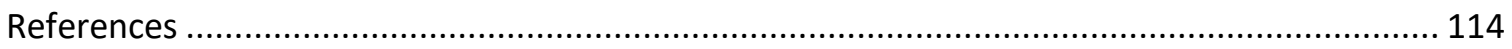

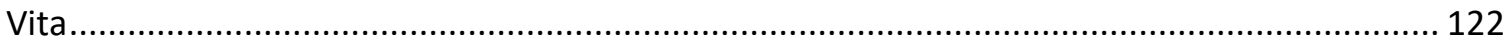




\section{List of Figures}

Figure 1. Scanning electron microscopy image of AFM tip ....................................................... 3

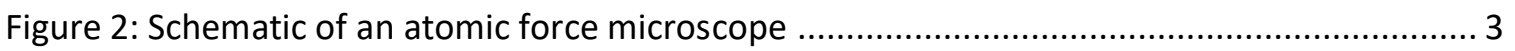

Figure 3 Cartoon depicting amplitude-modulated tapping mode............................................. 5

Figure 4. AFM image of human chromosome at varying set points.......................................... 7

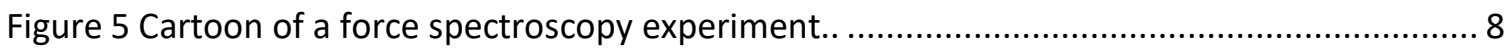

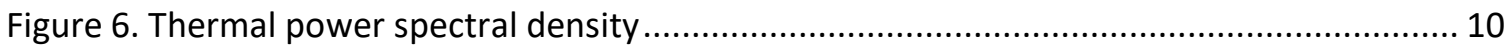

Figure 7. Linear region with fit of the force vs distance curve. ................................................. 11

Figure 8 Representative AFM images with no peptide and with $3.5 \mathrm{uM}$ of melittin................... 12

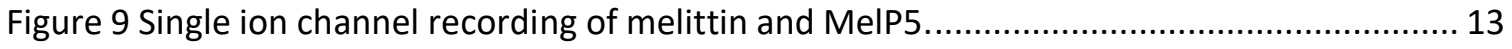

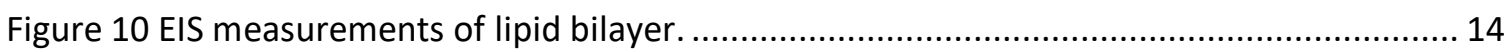

Figure 11 Attempts were made to investigate the effects of wild type melittin at varying peptide

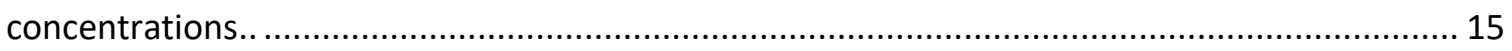

Figure 12 AFM imaging of amhelin-treated DLPC/DLPG supported bilayer............................... 16

Figure 13 Representative images of the membrane affected by MelP5.................................. 21

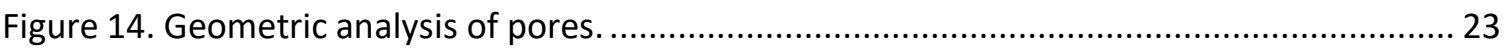

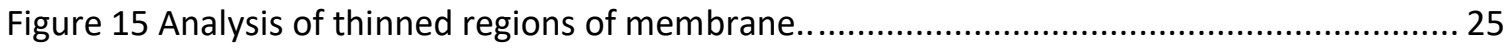

Figure 16. Integrated area histograms for both the pore-like features and the thinned features.

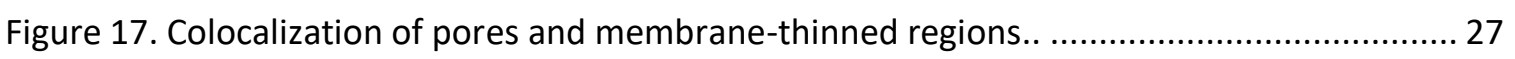

Figure 18. Dynamic transitions of MelP5-induced membrane topography. .............................. 28

Figure 19 The selection of macrolittins by synthetic molecular evolution. ................................. 38

Figure 20 Macrolittin induced leakage of a $40 \mathrm{kDa}$ dextran from POPC vesicles.. ....................... 42

Figure 21 Solution circular dichroism spectra of macrolittins and MelP5 at $10 \mathrm{uM}$..................... 44

Figure 22 Tryptophan fluorescence spectra of the macrolittins and MelP5............................ 46

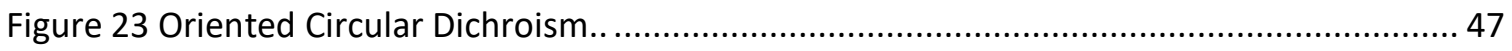

Figure 24 Membrane topography via atomic force microscopy. .............................................. 49

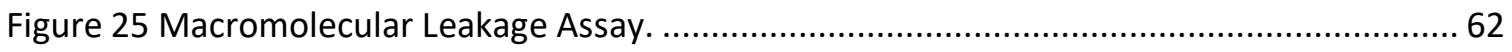

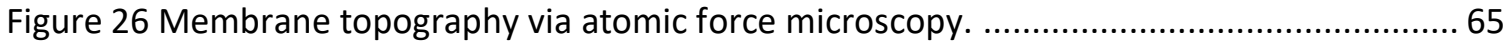

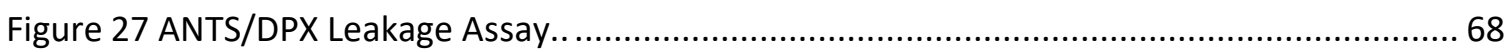

Figure $28 \mathrm{pHD}$ peptide binding to lipid bilayers, as followed by tryptophan fluorescence. ......... 70

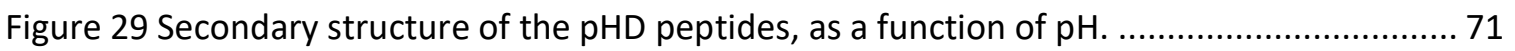

Figure 30 Comparison of pH50s for binding (BD), folding (CD), ANTS/DPX leakage (ANTS) and

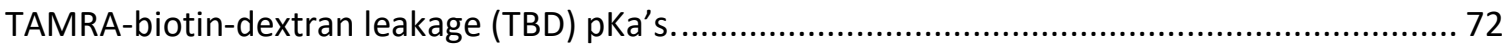

Figure 31 Comparison of binding and dextran leakage activity curves as a function of $\mathrm{pH}$ and P:L..

Figure 32 The activity of the pHD peptides depends on the number of bound peptides per

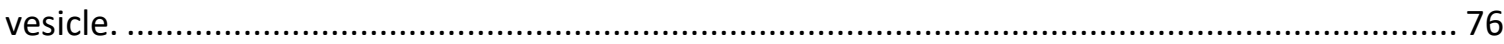

Figure 33 Quenching of tryptophan fluorescence by redistribution of pHD peptides.. ............... 78

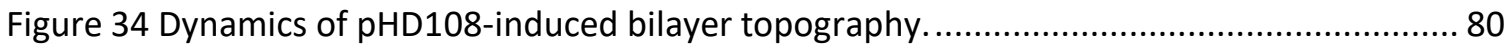

Figure 35 From left to right SEM images of cantilevers. ...................................................... 92 
Figure 36 Power spectral density data for cantilevers ......................................................... 94

Figure 37 Force precision at various bandwidths for modified and unmodified cantilever. ....... 95

Figure 38 FIB modified tips can achieve higher time resolution without sacrificing force

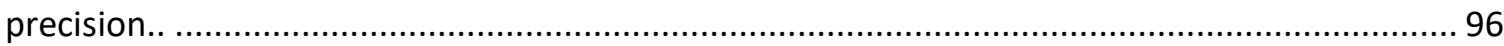

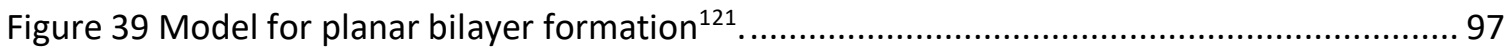

Figure 40. Preliminary results of fluorescently labeled POPC absorbed on a glass slide .............. 99

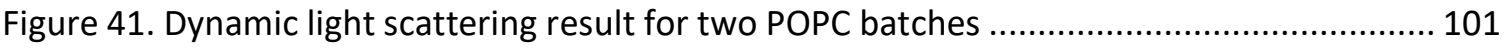

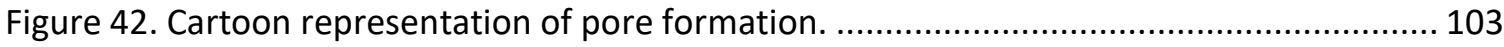

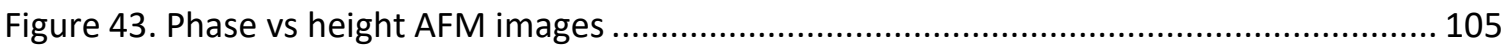

Figure 44. Height and phase images for both pHD108 and MelP5 pores................................ 106

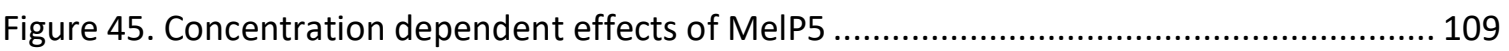

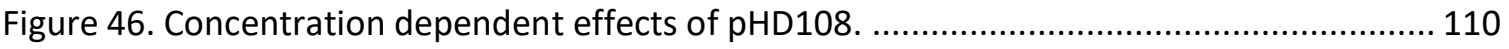

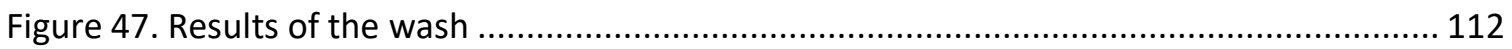




\begin{abstract}
In my projects, I used the Atomic Force Microscope (AFM) to image the effects of pore-forming peptides and I used the Focused Ion Beam (FIB) to modify AFM cantilevers to increase the precision of force spectroscopy measurements.

Self-assembly into stable membrane spanning pores makes MelP5 a promising candidate for future applications in the pharmaceutical arena. Despite significant interest, little is known about the mechanism by which MelP5 remodels the lipid bilayer upon binding. Using the AFM, I showed that MelP5 affects the bilayer in one of two ways: punctate depressions, or non-localized thinning. The peptide-effected bilayer was observed to reversibly exchange between membrane-thinned and pore states in an apparent dynamic equilibrium. Moreover, pores were found to colocalize with membrane-thinned regions, a novel observation that is consistent with the notion of cooperativity among membrane-bound peptides when forming pores.

Using MelP5 as a template, two high-throughput screens found other potent poreforming peptides. Out of these new potent pore-forming peptides, I studied two of them: Macrolittin70 and pHD108. Marcolittin70 was known to have high activity at pH 7, and had been shown to release larger macromolecules than MelP5 at low peptide to lipid ratios. Using the AFM I investigated the effects Macrolittin70 had on a POPC lipid bilayer compared it to the effects that MelP5 induced. The Macrolittin70-treated bilayer exhibited large and deep voids as well as numerous shallow defects. Some of these voids were deep and localized, suggestive of pores across the bilayer. MelP5-treated bilayers exhibited a single shallow defect mode under identical conditions.
\end{abstract}


pHD108 was developed to selectively interact with lipid bilayers under low pH conditions. Studies of pHD108 conducted on supported POPC bilayers at differing $\mathrm{pH}$ revealed $\mathrm{pH}$-dependent membrane distortions including punctate pore-like features. Additionally features found under low $\mathrm{pH}$ conditions exhibit varied temporal dynamics. Another way to investigate peptide-lipid interactions is to perform force spectroscopy experiments using the AFM. In order to lower drift and increase force precision, I used a FIB to modify commercially available AFM cantilevers. By reducing the cross sectional area of the cantilever, the hydrodynamic drag was reduced, thus increasing the force precision. Removing most of the gold coating on the cantilever increased the temporal stability. These modified cantilevers have already been put to use in the lab to measure peptide-lipid interactions. 


\section{Introduction}

Before the advent of single molecule techniques, bulk studies were used to investigate biological processes. While these bulk methods revealed many interesting properties about the biological systems, they were unable to parse out subtle intermediate stages. Any possibly relevant outliers in the data were washed out by the averaging that is unavoidable when doing a bulk measurement.

It was of interest, then, to develop techniques which could probe those quantities that were invisible to bulk measurements. In the 1980s technology caught up with that interest, and several novel single molecule techniques emerged. Ashkin et al showed in 1986 that it was possible to use light to trap nanoparticles ${ }^{1}$ — a technique now referred to as optical tweezers. Before the end of the decade that technique had been used to trap single live bacteria ${ }^{2}$. Optical tweezers are adept at probing the dynamics of molecular motors, such as kinesin ${ }^{3}$, and can study their activity on the single molecule level.

The 1980s saw not only the advent of optical tweezers but also atomic force microscopy (AFM), originally developed to characterize the topography of electrically insulating materials. Its ability to image materials that were not electrical conductive set it apart from its predecessor, the scanning tunneling microscope, and made it an ideal candidate for use in studying biological materials ${ }^{4}$. 


\section{Atomic Force Microscope Theory}

Imaging

The earliest scanning probe microscopes relied on a tunneling current which passed from the sample to the probe. This tunneling current could then be used to characterize the material. However, in order to create a current, the material being characterized had to be sufficiently conductive. AFM approached the problem of characterization from a different perspective. Instead of measuring a current, AFM measured the deflection of a sharp tip, with a radius of curvature on the order of nanometers (Figure 1). The sharp tip is scanned over the material of interest, and interactions with topographic features cause the tip to be deflected. Typically, that deflection is measured by reflecting a laser off of the back of a gold coated cantilever. The reflected laser beam then impinges upon a quadrant photo diode, which can measure the laser's deflection from an equilibrium point (Figure 2). This deflection from equilibrium is used to create topographical images of a surface with precision as low as sub-nm ${ }^{5}$ in the $\mathrm{z}$-dimension. In order to maintain biological relevance, the cantilever and tip can be submerged in buffer solution, allowing for the imaging of biological systems under physiological conditions. 


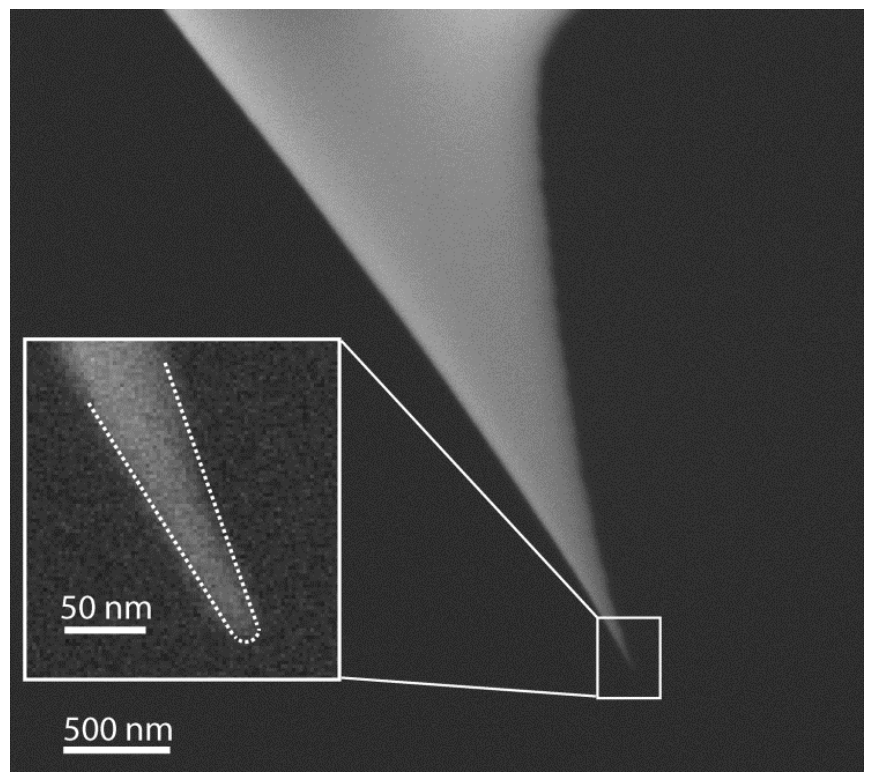

Figure 1. Scanning electron microscopy image of AFM tip. Here the measured radius of curvature was roughly $8 \mathrm{~nm}$.

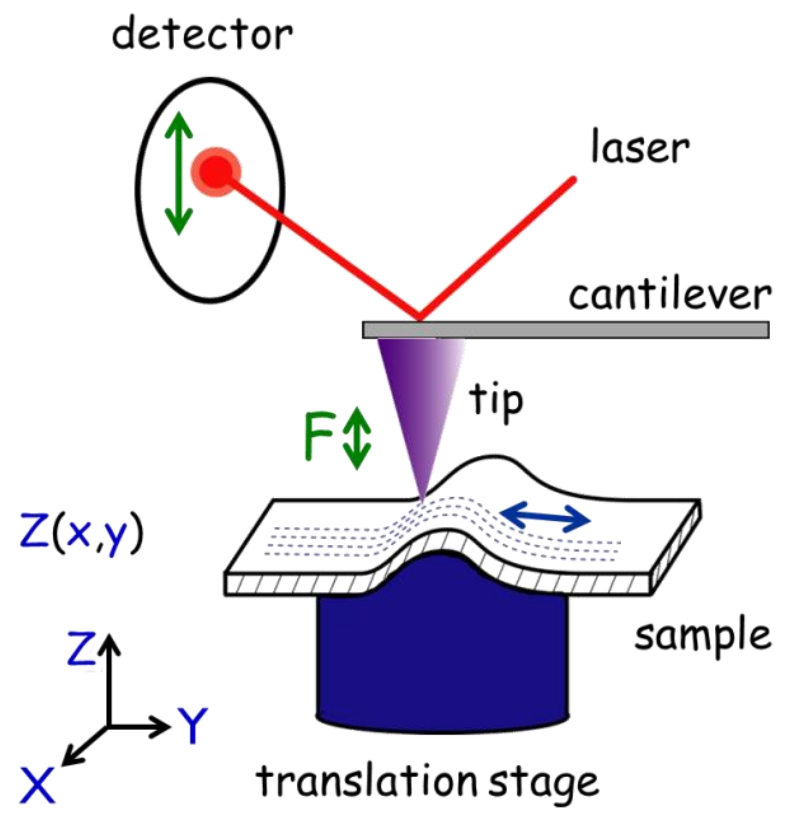

Figure 2: Schematic of an atomic force microscope 
The atomic force microscope can be operated in several imaging modes: contact mode, amplitude modulated tapping mode, and jumping mode.

In contact mode imaging, the tip is placed in contact with the sample. As the tip is scanned across the surface, a feedback loop monitors the deflection of the tip. When the tip encounters surface features, the cantilever is moved in the z-direction in order to keep a constant deflection. For positive features the feedback loop would cause the cantilever to be moved up, and down for negative features. This minute movement of the cantilever is facilitated by a piezoelectric motor. Piezoelectric materials are a special type of material whose dimensions can be manipulated by an applied voltage. In this manner, by measuring the z-motion of the cantilever required to keep a constant deflection, a topographical image of the sample is produced. This is the most straightforward method of imaging, but the sheer forces involved between the sharp tip and the sample are damaging to soft biological systems.

In order to minimize the damage imparted by imaging biologically relevant systems, tapping mode was developed. In tapping mode, the tip is driven at or near its resonance frequency, and is lowered towards the surface. Once again the small, precise movement required to drive the tip at its resonance frequency is achieved through a piezoelectric. As the tip oscillates, it has intermittent contact with the surface - in effect tapping the sample. As the tip scans the sample there are two possible feedback parameters, amplitude of the tip and frequency of the resonant peak. The one most often used is the amplitude of oscillation, as the frequency of the resonant peak is complicated by being submerged in a solution. As the tip encounters varying heights of the sample the amplitude of oscillation is either dampened — if the feature is higher than the tip —or 
enlarged - if the tip encounters a depression (Figure 3). The z-position of the cantilever is then adjusted so as to keep constant amplitude. By adjusting the set point- - the value of that constant amplitude - the user can control the amount of force the tip is imparting on the sample. A higher set point, that is, a set point that is closer to the free amplitude of the tip in solution, would result in a lower force imaging. A lower set point, that is, a number that is much smaller than the amplitude of the tip in free solution, would result in a high force imaging. Typical AFM images are taken with care to keep the tip sample force at or below $100 \mathrm{pN}$. This is calculated by comparing the constant imaging amplitude with the free amplitude of the cantilever in solution. The tip-sample force can have a large effect on the imaging process. Force microscopy experiments have shown that as low as $100 \mathrm{~s}$ $\mathrm{pN}$ is sufficient force to rupture a ligand from its binding site, such as biotin from streptavidin $^{6}$. Additionally, imaging of microtubules showed that the sample was deformed at tip-sample forces of $300 \mathrm{pN}^{7}$.
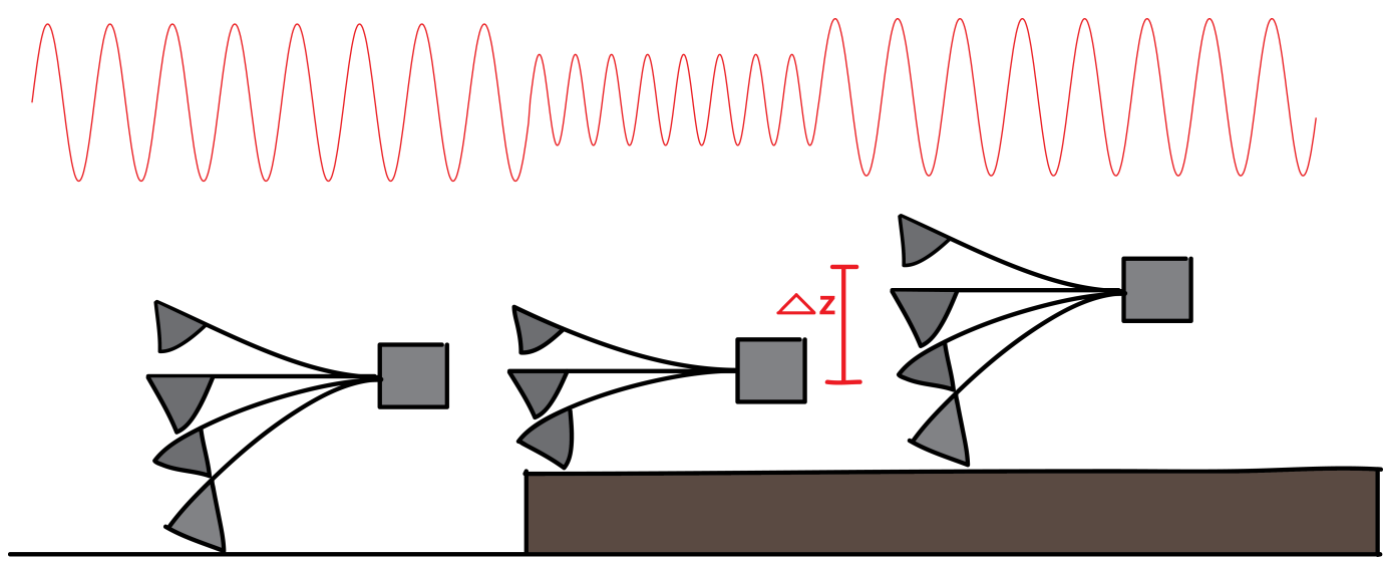

Figure 3 Cartoon depicting amplitude-modulated tapping mode. As the amplitude of oscillation signal impinging on the quadrant photo diode changes, the z-position of the tip is changed to maintain constant amplitude (and thus constant force on the sample).

Jumping mode, sometimes referred to as force spectroscopy imaging mode, is another method of taking an image with AFM. In jumping mode the tip is lowered 
towards and is pushed into the sample. In effect, a force spectroscopy measurement is made at each pixel. Out of the resultant force vs distance curve, the zero-force height (that is, the height measured just before force is applied) can be determined. This mode is ideal when imaging extremely soft materials which might disintegrate under higher forces. Tapping mode does minimize the tip sample force; however, the limiting factor to how fast the system can respond to changes in height is the piezoelectric element. Thus, there is the chance of increased forces when the tip first encounters a feature (exaggerated in Figure 3). Jumping mode, which very time consuming, can be a more precise way to image.

Jiao and Schaffer demonstrated the effect tip-sample force can have on height measurements ${ }^{8}$. They compared three modes of imaging: contact mode, tapping mode, and force spectroscopy imaging (i.e. jumping) mode, to see how each one effected the height measurement of human chromosomes. They used the force spectroscopy imaging mode to determine the zero-force height of the chromosomes and used that as a comparison to investigate the effect of various set points used in tapping mode.

Figure 4 shows those effects from changing the set point. In panel A the set point is $97 \%$ of the free amplitude (i.e. if the free amplitude were $300 \mathrm{mV}$ then the set point would be $291 \mathrm{mV}$ ). At this set point the tip is barely in contact with the chromosome and the image is not well formed. As the force increases (set point becomes smaller and smaller) the height measurements change. By panels C\&D the set point appears close to ideal, as the line scans match the dashed line (which come from the zero-force height image). As the force increases past that point (panels E\&F), the chromosome is deformed, and the height readings decrease. 


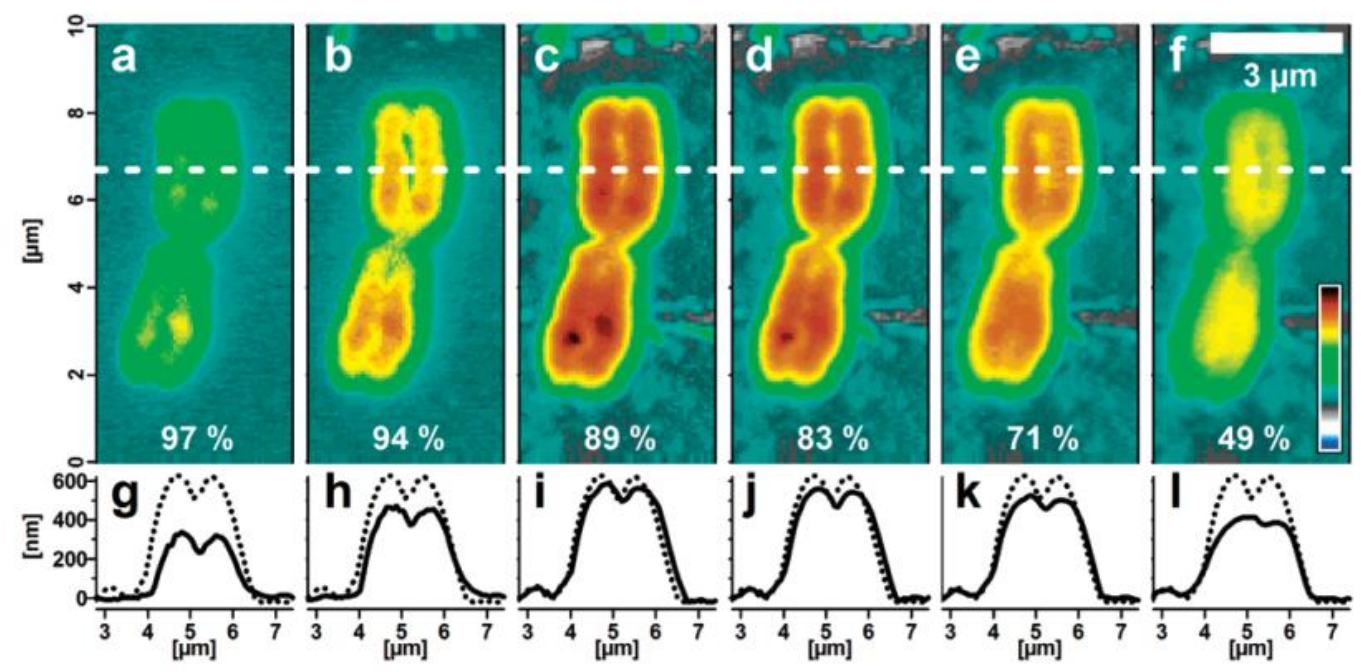

Figure 4. AFM image of human chromosome at varying set points. (A-F) height images of chromosomes. (G-I) Individual line scans as compared to a zero-force image (dashed) ${ }^{8}$.

As expected, contact mode had a similar result to tapping mode: larger forces lead to larger sample deformation. Thus it is important to use a set-point which is just low enough to stay in contact with the sample, but isn't so low (i.e. so high force) as to deform that sample. While taking an image in tapping mode, one can vary the set-point minutely during imaging to ensure that the lowest imaging force is applied.

\section{Force Spectroscopy}

While imaging is a common mode to investigate biological systems, it is not the only use for AFM. Single molecule force spectroscopy experiments investigate the energetic landscape between two interacting species - for example, between lipid bilayers and peptides. 


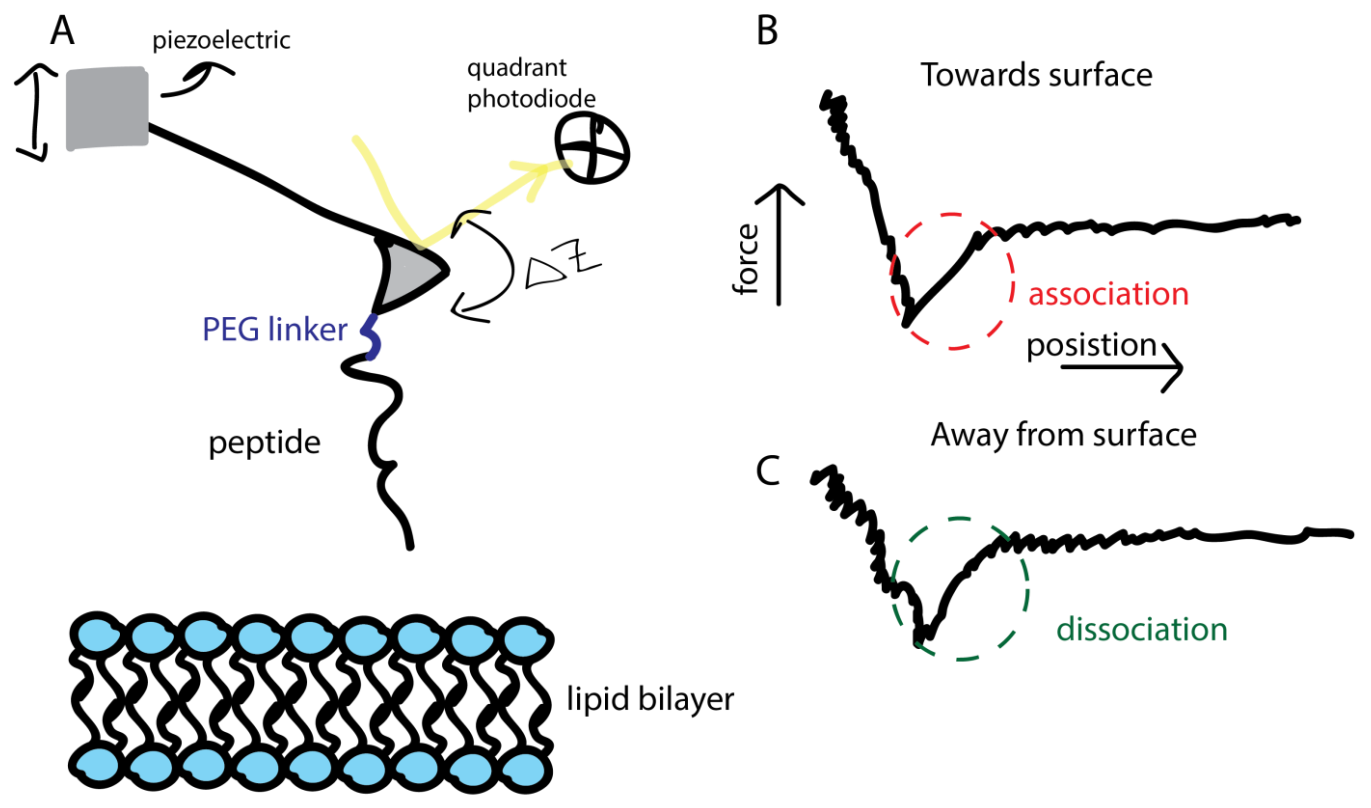

Figure 5 Cartoon of a force spectroscopy experiment. A: After linking the peptide of interest to the tip, the cantilever is lowered towards a lipid bilayer. B: When the tip gets close to the surface there is an association event in the force vs position data. C: As the tip is retracted from the surface, there is a dissociation event as the peptide leaves the lipid bilayer.

The first step in a normal force spectroscopy experiment is to functionalize the tip with the species of interest ${ }^{9}$. Typically this is done by coating the tip with a linker, such as PEG, and then covalently attaching the peptide to the PEG linker. After the tip is functionalized, it is lowered towards the surface, which can be covered in a lipid bilayer (Figure 5A). The deflection of the tip is closely monitored as it approaches the surface. While there are no forces acting on the cantilever, the deflection remains close to zero. As the peptide interacts with the lipid, it causes a deflection of the cantilever. First, when the peptide comes into close contact with the lipid bilayer, the association force is measured - the force which is caused by the peptide inserting into the lipid membrane (Figure 5B). Then, as the tip is retracted, the disassociation, or rupture force, is measured 
(Figure 5C). From these events the fundamental properties of the lipid-peptide interaction can be studied ${ }^{10}$ and the energetic landscape can be determined.

As commercially sold, AFM cantilevers are not optimized for force spectroscopy, experiments. Therefore it is of interest to modify the cantilever to improve its use in force spectroscopy. Following published work $^{11}$, we attempted to make such modifications, which will be detailed Project Four. Here I would like to provide a little background on one of the metrics used to measure the effects of the cantilever modification, the spring constant of the cantilever.

The most common way to measure the spring constant of an AFM cantilever is to use the thermal noise method ${ }^{12}$. In short, the AFM cantilever can be modeled as a harmonic oscillator fluctuating in response to the thermal noise due to the aqueous environment it is submerged in. The Hamiltonian for such a situation is given by Eq. 1 below.

$$
H=\frac{p^{2}}{2 m}+\frac{1}{2} m w_{0}^{2} x^{2}
$$

Here, $p$ is momentum, $m$ is the oscillating effective mass, $w_{0}$ is the resonant frequency, and $x$ is the displacement. Next, we invoke two equations: the equipartition theorem (Eq. 2) and the definition of the resonance frequency for a harmonic oscillator (Eq. 3).

$$
\begin{gathered}
\left\langle\frac{1}{2} m w_{0}^{2} x^{2}\right\rangle=\frac{1}{2} k_{B} T \\
w_{0}^{2}=\frac{k}{m}
\end{gathered}
$$


Putting these equations together, if one could measure the mean-square spring displacement, one could get a value for the stiffness of the cantilever (Eq. 4). The meansquare spring displacement is found by fitting the thermal noise curve of the cantilever.

$$
k=\frac{k_{B} T}{\left\langle x^{2}\right\rangle}
$$

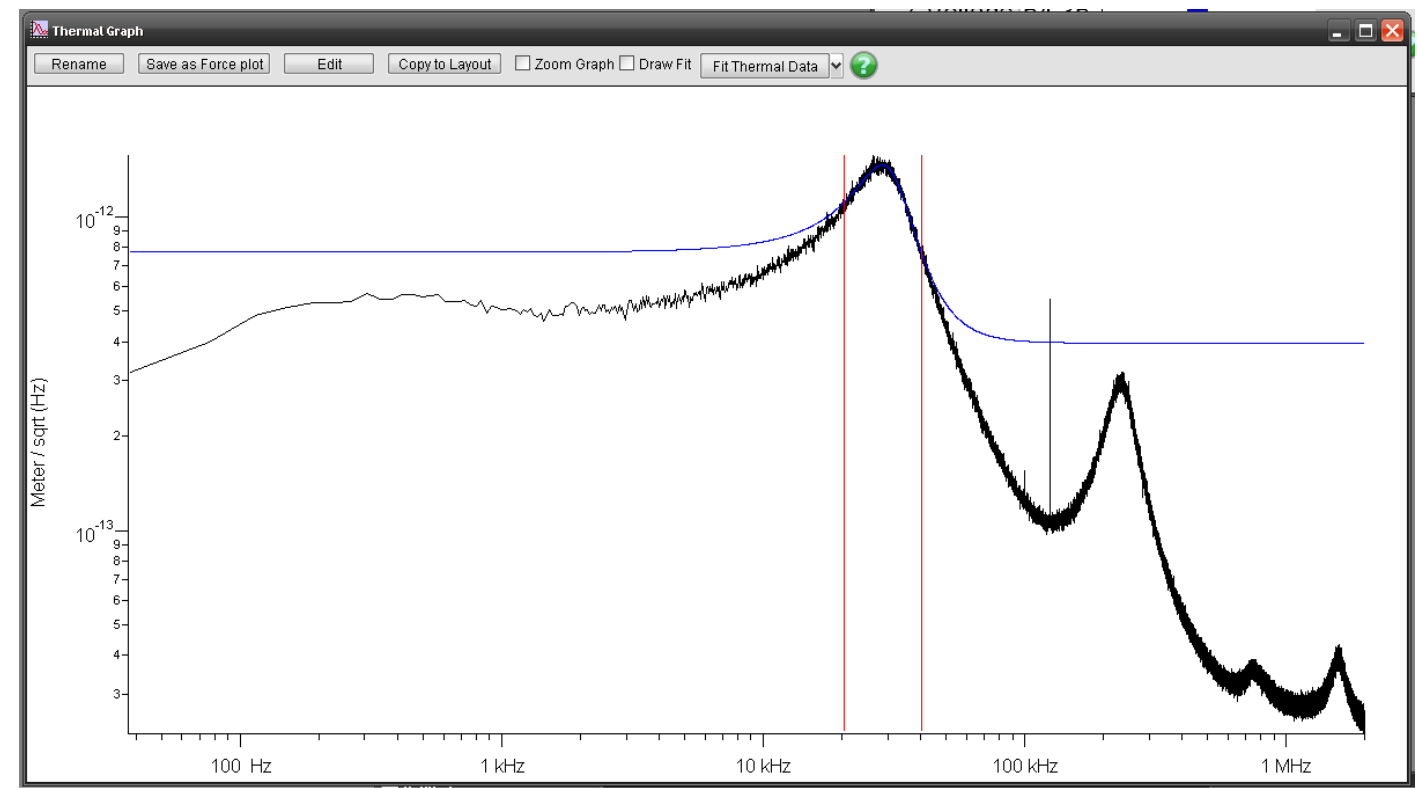

Figure 6. Thermal power spectral density

Figure 6 shows the thermal noise, referred to as the power spectral density (PSD). A PSD curve is effectively an auto-correlation curve and shows the deflection of cantilever away from equilibrium as a function of sampling frequency ${ }^{13}$. In order to find the spring constant the first resonant peak is fit, and the area under the curve corresponds to the mean square displacement ${ }^{12,14}$. However, the PSD data by default has units of volts instead of distance for displacement. Thus, before the spring constant can be calculated, the conversion factor between volts and meters for displacement of the cantilever must be determined. This is determined by doing a single force curve, that is, by pushing the tip into the surface and then retracting. The linear region of the resultant force vs distance 
curve is fit (Figure 7) and the inverse of the slope is used to convert the displacement into meters.

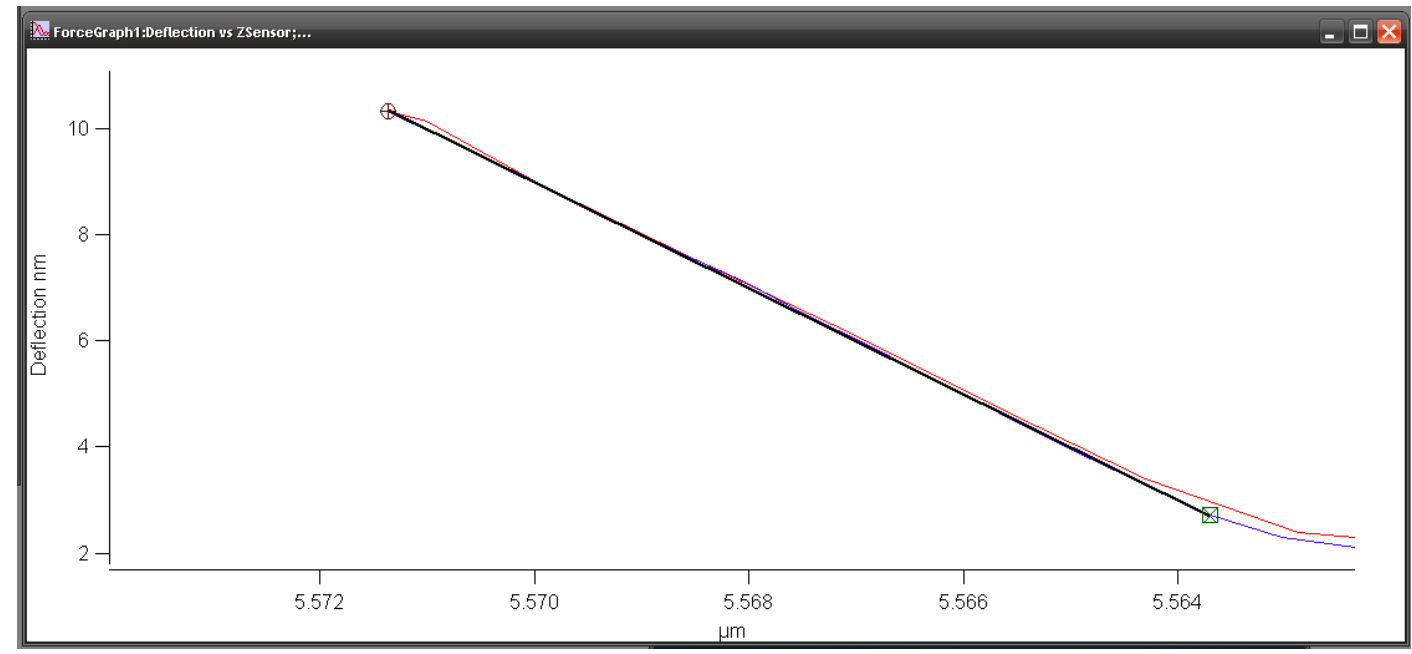

Figure 7. Linear region with fit of the force vs distance curve.

\section{Pore-forming peptides}

Peptides, small chains of amino acids which lack tertiary or quaternary structure, can self-assembly into oligomers and insert themselves into lipid membranes. These interactions destabilize the lipid membrane and can cause the creation of pores. Investigating these behaviors with methods such as AFM can be difficult as diffusion, and pore dynamics, can occur faster than the temporal resolution of the machine. Some work has been done in the area, but few papers manage to image clear pores; many report only large-scale destruction or disruption of the lipid bilayer. 
Melittin is one of the most widely studied antimicrobial peptides in the field, due to its relative simplicity and availability, and there are a few AFM studies which have attempted to elucidate the mechanism of membrane perturbation induced by melittin.

Shaw, et al, investigated the effects of melittin on supported lipid bilayers using $\mathrm{AFM}^{15}$. Figure 8 shows some of their results. On left is an AFM image of a DSPC/DOPC lipid bilayer with no melittin and on right is an image of a sample with $3.5 \mathrm{uM}$ of melittin. There is a clear difference between the two conditions. However, since melittin is not believed to create stable pores, it is unsurprising that the only thing that is seen is complete lipid destruction.
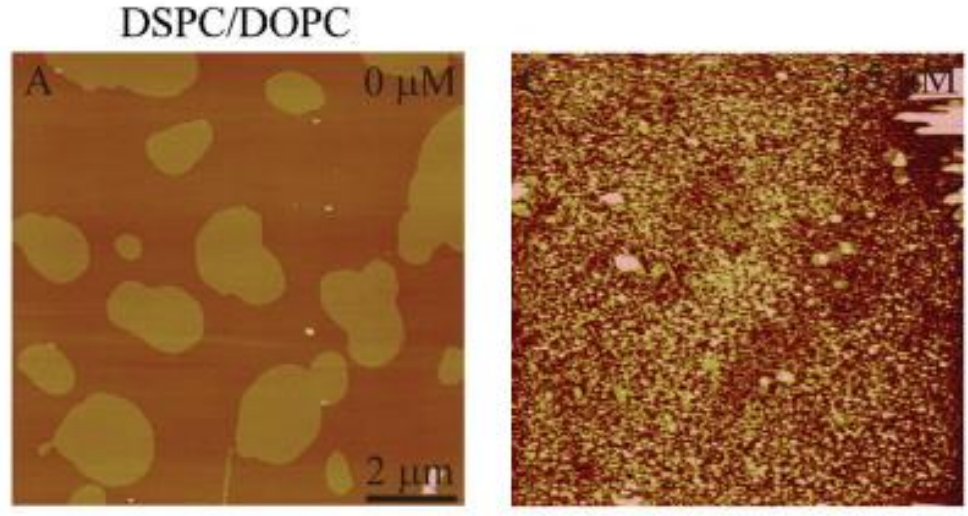

Figure 8 Representative AFM images with no peptide (left) and with $3.5 \mathrm{uM}$ of melittin (right). No pore-like structure is seen, only destruction ${ }^{15}$.

It should be noted that one paper claims to have used AFM to image pores induced by melittin in DLPC supported lipid bilayers ${ }^{16}$. However, the defects observed by the group were large (radius $~ 3.8 \mathrm{~nm}$ ) and grew as a function of imaging time ${ }^{16}$. Many papers using other methods dispute the idea that melittin creates a stable, transmembrane pore ${ }^{17}$. Fennouri, et al, investigated the effects of Melp5, a mutant peptide 
synthetically evolved from melittin, in comparison with melittin in POPC/Cholesterol bilayers using single ion channel recordings ${ }^{18}$.
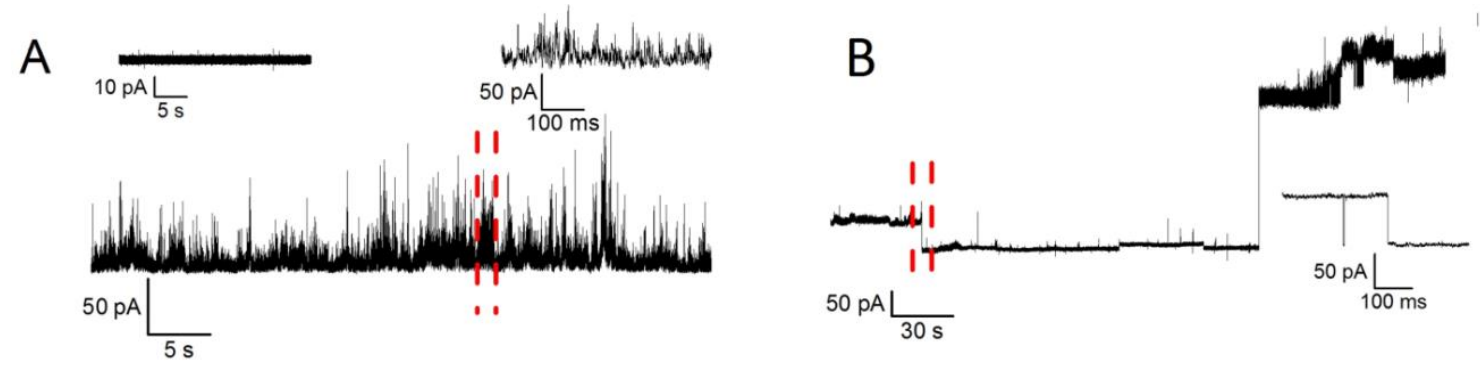

Figure 9 Single ion channel recording of melittin (A) and MelP5 (B) ${ }^{18}$.

As seen in Figure 9, the current traces for melittin (A) and MelP5 (B) look drastically different. Single ion channel recording experiments involve placing electrodes on either side of a lipid bilayer and measuring the current across it. Since lipid membranes naturally have a resistance on the order of Giga Ohms, very few ions are permitted to flow across the membrane. Thus, when the membrane is perturbed by peptides, any small change in current can be amplified and measured. Figure 9A shows that there are many short-lived current spikes, which is not indicative of stable pores. Stable pores would allow current to flow across the membrane for longer periods of time. MelP5 also showed traces of short-lived current spikes (not shown). However, it did showed stable pore behavior, as seen by the step function behavior of the current trace Figure 9B. Here, there are clear steps in the current vs time trace, and flat lines at high current — which occurs when a stable pore (or pores) exists in the lipid bilayer.

Wiedman, et al, used electrical impedance spectroscopy (EIS) and vesicle leakage studies to argue that melittin does not need to form a transmembrane pore in order to 
affect the permeability of the membrane ${ }^{17}$. EIS is a technique which can be used to study the resistance and capacitance of a lipid bilayer, similar to single ion channel recordings but over a larger scale.

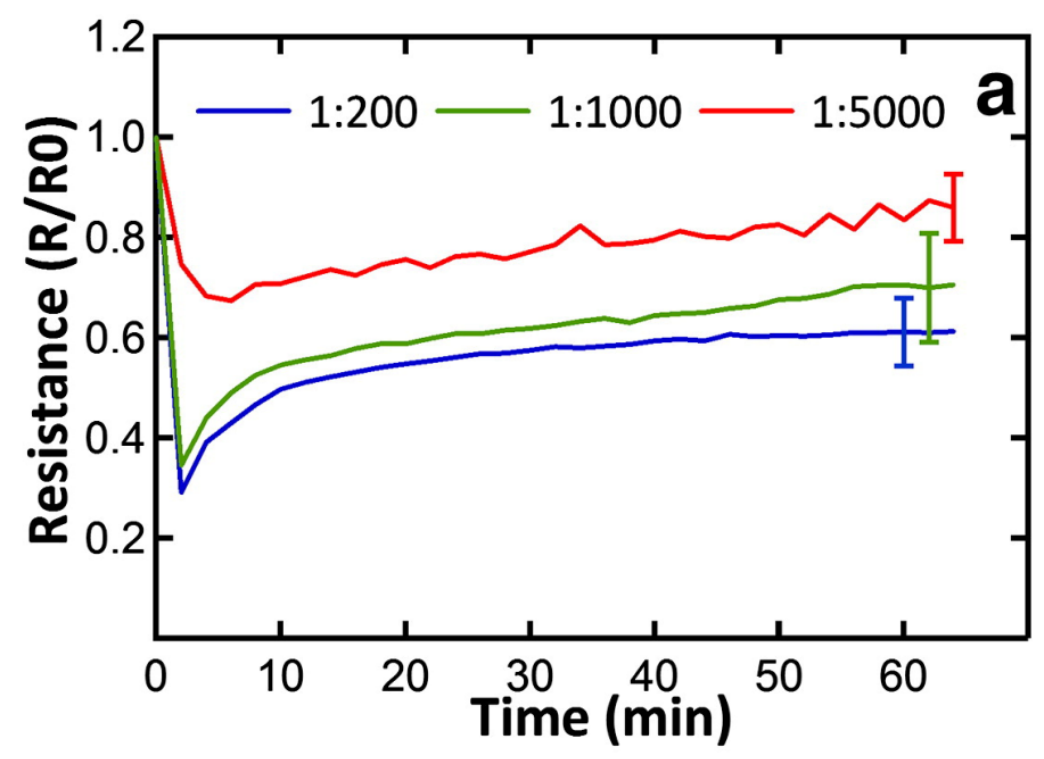

Figure 10 EIS measurements show that the resistance of the lipid bilayer decreases after addition of melittin for three different peptide to lipid ratios ${ }^{17}$.

As seen in Figure 10, the resistance of the bilayer, which was a mixture of POPC/Cholesterol, decreased after the addition of melittin. Three peptide to lipid ratios were tested. In all cases the lipid membrane experienced an initial decrease in resistance, followed by a recovery period. The other method they used to investigate the behavior of melittin was dye-release assays. In a dye-release assay a dye of a known size is encapsulated in a lipid vesicle. Release of the dye is monitored (typically by a fluorescence measurement) after addition of melittin. Weidman et al showed that the dye was released for certain peptide to lipid ratios, showing that melittin can perturb a lipid membrane enough to release cargo, even if it does not form a stable, transmembrane pore $^{17}$. 

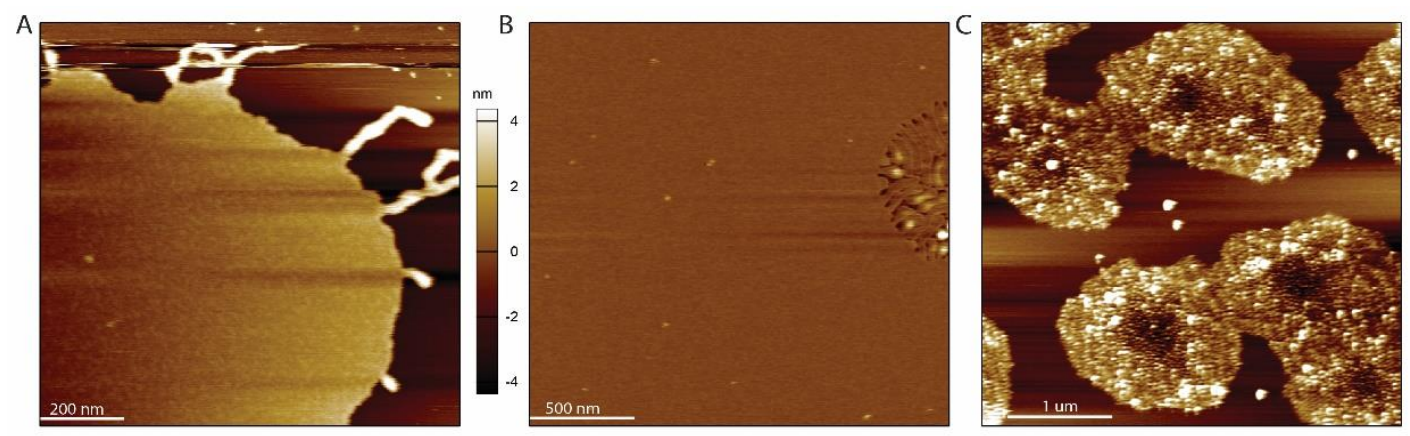

Figure 11 Attempts were made to investigate the effects of wild type melittin at varying peptide concentrations. A) $2.5 \mathrm{uM}$, B) $4 \mathrm{uM}$ and C) $6.2 \mathrm{uM}$. Though evidence of peptideinduced bilayer remodeling (and hence of peptide binding the bilayer) can be observed in all of these images (for example, the finger-like protrusions in (A), the spherical distortion on the right side of (B) and the overall destruction of the bilayer at the highest peptide concentration shown (C)), none of the trials resulted in the formation of stable pores.

Since melittin does not appear to form stable pores it does not lend itself to imaging methods such as AFM. Figure 11 shows my own efforts to image pore-like effects induced by melittin. Panel A, which has the lowest peptide concentration, shows odd finger-like protrusions, but the bilayer itself remains smooth. Panel B, which has a slightly higher peptide concentration, shows unusual perturbations but no pores. And panel C, which has the highest peptide concentration, shows the complete destabilization of the bilayer. Much effort was spent attempting to optimize the experimental protocol to try and induce pore formation. However, regardless of the incubation method, peptide concentration, or buffer composition, no pore-like features were observed.

While melittin does not seem to create distinct pores, there are few papers which image the effects of peptides which are believed to do so ${ }^{19-20}$.

Rakowska, et al, used the AFM to image the effects of a de nova peptide on a DLPC/DLPG supported lipid bilayer ${ }^{19}$. Figure 12 shows their results, which include both pores and large-scale destruction of the bilayer. Their studies show that the novel peptide (amhelin) formed pores in the lipid bilayer. These pore-like features then grew as a 
function of time until the bilayer was fully dissolved. However, they did not quantify the size, structure, or precise temporal dynamics of the pores. Rather, they only detailed the eventual destruction of the bilayer.
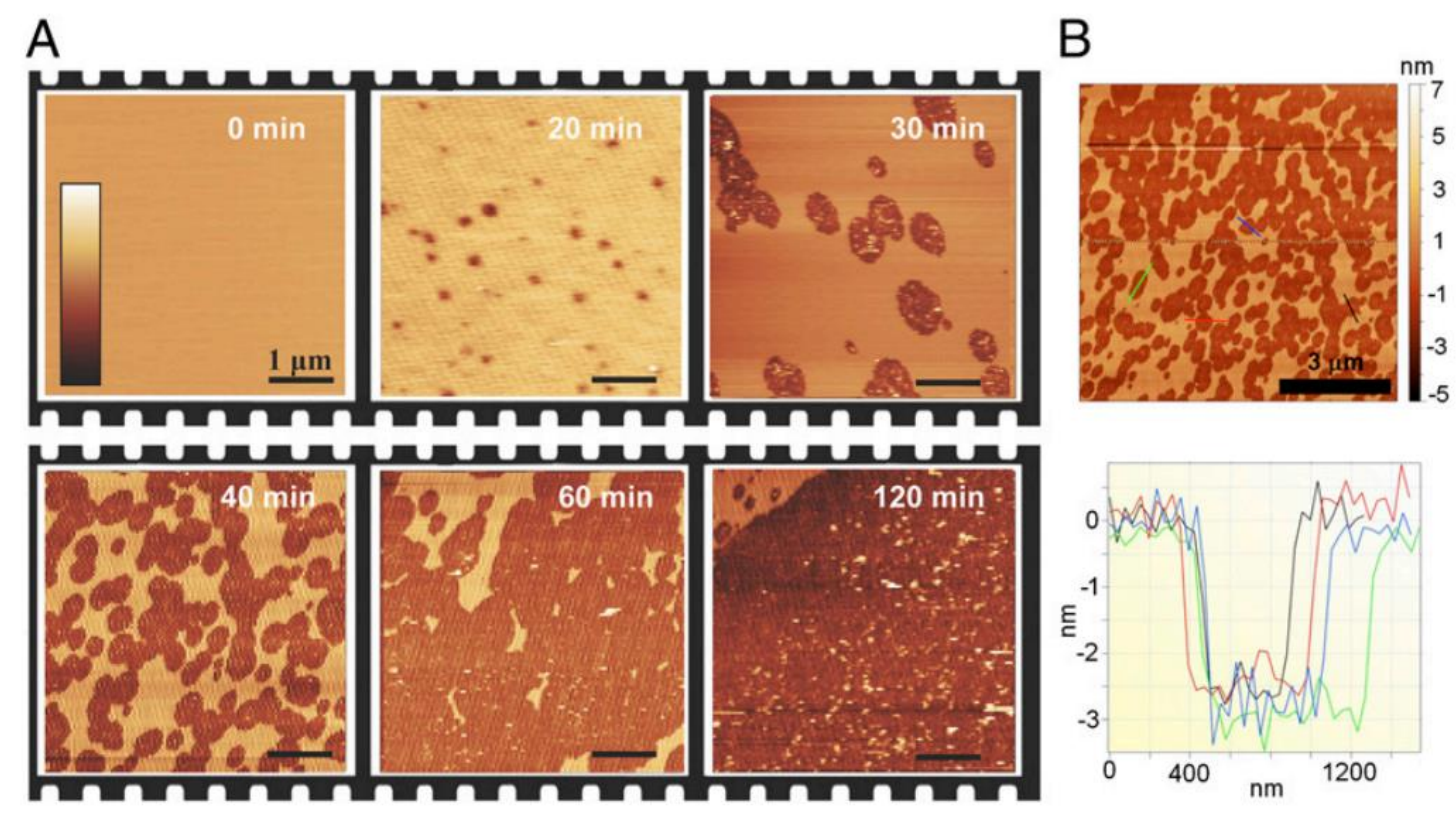

Figure 12 AFM imaging of amhelin-treated DLPC/DLPG supported bilayer. Peptide-induced defects grow as a function of time before dissolving the entire bilayer ${ }^{19}$.

Thus, even with peptides that do appear to make stable pores, there is a dearth of detailed imaging studies of their structure and any associated temporal dynamics. AFM fills an important experimental void, because it allows the structure and dynamics of these pores to be studied individually and in near native conditions (i.e. at room temperature and physiologically relevant salt concentrations). Therefore, my work appears to be both novel and needed to closely examine the effects of pore-forming peptides interacting with a lipid bilayer. This question of pore structure and dynamics becomes more pressing with the discovery of mutant peptides, three of which will be examined in detail in the coming chapters, which are incredibly potent and appear to 
form long-lasting, stable, transmembrane pores ${ }^{21-23}$ and which may eventually find clinical applications. 


\section{Project One: MelP5}

\section{Conformations and Dynamic Transitions of a Melittin Derivative That Forms Macromolecule-Sized Pores in Lipid Bilayers}

Anna E. Pittman, Brendan P. Marsh, and Gavin M. King

Langmuir 201834 (28), 8393-8399

\section{Introduction}

Polypeptide chains that bind to and subsequently form pores in membranes have garnered significant attention in fields ranging from fundamental biophysics to biotechnology. ${ }^{24}$ Such pore-forming peptides have significant promise in medicine as direct antimicrobial agents, as well as for drug delivery vehicles, including for targeted cancer therapy. ${ }^{25-27}$ Notwithstanding their potential, the complex modes of action and dynamic structure of these lipophilic peptides remain poorly understood. To develop more effective therapies and to inform development of novel pore-forming peptides, a more quantitative and mechanistic understanding of peptides interacting with near-native membranes is needed. ${ }^{10,28-29}$

Pore formation by lipophilic peptides has been investigated by several methods, including circular dichroism, lamellar x-ray diffraction, and surface-induced fluorescence. ${ }^{30-31}$ A model system for these studies is the melittin peptide, a chain of 26 amino acids that is the primary toxic component of Honey Bee venom. Melittin is widely known to affect cell membranes by forming pores, but its effect is short-lived, owing to the inherent instability of the pores. ${ }^{17,32-34}$

Wild-type melittin is known to have a bimodal interaction with a lipid bilayer; it can exist either in a surface-bound state, with its backbone approximately parallel to the membrane surface, or in an inserted state, forming pores that span the membrane. An 
amphipathic helix arises when melittin contacts the membrane. ${ }^{35-37}$ Furthermore, evidence shows that the percentage of peptides in each state is a function of the peptideto-lipid ratio. ${ }^{38}$ At low peptide concentrations, the majority of peptides associate with their backbones parallel to the membrane; this binding can locally displace lipid head groups, causing a thinning of the membrane (a few Angstroms for wild-type melittin). ${ }^{31}$ At higher peptide concentrations, the amino acid chains are thought to insert themselves into the membrane and create transmembrane pores.

To improve upon the shortcomings of wild-type, a variant of melittin, MelP5, was synthetically evolved to create stable pores with radii large enough to pass macromolecules and to do so at lower peptide concentrations than are required for wildtype melittin. ${ }^{39}$ Molecular dynamics simulations of the MelP5 mutant have suggested that this gain of function variant works in a similar fashion to the wild-type peptide. ${ }^{40}$ MelP5induced membrane pores have been studied by electrochemical impedance spectroscopy, macromolecule leakage assays, and single ion channel recording, but the dynamic topographic structure of MelP5-induced membrane remodeling remains uncharacterized..$^{33,39}$

Atomic force microscopy (AFM) is a powerful single molecule tool that provides a real-time real-space view of membrane topography with potentially sub-nm lateral resolution. ${ }^{41-42}$ This method allows for direct imaging of peptide-induced remodeling of a fluid lipid bilayer in near-native conditions, and has been used to observe defects induced by wildtype melittin. ${ }^{16}$ This capability opens the door for studies of nuanced effects, such as the colocalization of the thinning of the bilayer and pores, which can go undetected by other methods. 
Here we employed AFM imaging to study the interaction of MelP5 with supported POPC lipid bilayers. MelP5 created stable, highly localized pore-like voids in the membrane with diameters out to around $80 \AA$. Bilayer remodeling was observed in two distinct modes: shallow non-localized thinning, or deep highly localized voids. MelP5 exchanged between these two states reversibly in an apparent dynamic equilibrium. Further, the two states were found to be colocalized.

\section{Results and Discussion}

\section{Overview of atomic force microscope imaging}

We studied the topographical consequences of MelP5 binding to lipid bilayers. To this end, we performed tapping mode AFM imaging of supported bilayers made from liposomes which had been incubated with peptides prior to fusion onto mica supporting surfaces. ${ }^{43}$ 1-palmitoyl-2-oleoyl-sn-glycero-3-phosphocholine (POPC) was chosen as a model bilayer due to its prevalence in many eukaryotic cellular membranes. ${ }^{10,44-45}$

Previous studies have shown that MelP5 affects lipid bilayers at a variety of concentrations, including significantly lower than those required for wild-type. ${ }^{39} \mathrm{AFM}$ imaging trials were conducted at varying concentrations of MelP5. Conditions were optimized to yield a significant number of topographic features while simultaneously avoiding large-scale destruction of the membrane. Experiments at high peptide-to-lipid ratios $(\mathrm{P}: \mathrm{L}=1: 240$ or 1:130) led to widespread membrane distortion, whereas trials at lower concentrations led to too few features for statistical analysis. It was found that P:L $=1: 1240$ was optimal for our study, which focused on characterizing the topography of MelP5-induced membrane remodeling. 

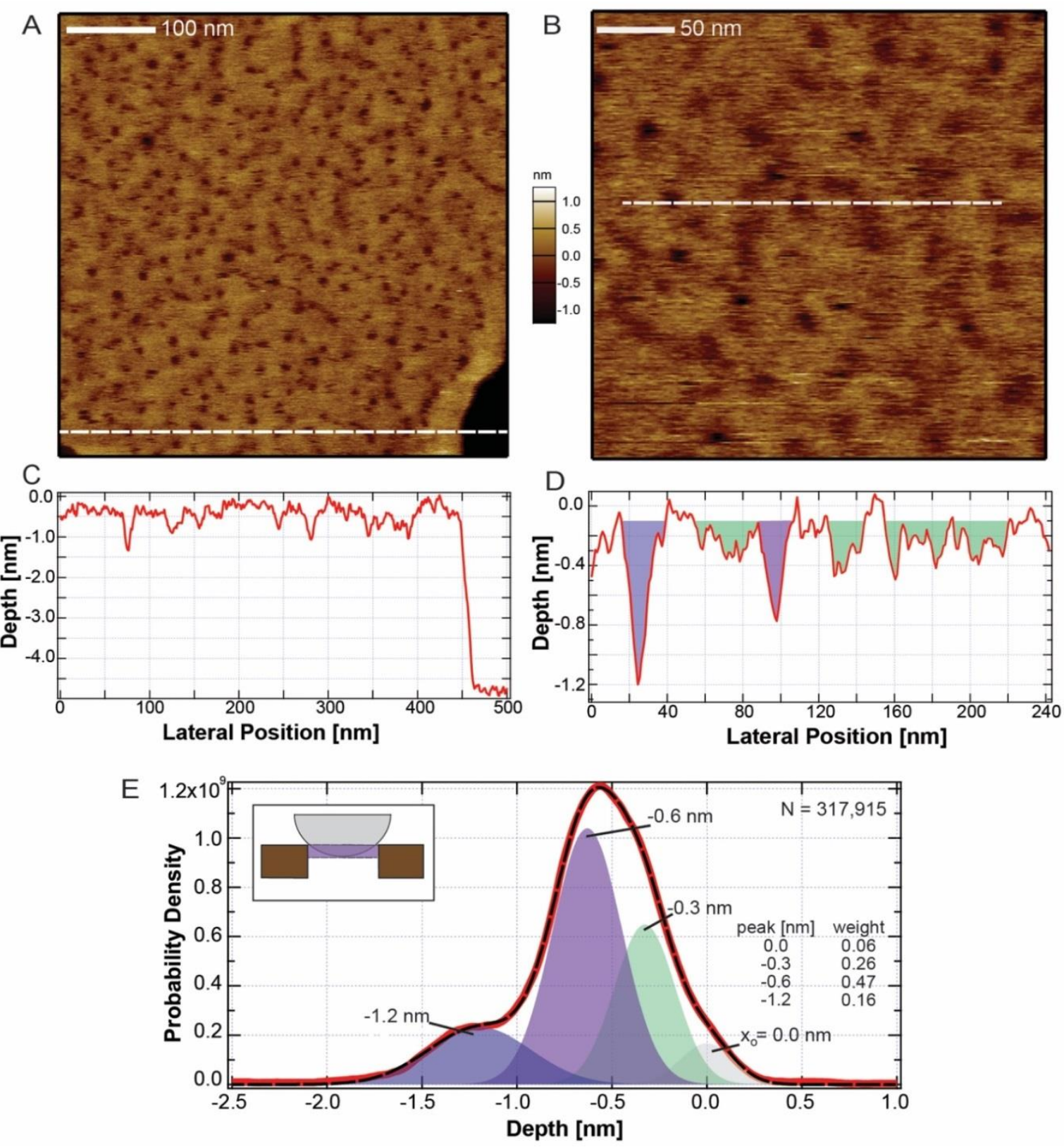

Figure 13 (A, B): Representative images of the membrane affected by MelP5. (C) Line scan from image in (A), position indicated by dashed line; note the characteristic $4 \mathrm{~nm}$ thickness of the bilayer. (D) Line scan taken from image in (B), with certain topographic populations highlighted. (E) Histogram compiled of pixels from representative images taken from three independent experiments. Data is shown (red), the sum fit (black dashed). E, Inset: Cartoon showing an explanation for a shallow reading.

Figure $13 \mathrm{~A}$ shows a representative image $\left(500 \times 500 \mathrm{~nm}^{2}, \mathrm{P}: \mathrm{L}=1: 1200\right)$.

Numerous punctate depressions in the membrane surface are visible. A line scan through 
the image (Figure 13C, white dashed in Figure 13A) reveals the depth of several depressions and verifies the presence of the lipid bilayer via its characteristic $40 \AA$ thickness. ${ }^{46}$ As a control, membrane samples prepared in an identical manner, but without MelP5, were shown to be essentially featureless. Figure 13B is an image of MelP5-treated membrane at increased magnification $\left(290 \times 290 \mathrm{~nm}^{2}\right)$. The associated line scan (Figure 13D) highlights two localized pore-like features (purple shades) as well as a diffuse region of thinned membrane (green). Note, the vertical scale in the images (Figure 13A,B) is distinct from the line scans (Figure 13C,D); zero depth in the line scans delineates the top surface in the images, which we take to be the surface of the unmodified upper leaflet of the bilayer. Clearly, MelP5 has a significant effect on membrane topography.

To quantify the distribution of topographic distortions further, a histogram from data acquired in the membrane region of the image (excluding the underlying mica surface) was compiled. This histogram of pixel weights (Figure 13E) reveals several important topographic populations. There appears to be one prominent state exhibiting a depth of $-0.3+/-0.2 \mathrm{~nm}($ mean $+/-\sigma)$ below the top of the bilayer, and two other states with depths of $-0.6+/-0.2 \mathrm{~nm}$, and $-1.2+/-0.3 \mathrm{~nm}$, respectively. These populations are robust; the histogram (Figure 13E) was compiled using data from three independent experiments.

In terms of relative weights, the $0.6 \mathrm{~nm}$ deep state was the most common topographical distortion observed at this $\mathrm{P}: \mathrm{L}=1: 1240$. It occurred with a probability per area approximately 3 -fold higher than the deeper $1.2 \mathrm{~nm}$ state. The $0.3 \mathrm{~nm}$ deep state of the membrane was found with an intermediate probability. To summarize, direct inspection of AFM images coupled with histogram analysis of image pixels identified 
distinct topographic states of MelP5-induced membrane distortion as well as relative weights.

\section{Characterizing pore-like voids in the bilayer}
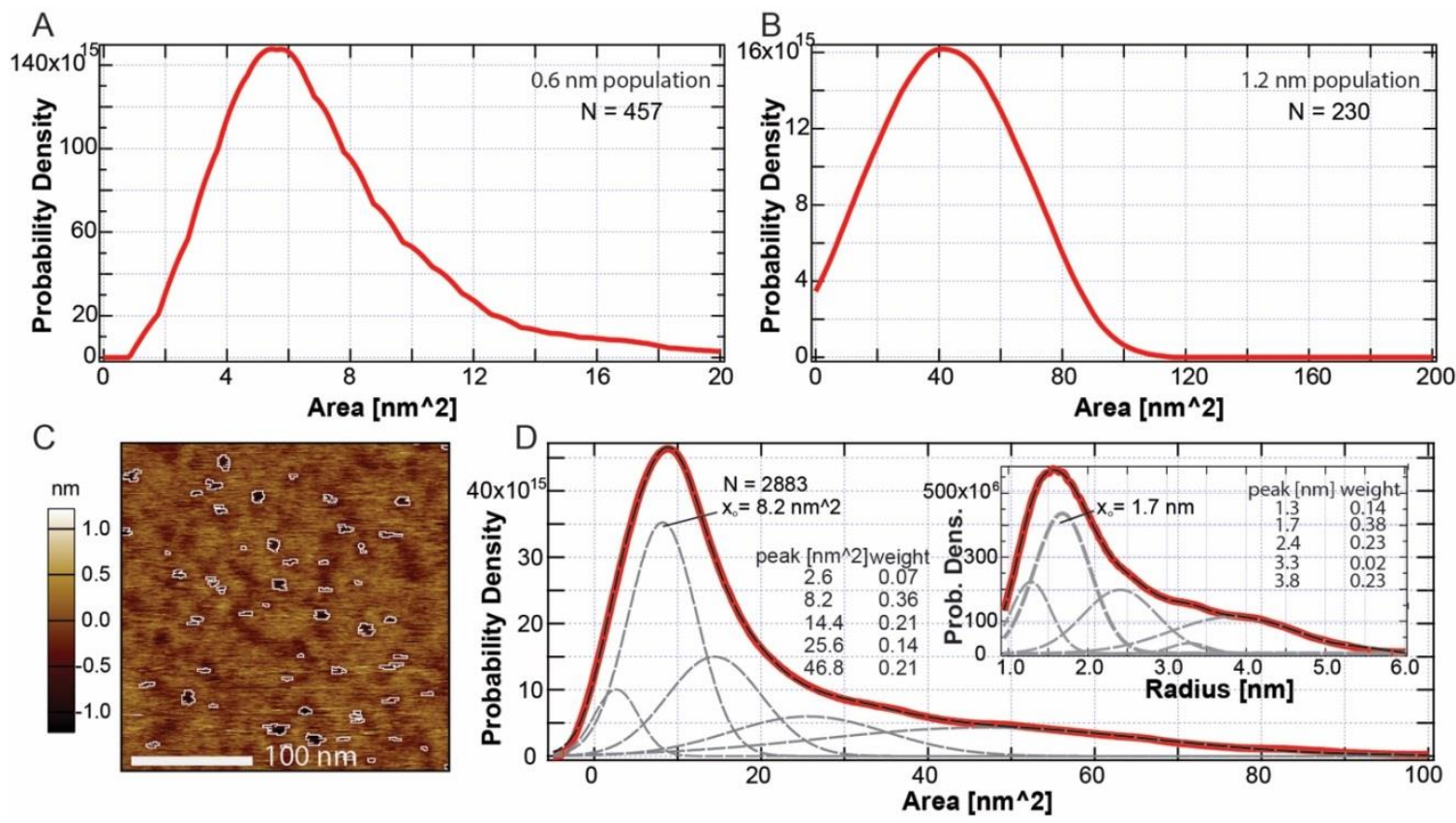

Figure 14. Geometric analysis of pores. Histograms of footprint areas constructed from subpopulations of voids exhibiting depths (A) $-0.6 \mathrm{~nm}(+/-0.1 \mathrm{~nm})$ or (B) $-1.2 \mathrm{~nm}(+/-0.1 \mathrm{~nm})$. In both cases, the distributions are sharply peaked, indicating that these voids are highly localized. (C) The program picked out exclusively the pores (white contours), and did not analyze the thinned portions of the membrane. (D) Histogram of pore footprint areas. The histogram has a major population at an area of $8.2 \mathrm{~nm} 2$ but has subpopulations that go out as far as $46.8 \mathrm{~nm} 2$. The weight of each peak is listed (grey dashed indicate the Gaussian fits). The data is shown in red, while the dashed black line is the sum fit. Inset: Histogram of pore radii.

To further elucidate the size and form of the voids, pore-like features were isolated algorithmically. Images were analyzed using custom software that located voids in a given image with depths greater than $0.3 \mathrm{~nm}$, controlled by the blob strength parameter in the Hessian blob algorithm. ${ }^{47}$ We explored the relationship between measured void depth and footprint area. 
By their nature, pores are highly localized voids within an otherwise planar bilayer surface. Both the shallow $(-0.6 \mathrm{~nm})$ and the deep $(-1.2 \mathrm{~nm})$ populations of voids exhibited sharply peaked footprint area histograms (Figure 14A \& B). If these two populations represented a deep mode of non-localized membrane thinning, then one would expect no underlying structure and no preferred area. The data indicated that both of these void populations were highly localized, and hence pore-like. Deeper voids exhibited larger areas. Due to their localized nature, different measured pore depths could arise from pores with different diameters. For example, when imaging pores of the same depth but with different diameters, the tip would read a shallower depth for the smaller diameter pore (Figure 13E, Inset). Due to sharpness and aspect ratio limitations of the AFM probe (Figure 1), the tip was not able to penetrate deeply into the MelP5 pores. However, the underlying structure of the $0.6 \mathrm{~nm}$ and $1.2 \mathrm{~nm}$ deep features suggests that these two populations are indeed pores.

Once identified (Figure 14C, white contours), a histogram of pore footprint areas was compiled (Figure 14D). Assuming a circular geometry, which matched many of these features, a histogram of radii was also created (Figure 14D, Inset). As seen in Figure 4D, the two most prominent populations of footprint area for MelP5 pores were $8.2+/-4.1$ $\mathrm{nm}^{2}$ and $14.4+/-5.7 \mathrm{~nm}^{2}$. These populations give rise to radii of $1.7+/-0.4 \mathrm{~nm}$ and 2.4 $+/-0.5 \mathrm{~nm}$, respectively. This geometry is in overall agreement with previous results, which put the radius of MelP5 pores at $\geq 2 \mathrm{~nm}$, due to its release of $10 \mathrm{kDa}$ dextran. ${ }^{39}$ Though these two populations together constituted more than half of all the pore-like features observed, a significant population (23\% of total) exhibited a larger radius of $3.8+/-0.8 \mathrm{~nm}$. Given the challenges in measuring longitudinal pore geometry 
via $\mathrm{AFM},{ }^{48}$ the radii and footprint areas reported here are taken to be an upper limit on the narrowest constriction of the pore. Thus, MelP5 does not create one size of pore, but rather has a variability of pore footprint areas. This observation, which is based on direct imaging of bilayer topography, is consistent with recent electrophysiology results, ${ }^{33}$ though Fennouri et. al employed bilayers from mixed lipid species and here we used POPC.

\section{Characterizing thinned regions of the bilayer}

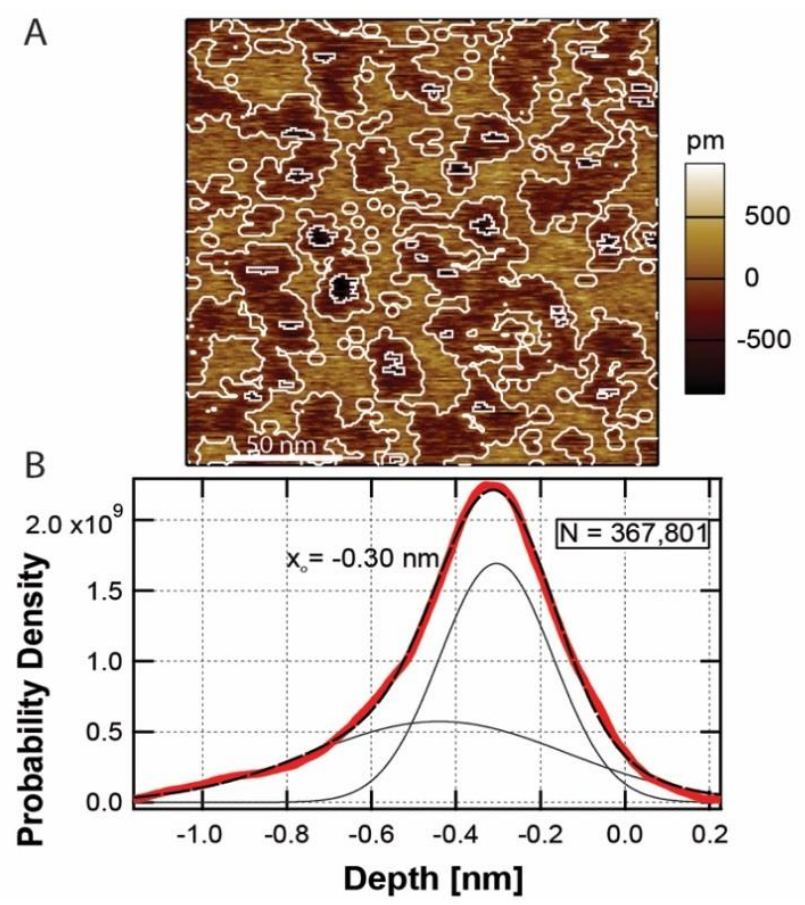

Figure 15 Analysis of thinned regions of membrane. (A) Thinned regions were identified algorithmically and differentiated from taller regions of the bilayer and deeper pore-like structures. (B) Histogram of the thinned region exhibiting a peak at $-0.30+/-0.14 \mathrm{~nm}$.

Spatially inhomogeneous and de-localized thinned regions of the membrane were frequently observed in the AFM images. The thinned areas of the membrane had shallower depths and dynamic, less definite structure as compared to the highly localized pores. Thinned areas of the membrane were selected algorithmically in order to more precisely define their structure. Then, a histogram was compiled using only the affected 
segments of the membrane. As seen in Figure 15, the most probable depth of the thinned area was $-0.30+/-0.14 \mathrm{~nm}$. This agrees with and refines the $-0.3+/-0.2 \mathrm{~nm}$ peak identified in Figure 13. Tip restricted depth readings were less of a concern here, since the thinned portions were not deep and exhibited significantly larger areas as compared to both the pores and the AFM tip. However, we note that while thinned regions with small areas were rare (see Figure 16), they could not be differentiated from pores, given the limitations imposed by tip geometry.

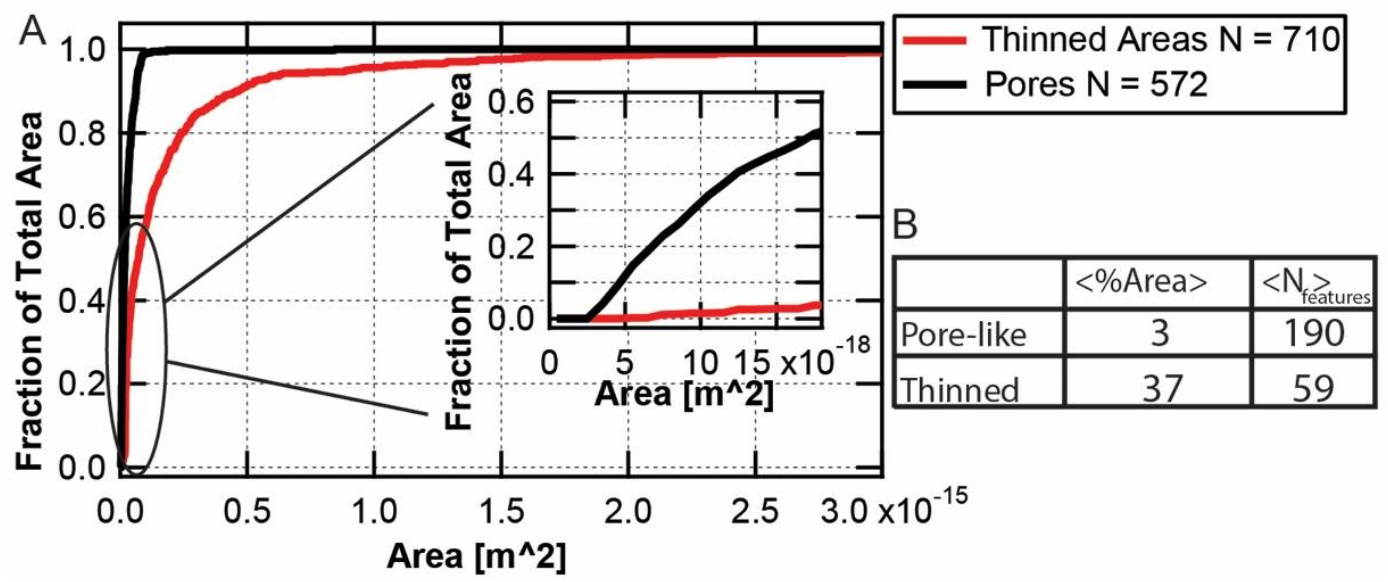

Figure 16. (A) Integrated area histograms for both the pore-like features (black) and the thinned features (red). Inset: A detailed view highlights the difference between the two populations. (B) Table comparing the pores and thinned features of the membrane. Two quantities are of interest: (i) the percent area of an average image taken up by the pores or the thinned features and (ii) the average total number of each feature type in an image.

The thinned features were characterized by their footprint area, to ascertain if they were indeed thinned only—exhibiting no underlying structure — or if they were poreswhich exhibit a high degree of localization. Analyzing the area histogram of the thinned features revealed a wide variety of possible footprint areas, the histogram for the thinned population has a shoulder much larger than the sharply peaked pore histogram. This 
difference in underlying structure is seen in Figure 16, where the integrals of the two area histograms are plotted. For the pores, the integral reaches unity very quickly-indicating that the vast majority of footprint areas are localized in a distinct peak. However, for the thinned areas, the integral does not saturate nearly as rapidly, owing to a lack of underlying structure.

For each image there was on average 59 thinned features that accounted for $37 \%$ of the total area of the image and 190 pores that accounted for only $3 \%$ of the total area of the image (Figure 16B). The membrane-thinned features were therefore much larger in area than the pore-like features.

\section{Colocalization of thinned membrane regions and pores}
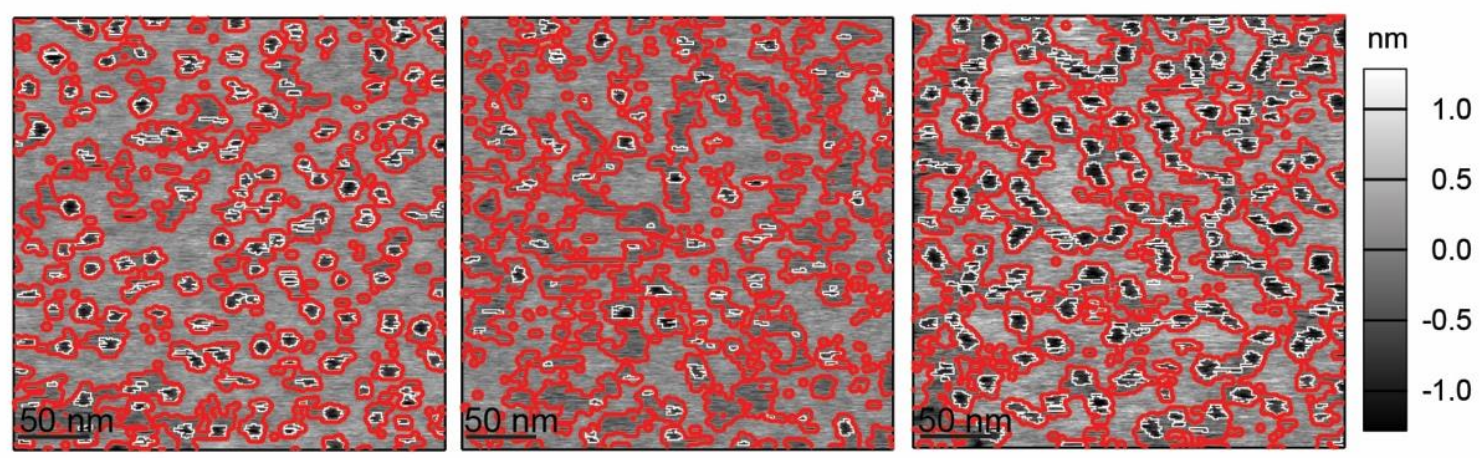

Figure 17. Colocalization of pores and membrane-thinned regions. Images of bilayers with MelP5 show that the pores-circled in white-fall within the flood mask (red) used to delineate the thinned areas of the membrane.

When analyzing the spatial distribution of the pores and the thinned portions of the membrane, we discovered that pores were always surrounded by or immediately adjacent to thinned areas of the membrane. Figure 17 shows representative images from three separate preparations highlighting this observation. Here the pores are circled in white and the thinned areas in red. In a popular model of pore formation, ${ }^{38}$ pores form in regions where enough peptides have already congregated and thinned the membrane; this 
membrane-bound pool of peptides can then act in a cooperative manner to form a pore.

This process has been simulated for melittin, but has not been experimentally observed. ${ }^{49}$

We emphasize that the observation of colocalization between membrane thinned regions and pores requires a single molecule approach. Using the AFM we were able to directly image the colocalization and thus shed light on the process of cooperativity. Previous studies have shown that wild-type melittin can thin the bilayer, ${ }^{31}$ but the lateral distribution and location of the thinning, and how it relates to pore location remained unknown. We observed that MelP5 causes laterally disperse and inhomogeneous thinning of the bilayer, and that these thinned regions have within or abutting them punctate pores.

\section{Topographical dynamics: transitions between membrane-thinned states and pore}

\section{states}
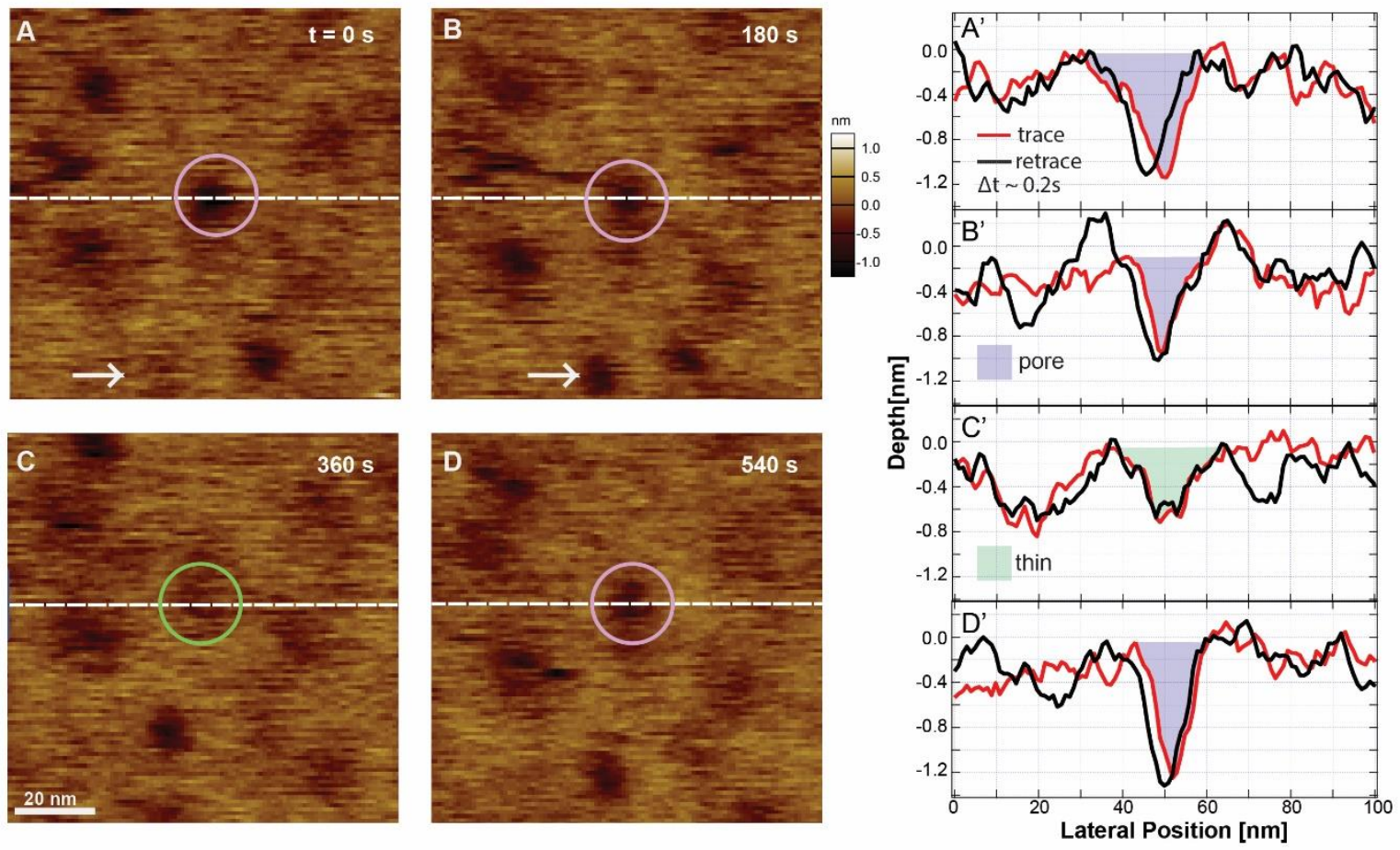

Figure 18. Dynamic transitions of MelP5-induced membrane topography. (A-D) Time lapse images of membrane, arrows and circles highlight interesting features. Line scans from each 
image ( $A^{\prime}-D^{\prime}$, line scan $A^{\prime}$ corresponds to image $A$, etc.) show a detailed view of a transition between pore states $(A, B, D)$ and thinned state $(C)$.

Continuously imaging the same area of the lipid bilayer surface gave us insight into the complex dynamics that underlie the peptide-lipid interactions. By direct inspection of AFM images we found that the peptides were able to exchange reversibly between the spatially diffuse membrane-thinned state and the highly localized pore state. For example, in Figure 18A \& B, the white arrow points to a section of the membrane where the topology changed from a thinned state (panel A) to a pore state (panel B). This change was registered by the membrane going from a shallow depth reading to a deeper depth reading (indicated by the change in color). The circles in Figure 18A-D highlight a region of the bilayer surface that shows that this process is reversible. The circled feature changes its topography from a pore state (panels A \& B) to a thinned state (panel C) and then back to a pore state (D). To evaluate this behavior more quantitatively, line scans (Figure 18A'-D', image panel A corresponds to line scan A', etc.) show how the feature's depth changes as a function of time. The elapsed time between trace and retrace in the AFM raster scan (red and black in Figure 18A'-D') was $\leq 200 \mathrm{~ms}$. We note that the circled feature in Figure $18 \mathrm{C} \& \mathrm{C}^{\prime}$ is labeled thin for two reasons: $(i)$ its depth falls within one standard deviation of the membrane thinned peak and (ii) topographically, it is not highly localized. More generally, this feature could represent a transitional state between the pore state and the thinned state of the membrane.

The time resolution of our AFM (one complete image every 180 seconds) sets an upper limit to the timescale of the observed topographic state changes. Analysis of fifty individual transitions similar to that circled in Figure 18 revealed that topographic transitions were not observed when examining sequential trace and retrace line scans over 
nominally the same area of the membrane. This is consistent with the data shown (Figure 18A'-D') and puts an approximate lower limit of 0.2 seconds on the time scale to transition between the thinned state and the pore state.

\section{Conclusions}

We provide novel characterization of membrane remodeling induced by the synthetically evolved gain of function peptide MelP5. Direct observations of lipid bilayer remodeling show that MelP5 interacts with the membrane and causes one of two general outcomes that are in equilibrium: delocalized membrane thinning or highly localized pores. We measured the thinning of the bilayer to be approximately $3 \AA$ a below the upper leaflet of the bilayer, in overall agreement with previous studies of wild-type melittin. When analyzing pores formed by MelP5, the most probable footprint area was found to be $8.2 \mathrm{~nm}^{2}$, which gives a most probable radius of $1.7 \mathrm{~nm}$. However, the area histogram exhibited a sizeable shoulder, thus stable pores (on the timescale of image acquisition) were observed with much larger footprint areas, out to about $47 \mathrm{~nm}^{2}$. Additionally, we have shown that the peptides exchanged reversibly between the thinned state and the pore state, and have suggested upper and lower bounds on the characteristic time scale of this transition $(0.2<\tau<180 \mathrm{~s})$. At the $\mathrm{P}$ :L ratio we utilized (1:1240) pores were always found surrounded by or abutting thinned areas of the membrane. This is consistent with cooperative pore-formation where the local concentration of peptides determines the state — inserted or membrane bound — of the peptide. ${ }^{38}$ When the local concentration reaches a threshold, a transmembrane pore is created. Under that threshold, the peptides only thin the membrane. In the future it would be interesting to vary the peptide concentration to explore this local thresholding phenomena further. In conclusion, MelP5 
has a profound influence on POPC bilayers and AFM imaging provides a direct visualization of the topographic changes imparted by the peptide in a physiologically relevant setting.

\section{Materials and Methods}

Peptides. MelP5 was synthesized (BioSynthesis Inc.) with a free N-terminus and Cterminal amidation. Identity and purity were verified with HPLC and mass spectrometry. The sequence of MelP5 is GIGAVLKVLATGLPALISWIKAAQQL. Prior to AFM study, the peptide was diluted to a final concentration of $1 \mu \mathrm{M}$ in imaging buffer: 100 $\mathrm{mM} \mathrm{NaCl}, 50 \mathrm{mM}$ TrisCl at $\mathrm{pH}$ 7.4.

Peptide-lipid bilayer preparation. We adopted established methods to form supported lipid bilayers on mica. ${ }^{50}$ Briefly, POPC (Avanti Polar Lipids) was suspended in $150 \mathrm{mM}$ $\mathrm{NaCl}, 40 \mathrm{mM} \mathrm{CaCl} 2$, and $20 \mathrm{mM}$ Hepes with a pH of 7.5 at a concentration of $62 \mathrm{mM}$. Liposomes were prepared by extrusion of POPC through a polycarbonate membrane (approximately 25 times) with a $100 \mathrm{~nm}$ pore diameter. POPC liposomes were incubated for 10 minutes with MelP5 at $25{ }^{\circ} \mathrm{C}$ to yield a final peptide concentration of $0.33 \mu \mathrm{M}$. The liposomes containing peptides were then deposited onto freshly cleaved mica.

Supported bilayers were formed by vesicle fusion $\left(30\right.$ minute incubation, $\left.\sim 30{ }^{\circ} \mathrm{C}\right) .{ }^{50}$ Prior to imaging, samples were rinsed ( $75 \mu \mathrm{L}, 4$ times) with imaging buffer. All experiments were performed at a peptide-to-lipid ratio of 1:1240 and at $\sim 30^{\circ} \mathrm{C}$, significantly above the gel-to-fluid transition temperature of POPC.

AFM imaging and analysis. Images were acquired in tapping mode in aqueous buffer solution using a commercial apparatus (Asylum Research, Cypher). Care was taken to control the magnitude of the tip sample force to $\leq 100 \mathrm{pN}$ (estimated by comparing the 
free amplitude to the set point amplitude). As is typical, images were flattened ( $2^{\text {nd }}$ order) to minimize background. When histograms were compiled, the data was offset so as to align the population having the greatest height (which we assumed to be the unaltered upper surface of the lipid bilayer) to zero. Individual pores were selected using custom software (Igor Pro 7, WaveMetrics), which gave details on the depth and footprint area of each pore ${ }^{47}$. Histograms were fit with multiple Gaussians (MagicPlot), with $\mathrm{x}_{0}$ defined as the center of the peak. 


\title{
Project Two: Macrolittin70
}

\author{
Potent Macromolecule-Sized Poration of Lipid Bilayers by the Macrolittins, A Synthetically \\ Evolved Family of Pore-Forming Peptides \\ Sijia Li, Sarah Y. Kim, Anna E. Pittman, Gavin M. King, William C. Wimley, and Kalina Hristova \\ Journal of the American Chemical Society 2018140 (20), 6441-6447
}

\section{Introduction}

Several thousand peptides that interact with and permeabilize lipid bilayer membranes have been described in the literature ${ }^{51}$. Peptides of this class have many potential uses in biotechnology, including use as antibacterial agents ${ }^{52}$, anticancer agents $^{53}$, as antiviral agents ${ }^{54}$, drug delivery vehicles ${ }^{55}$, transfection reagents $^{56}$, exogenous ion channels ${ }^{57}$ and more. Successful applications have been limited, however, by several impediments. For example, some membrane permeabilizing peptides are active only at high concentration. Also, most pore forming peptides form small, transient pores in bilayers ${ }^{17,58-59}$. Despite very active research over the past few decades, our understanding of the sequence-structure-function relationships for membrane active peptides remains mostly descriptive, and not predictive. Thus, the current state of understanding does not readily enable rational design or improvement of membrane permeabilizing peptide activity.

In the absence of reliable predictive rules and algorithms for engineering the activity of membrane permeabilizing peptides, we have been using synthetic molecular evolution, i.e. iterative combinatorial library design and high-throughput screening, to discover new functionalities ${ }^{23,29,60-65}$. We began this effort with a first generation in 
which we screened a library based on the cytolytic bee venom peptide melittin, which forms transient pores ${ }^{17,59}$, for gain-of-function analogs that form equilibrium pores at much lower concentration than melittin. We later showed that the best of the selected gain-of-function variants, called MelP5, readily released a $10 \mathrm{kDa}$ dextran and other macromolecules from lipid vesicles at low concentration ${ }^{39}$. We then used MelP5 as a template for a second generation screen in which we selected for release of a $40 \mathrm{kDa}$ dextran ${ }^{23}$ at low concentration in a manner that is triggered only at $\mathrm{pH}<5.5$. Because MelP5 causes macromolecular poration in a pH-insensitive manner, the library from which these "pHD peptides" were selected included six protonatable aspartate or glutamate residues, among other variations, in a MelP5 template. The selected pHD peptides are inactive at $\mathrm{pH} 7$, but are triggered by low $\mathrm{pH}$ to cooperatively bind to membranes, fold into amphipathic $\alpha$-helices and induce membrane destabilization that enables the release of macromolecules at least as large as $40 \mathrm{kDa}$ dextrans ${ }^{23}$. The $\mathrm{pH}$ values for $50 \%$ leakage are $\sim 5.5$, and the $\mathrm{pHD}$ peptides cause macromolecular poration at peptide-to-lipid ratios (P:L) lower than 1:500 ${ }^{39}$. The pHD peptides were more potent than MelP5 at pH 5, suggesting that MelP5 could be further optimized as a macromolecular pore-former.

In this work we sought to increase the diversity of well characterized peptides which can induce macromolecular poration. Specifically, we sought to identify new gainof-function peptides that form macromolecule-sized pores in zwitterionic phosphatidylcholine bilayers with the same high potency as the pHD peptides, but which are active at neutral $\mathrm{pH}$. To accomplish this, we used the same MelP5-derived library from which the $\mathrm{pHD}$ peptides were selected, and performed a different high-throughput 
screen to select for peptides with the desired properties. As a result of this screen, we identified the "macrolittins", a novel family of peptides that induce macromolecular poration at neutral $\mathrm{pH}$ at remarkably low peptide to lipid ratios.

\section{Materials and Methods}

Peptides and lipids. The peptide library was synthesized by a split and recombine method and validated as described previously ${ }^{23,60-62}$. Single peptides were synthesized and purified by Biosynthesis Inc., and were verified by HPLC and mass spec. Lipids were purchased from Avanti Polar Lipids. TAMRA-biotin-dextran (TBD) was synthesized and purified as described previously ${ }^{23}$.

Vesicle Preparation. Two types of large unilamellar vesicles (LUV) containing different dyes (ANTS/DPX and TAMRA-biotin-dextran) were prepared. POPC lipids in chloroform were dried and then resuspended in a buffer with ANTS/DPX or TAMRAbiotin-dextran. TBD containing vesicles were frozen and thawed ten times. Both types of vesicles were extruded through $0.1 \mu \mathrm{m}$ Nuclepore polycarbonate filters ten times to achieve a unilamellar uniform size. Vesicles with entrapped compounds were separated from unentrapped compounds by gel filtration chromatography for vesicles with ANTS/DPX, or by incubation on streptavidin agarose for vesicles with TBD. Lipid concentration was measured by Stewart Assay ${ }^{66}$.

Design of the Peptide Library. Previously, we described the design of the library from which we selected $\mathrm{pH}$-sensitive macromolecular pore formers ${ }^{23}$. In short, we used the sequence of $\mathrm{MelP5}^{39}$ as a template, and allowed six position to be either the native residue, protonatable aspartate or glutamate, or sometimes an additional nonpolar residue. 
Positions 7 and 21, which are lysine in MelP5, were allowed to be either lysine or histidine. The isoleucine at position 17 was also allowed to be glutamine. The acidic residues are arranged to lie on one face of the amphipathic helix.

High-throughput screen. The library was synthesized as a one bead one sequence library and was screened using a two-assay orthogonal screen. First, $\sim 0.5 \mathrm{nmol}$ of each library member in solution was assayed for induction of leakage of the small molecules ANTS and $\mathrm{DPX}^{67}$ from POPC vesicles at $\mathrm{pH} 7$ and a peptide to lipid ratio $(\mathrm{P}: \mathrm{L})=1: 400$. TBD vesicles had also been added to screen for TAMRA-biotin-dextran (40 kD) leakage at pH 7 and P:L = 1:400. For controls we also tested 1 and 4 nmol of MelP5 for partial and near complete macromolecule leakage, respectively. We also tested no peptide for a negative control and Triton-X100 detergent for a positive control for complete leakage. After selection of the positive pore forming library members (see text) the peptide remaining on the bead was sequenced by Edman degradation.

Tryptophan Fluorescence. A Fluorolog3-22 spectrofluorometer with double-grating excitation and emission monochrometers was used to measure fluorescence spectra. The emission polarizer was oriented 0 degree relative to the vertical and the excitation polarizer at 90 degrees. An excitation wavelength of $280 \mathrm{~nm}$ was used. Emission spectra were collected in the range of $300 \mathrm{~nm}$ to $450 \mathrm{~nm}$ for each sample.

Circular Dichroism (CD). CD spectra of MelP5 and macrolittins were measured in pH 7 phosphate buffer, and pH 5 acetate buffer, with and without $1 \mathrm{mM}$ POPC vesicles. All CD spectra were recorded on a Jasco J715 CD spectrometer. Wavelength scans from 280 
to $190 \mathrm{~nm}$ were performed in a $0.1 \mathrm{~cm}$ path length quartz cuvette using a slit width of 1 $\mathrm{nm}$ and a scan rate of $20 \mathrm{~nm} / \mathrm{min}$.

Oriented Circular Dichroism. Mixtures of peptide and lipid at P:L = 1:50 were prepared in methanol. Aliquots were dried onto a quartz disk which was sealed in a chamber with a second quartz window. The samples were hydrated through the vapor phase using a drop of distilled water to the chamber to form stacked oriented multibilayers. The quartz disk was oriented perpendicular to the beam and CD spectra were collected at eight rotations of the sample holder around the beam axis and averaged.

Sample preparation for atomic force microscopy $(A F M)$ imaging. We adopted established methods to form supported lipid bilayers on mica surfaces ${ }^{42}$. Briefly, POPC was suspended in $150 \mathrm{mM} \mathrm{NaCl}, 40 \mathrm{mM} \mathrm{CaCl}_{2}$, and $20 \mathrm{mM}$ HEPES, pH 5, at a concentration of $65 \mu \mathrm{M}$. Liposomes were prepared by extrusion of POPC through a polycarbonate membrane (approximately 25 times) with a $100 \mathrm{~nm}$ pore diameter. The peptide was diluted to a final concentration of $5 \mu \mathrm{M}$ in imaging buffer: $100 \mathrm{mM} \mathrm{NaCl}, 50$ mM HEPES at pH 5. POPC liposomes were incubated for 15 minutes in solution with the peptide to yield a final peptide concentration of $2.5 \mu \mathrm{M}$. The liposomes containing peptides were then deposited onto freshly cleaved mica. Supported bilayers were formed by vesicle fusion (30 minute incubation, $\sim 30^{\circ} \mathrm{C}$ ). Samples were rinsed four times using $75 \mu \mathrm{L}$ of the imaging buffer. All data was collected at $\sim 32{ }^{\circ} \mathrm{C}$, which is significantly above the gel-to-fluid transition temperature of POPC.

AFM imaging and analysis. Images were acquired in tapping mode in aqueous buffer solution using a commercial apparatus (Asylum Research Inc., Cypher) and Biolever 
mini tips with spring constant $k \sim 0.9 \mathrm{~N} / \mathrm{m}$ (Olympus, BL-AC40TS). Care was taken to control the magnitude of the tip-sample force to $\leq 100 \mathrm{pN}$ (estimated by comparing the free amplitude to the set point amplitude). As is typical, images were flattened (2nd order) to minimize background. For statistical analysis, the data was offset so as to align the population having the greatest height, corresponding to the top surface in each image, to zero. Defects were identified using a flood mask set at $-0.3 \mathrm{~nm}$ with respect to this top surface.
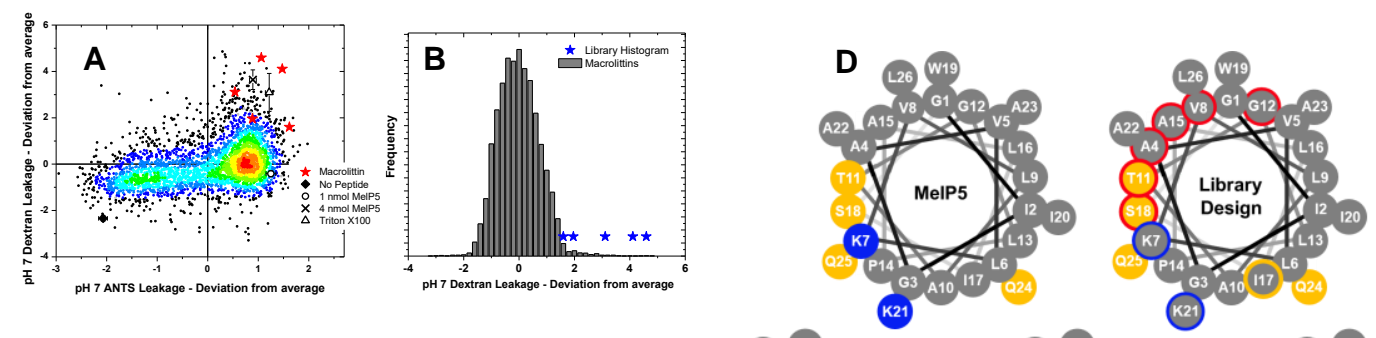

C

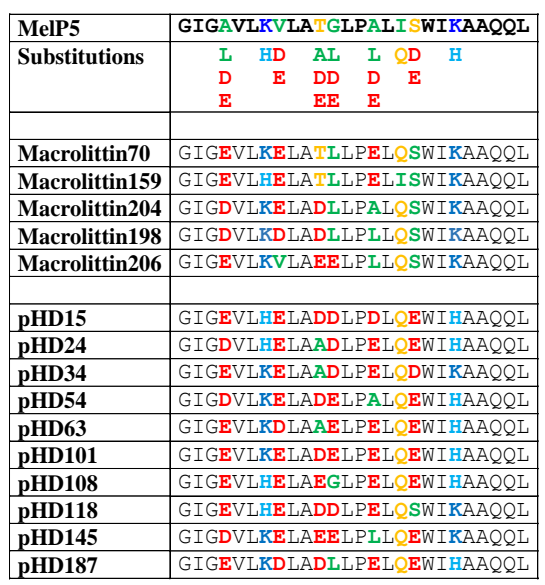
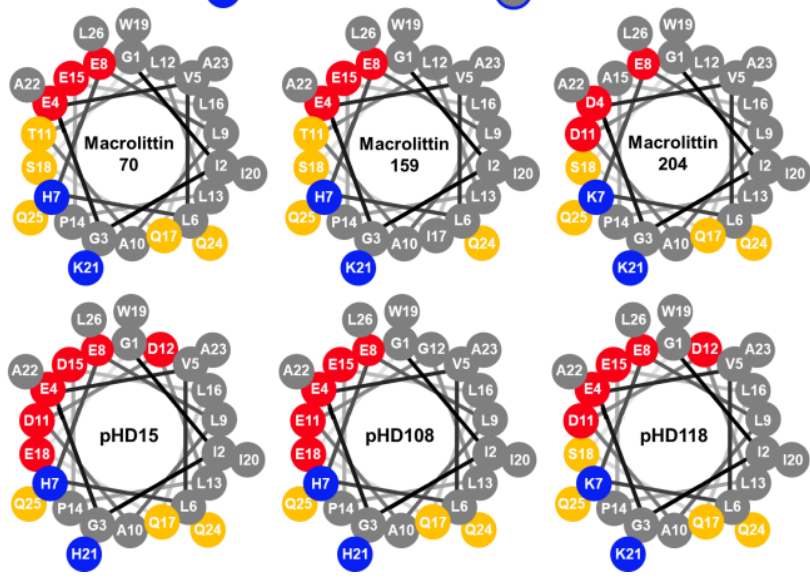

Figure 19 The selection of macrolittins by synthetic molecular evolution. A. The results of the screen. Z-values (library member-plate mean)/ (plate standard deviation) for each library member are shown for the small molecule screen at $\mathrm{pH} 7$ and for the macromolecule screen at pH 7. The macrolittins, red stars, were selected for simultaneous potent pore-forming activity in both screens. B. Histogram of macromolecule leakage values for the library members screened at $\mathrm{P}: \mathrm{L}=1: 400$ and $\mathrm{pH}$ 7. The macrolittins are in the most active $1 \%$ of the peptides 
screened. C. Table of peptide sequences. The sequence of the parent MelP5 and the MeIP5 derived library is shown. The macrolittin sequences and pHD peptide sequences selected during the screens are also shown. Bold residues were varied. Acidic residues are red, basic residues are blue, hydrophobic residues are green and polar residues are orange. D. Helical Wheel projections of the peptides discussed in this work. Acidic (red), basic (blue) and polar (yellow) residues are shown to highlight amphipathicity. Varied residues in the library are indicated by colored outlines, where red means acidic residues were included and blue means lysine and histidine were possible.

\section{Results}

\section{Macromolecule Leakage Screen}

The library design, shown in Figure 19, uses MelP5 as a template and includes six sites in which protonatable aspartate or glutamate residues, as well as the original residue, were possible. In the screen, modified from one described previously ${ }^{23}$, two assays were performed in series. First, leakage of the small molecule fluorophore ANTS and its quencher DPX, was measured at pH 7 and at P:L of 1:400. Next, macromolecular leakage was measured on vesicles containing entrapped TAMRA-biotin-dextran 40kD (TBD) and external AlexaFluor488-steptavidin at a P:L to 1:400. Leakage of TBD was measured by assessing FRET between TBD and the labelled streptavidin after 1 hour of incubation.

We screened 3200 library members, and the distribution of Z-values (standard deviations from individual plate averages) for the two assays are shown in Figure 19A. Controls included no peptide, Triton-X100 detergent for $100 \%$ release, and two amounts of MelP5; 1 nmol which causes partial macromolecule release and 4 nmol which causes near total macromolecule release. For comparison, there was about $0.5 \mathrm{nmol}$ of each 
library member present in the screen. The MelP5 controls correspond to P:L of 1:200 and 1:50, respectively. Both concentrations of MelP5 release nearly $100 \%$ of the small molecules ANTS and DPX while only the higher concentration releases most of the dextran.

The majority of library members screened cause significant small molecule leakage, and some macromolecule leakage. Here, we are interested in the outliers that cause significant macromolecule release. Therefore, positive peptides were selected from the area around the upper right hand quadrant of the plot. The sequenced library members are shown as blue stars. This quadrant represents simultaneous high small molecule leakage at $\mathrm{pH} 7$ and high macromolecule leakage at $\mathrm{pH}$. While a significant portion of the library causes high ANTS/DPX leakage, the selected macrolittins are found in the upper 1 percentile of the screened sequences on the macromolecular leakage axis (Figure 19B). Thus, these peptides are gain-of-function daughter sequences of MelP5; they form macromolecule size pores in PC bilayers at $\mathrm{pH} 7$ and at low peptide concentration. The screen suggested that the selected positives are much more potent than the parent peptide MelP5 at macromolecular poration, because $\sim 0.5 \mathrm{nmol}$ of these library members in the screen wells released significantly more of the $40 \mathrm{kDa}$ dextran than the $1 \mathrm{nmol}$ MelP5 control, and as much as the 4 nmol MelP5 control.

\section{Sequence Analysis}

The sequences of five selected peptides, which we named "macrolittins" are shown in Figure 19C along with the sequences of MelP5, the MelP5-based library, and the pHD peptides, for comparison. In Figure 19D we show helical wheel representations 
of the idealized helical surfaces of the parent MelP5, and representative members of the sequence families. The macrolittins have a sequence motif that always contains three acidic residues, either glutamic or aspartic acid out of six possible acidic residues in the library. The probability of this occurring by chance five times (p) is 0.013 . The acidic residues at the fourth and eighth positions are highly conserved (9/10 in total) in the five macrolittins. They are also conserved in the ten pHD peptides (20/20 total). The macrolittins sometimes have acidic residues in position 11 (3/5 peptides), position 15 (2/5), infrequently in position $12(1 / 5)$ and position $18(0 / 5)$, averaging about 1.0 acidic residues per peptide in those four positions. In comparison, each $\mathrm{pHD}$ peptide has either 3 or 4 acidic residues in those four positions, averaging 3.2 acidic residues per sequence in those four positions. More specifically, while positions 12 and 18 are acidic in the pHD peptides in 17 out of 20 chances, they are rarely acidic in the macrolittins (1/10) giving a probability of $<0.0001$ for this to have arisen by chance.

For the basic residues in positions 8 and 21 of the macrolittins, lysine was selected over histidine, 9 times in 10 chances $(\mathrm{p}=0.01)$. This demonstrates a strong preference for lysine in the macrolittins that does not exist in the pHD peptides. Slightly more than half $(11 / 20)$ of the possible basic residues in the $\mathrm{pHD}$ peptides were histidine instead of lysine, indicating that there is no preference. We note that both histidine and lysine are charged at $\mathrm{pH}<5.5$ where the $\mathrm{pHD}$ peptides are active, while only lysine is charged at $\mathrm{pH} 7$ where the macrolittins are active. 

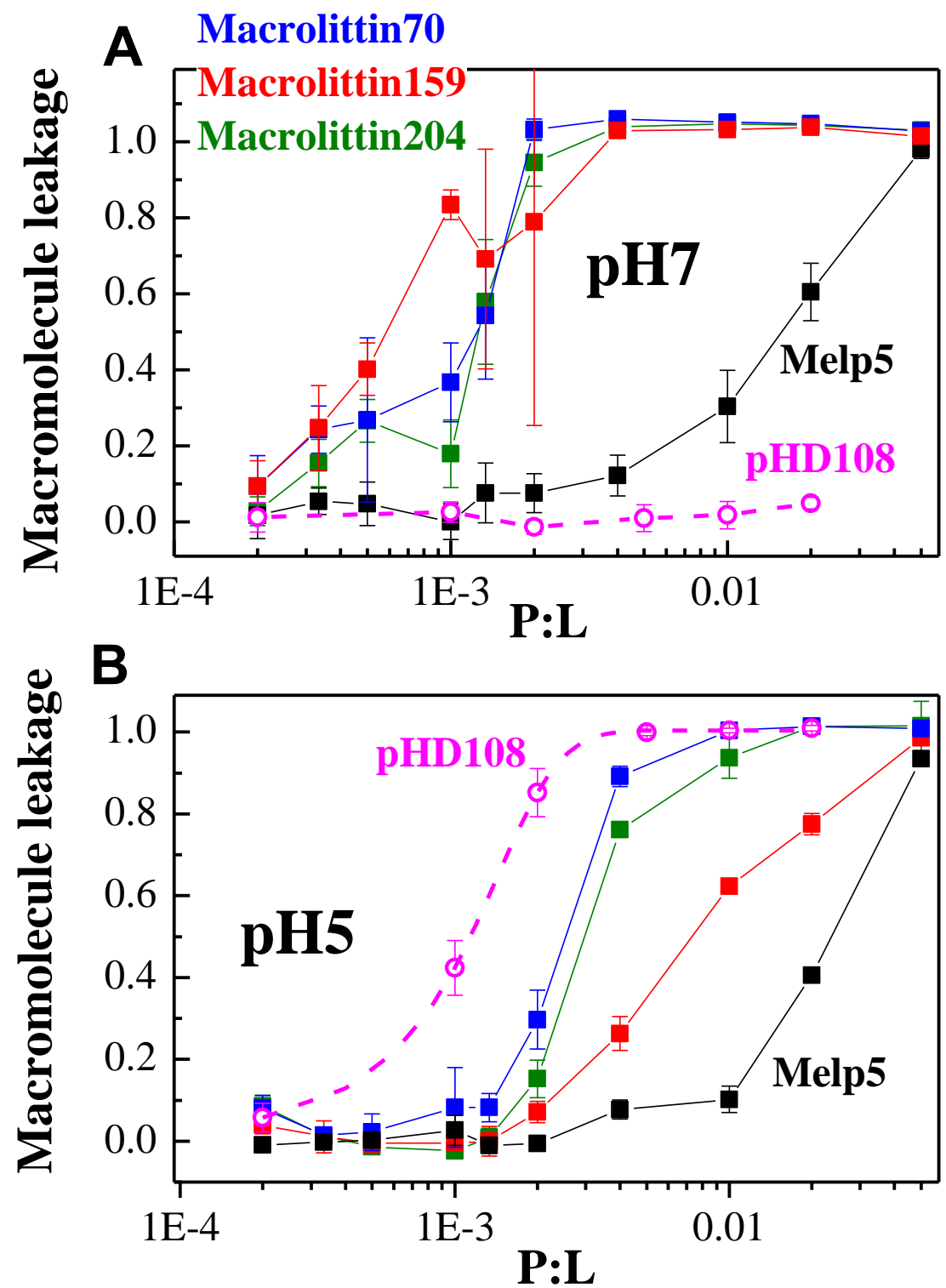

Figure 20 Macrolittin induced leakage of a $40 \mathrm{kDa}$ dextran from POPC vesicles. Vesicles with entrapped TAMRA-biotin-dextran $40 \mathrm{kDa}$ (TBD) were incubated with serial dilutions of peptide in the presence of Alexafluor488-streptavidin (AF488-SA) for 1 hour. FRET between TBD and AF488-SA was measured, followed by disruption with Triton-X100 to determine the FRET for complete leakage. A. Experiments at pH 7, where the pHD peptide pHD108 is inactive. B. Experiments at pH 5, where pHD108 is active.

Macromolecule Leakage 
To fully characterize the sequences identified in the screen, we synthesized three representative macrolittins, 70, 159 and 204 (Figure 19) and measured their ability to permeabilize lipid vesicles made from POPC to a $40 \mathrm{kDa}$ dextran as a function of peptide concentration. The leakage activity curves at $\mathrm{pH} 7$ are shown in Figure 20A. Macrolittins release TAMRA-biotin dextran (40 kDa) with a leakage inducing concentration of 50\% effect ( $\left.\mathrm{LIC}_{50}\right)$ of $\mathrm{P}: \mathrm{L} \leq 1: 800$. This is remarkably potent activity for macromolecule release, matched only by the pHD peptides at pH 5 (Fig. 2B). While MelP5 releases a 10 $\mathrm{kDa}$ dextran at similar $\mathrm{LIC}_{50}{ }^{39}$ its ability to release a $40 \mathrm{kDa}$ is much lower, with $\mathrm{LIC}_{50}$ at $\mathrm{P}: \mathrm{L}=1: 50$ (Fig. 2A). For comparison, the most active pHD peptides, at $\mathrm{pH} 5$, release 40 $\mathrm{kDa}$ dextran with $\mathrm{LIC}_{50}$ values of $\mathrm{P}: \mathrm{L}=1: 800^{23}$, similar to the macrolittins at $\mathrm{pH} 7$.

We also measured macrolittin-induced dextran leakage at pH 5 (Figure 20B) to directly compare the macrolittins to the pHD peptides under identical conditions. Surprisingly, the two families have opposite $\mathrm{pH}$ dependences. Unlike the $\mathrm{pHD}$ peptides which gain activity at $\mathrm{pH} 5$, the macrolittins are somewhat less potent at $\mathrm{pH} 5$ than they are at $\mathrm{pH} 7$, with $\mathrm{LIC}_{50}$ values between 1:150 and 1:400. However, even at $\mathrm{pH} 5$, the macrolittins are more potent than MelP5. The activity of MelP5 is similar at both $\mathrm{pH}$ values as reported previously ${ }^{23}$. 


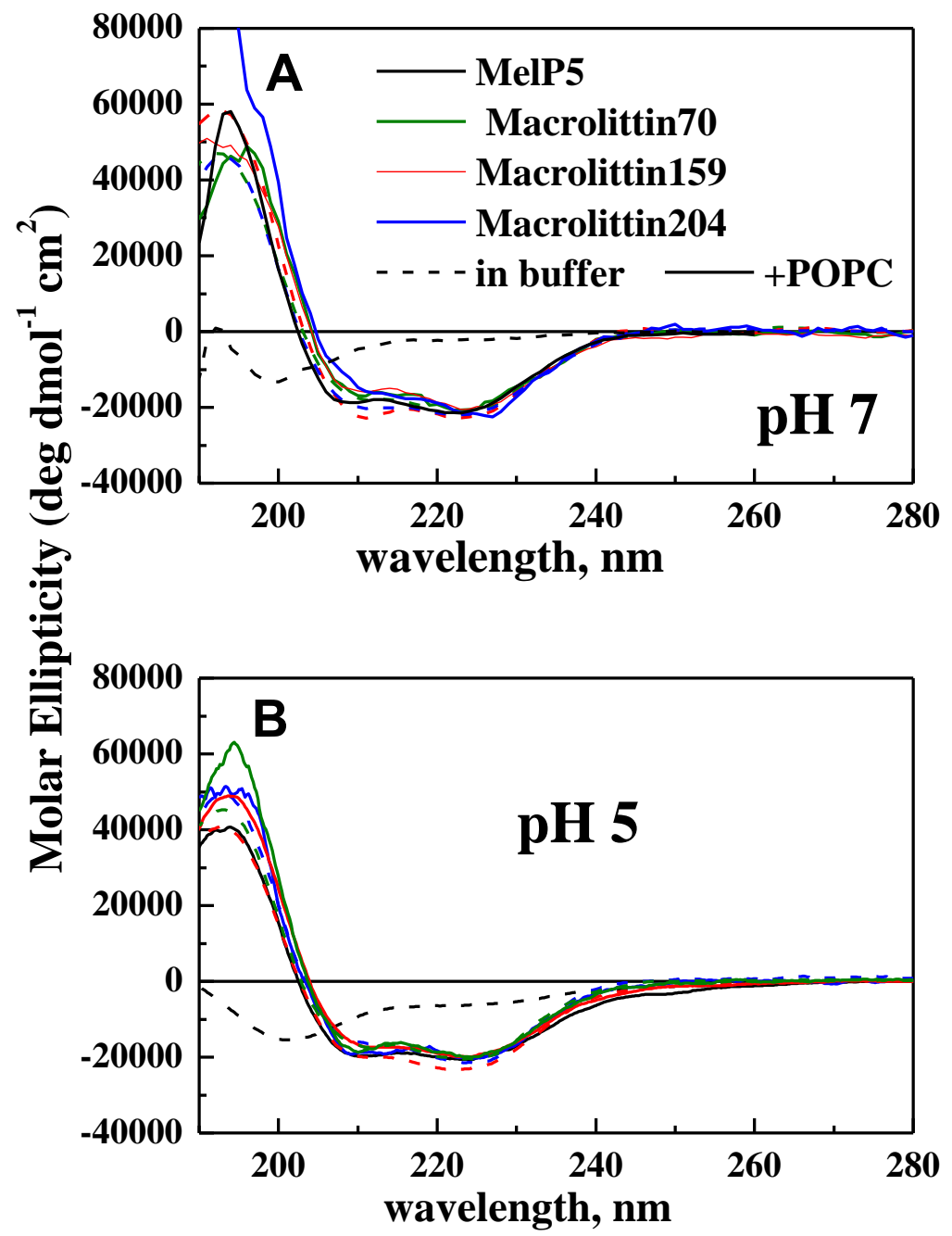

Figure 21 Solution circular dichroism spectra of macrolittins and MelP5 at 10 aM.

Measurements were made before addition (dashed lines) and after addition (solid lines) of 1 mM POPC vesicles. A. Measurements made at pH 7. B. Measurements made at pH 5.

Circular Dichroism and Tryptophan Fluorescence

To assess the secondary structure of the macrolittins, we measured their circular dichroism spectra, at $\mathrm{pH} 7$ and $\mathrm{pH} 5$, in buffer with and without lipid vesicles. The control peptide MelP5 is random coil in buffer and becomes highly helical only upon 
membrane binding (Figure 21). Surprisingly, the macrolittins are highly helical in buffer at pH 7 (Figure 21A), and also at pH 5 (Figure 21B), even in the absence of lipid vesicles. This unexpected finding suggests that they readily self-assemble into stable helical multimers in buffer perhaps like the melittin tetramers that form under some conditions ${ }^{68}$. In the presence of POPC vesicles, the macrolittins retain their wellorganized $\alpha$-helical secondary structure, while the control peptide MelP5 shows a sharp transition to $\alpha$-helix only upon binding to vesicles.

We used the fluorescence of the single tryptophan at position 19 of the peptides, to further assess their structure in buffer and in membranes. The tryptophan in MelP5, which is random coil and monomeric in buffer, has an emission maximum of $349 \mathrm{~nm}$ at $\mathrm{pH} 7$ and at $\mathrm{pH} 5$ as expected for a water-exposed tryptophan in a monomeric peptide (Figure 22). On the other hand, the macrolittins in buffer have tryptophan emission maxima from $319-333 \mathrm{~nm}$, significantly blue-shifted from those expected for waterexposed tryptophan. This observation is consistent with our conclusion that the macrolittins form helical multimers in buffer, as multimer formation would bury the Trp residue in a less polar environment. In the presence of POPC vesicles, MelP5 and the macrolittins have very similar tryptophan emission maxima of 320-330 nm, signifying a similar environment in the membrane. These emission maxima are in the expected range for a tryptophan that is membrane inserted ${ }^{69}$ as we have reported for MelP $5^{61}$ and the pHD peptides at $\mathrm{pH} 5$ in bilayers ${ }^{23}$. 

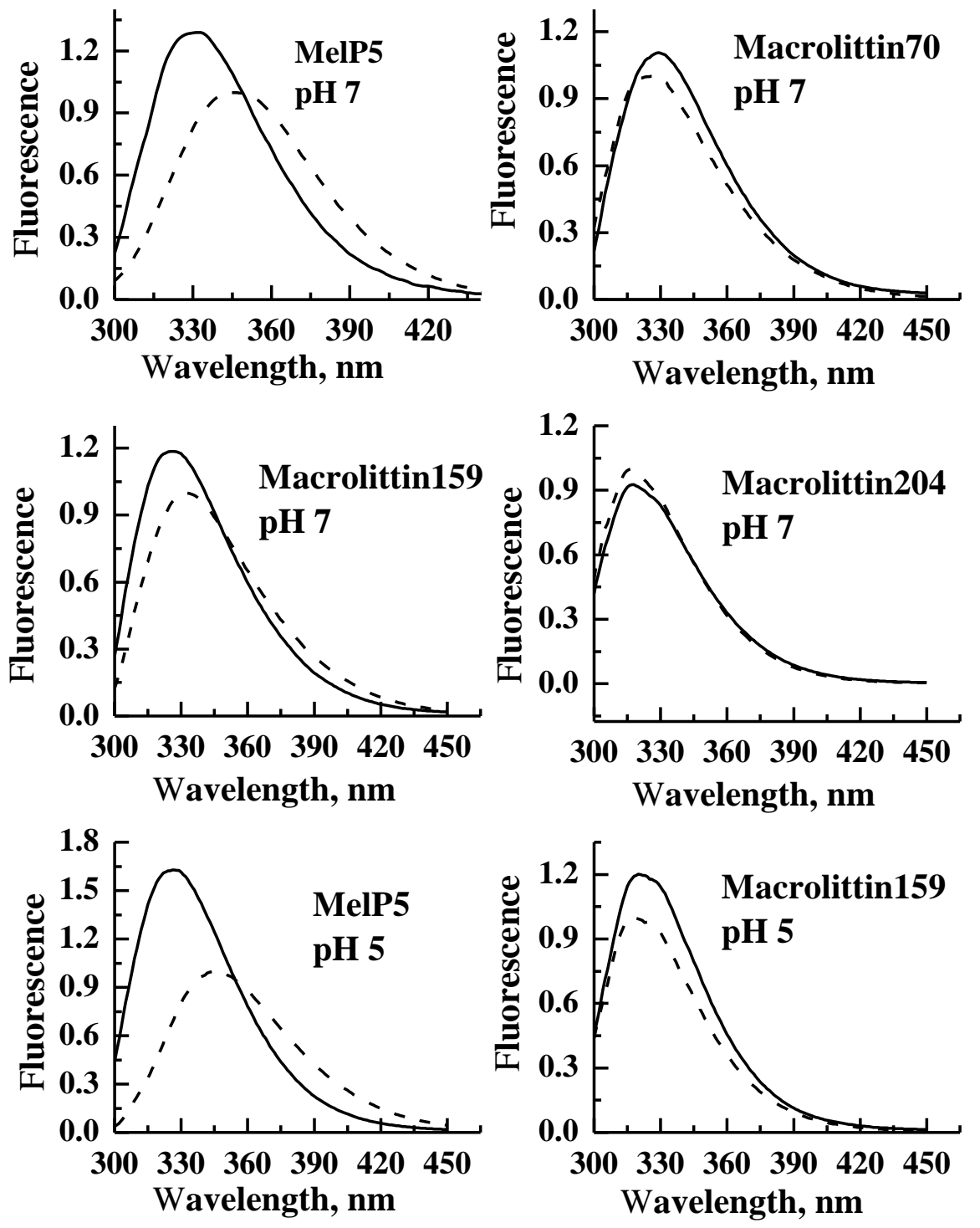

Figure 22 Tryptophan fluorescence spectra of the macrolittins and MelP5. Spectra were measured for 5 uM peptide before addition (dashed lines) and after addition (solid lines) of 1 mM POPC vesicles. Excitation was at $280 \mathrm{~nm}$. Emission spectra are normalized to the maximum intensity in buffer. 

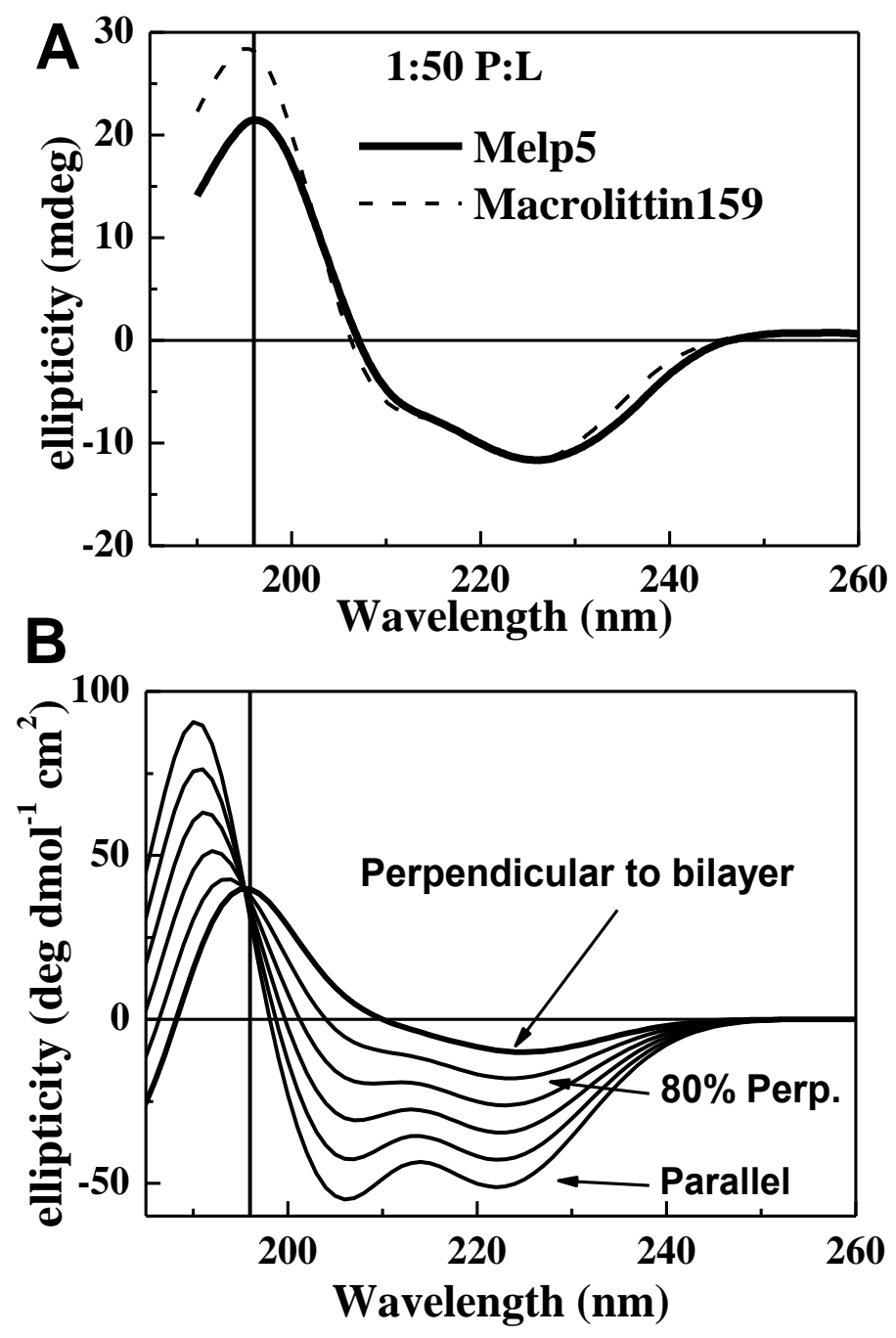

Figure 23 Oriented Circular Dichroism. A. OCD spectra are shown for macrolittin159 and for MeIP5 in comparison in stacked, oriented POPC multibilayers on a quartz substrate hydrated with water through the vapor phase. Spectra are the average of eight individual spectra collected at rotations around the beam axis. B. Basis theoretical OCD spectra, (top and bottom curve) and linear combinations for parallel and perpendicular helices calculated as described previously (REF). The four intermediate curves are for increments of perpendicular helix increasing by $20 \%$ per step.

Oriented circular dichroism spectroscopy. 
To determine the membrane orientation of the helices of membrane bound macrolittins family we used macrolittin159 as a representative of the family, and subjected it to oriented circular dichroism (OCD). Samples for OCD were hydrated stacked multilayers containing 20 mol\% peptide. The OCD spectra are shown in Figure 23 , and can be compared to the theoretical OCD spectra shown as a function of fraction of peptide inserted in a membrane spanning orientation. Comparison of the positions and ratios of the experimental macrolittin159 OCD spectral peaks to the theoretical peaks suggested that macrolittin159 is a membrane-spanning helix at $\mathrm{pH} 7$. We estimate an inserted fraction of at least $80 \%$. Previously, we showed that MelP5 is also a membranespanning helix under these conditions ${ }^{61}$. 

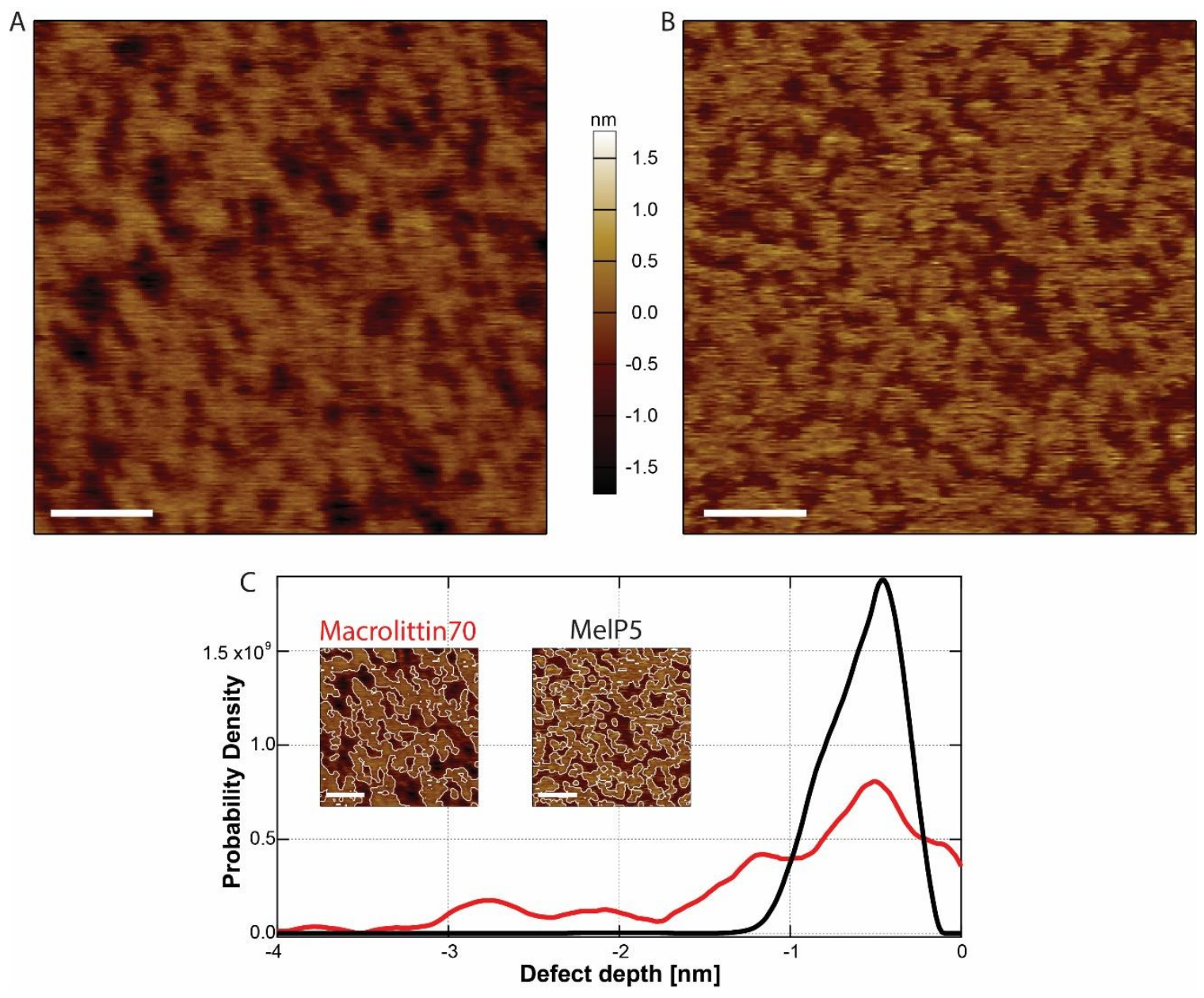

Figure 24 Membrane topography via atomic force microscopy. A. Representative AFM image of a POPC bilayer incubated with macrolittin70. B. For comparison, we show MelP5 under nominally identical conditions. C. Histograms of bilayer defect depths for both peptides (macrolittin70 in red, number of defects, $\mathbf{N}=378$; MelP5 in black, $N=1136$ ). Inset: Images with white contours outlining defect areas. For all images, scale bars (white) are $\mathbf{5 0 ~} \mathrm{nm}$.

Atomic Force Microscopy

We sought to directly observe the consequences of macrolittin-bilayer interactions via AFM imaging in physiologically relevant conditions: in fluid phase bilayers in aqueous solution at room temperature. We used macrolittin70 and compared the results to 
the membrane remodeling induced by MelP5. Prior to deposition onto freshly cleaved mica, vesicles of POPC were incubated with macrolittin70 at a starting P:L of 1:26. To remove loosely bound material, the samples were washed thoroughly with buffer before imaging. Nominally identical experiments using MelP5 were performed at the same P:L, and the effects of the two peptides on the bilayers was observed (Figure 24A \& B). The two peptides remodeled the bilayer in different ways. The macrolittin70-treated bilayer exhibited large and deep voids as well as numerous shallow defects. Some of these voids were deep and localized, suggestive of pores across the bilayer. MelP5-treated bilayers exhibited a single shallow defect mode. In order to delineate the effects of the two peptides further, a flood mask was used to isolate only the peptide affected areas of the bilayer (affected areas were defined as bilayer defects measuring at least $0.3 \mathrm{~nm}$ deep, Figure 24C: inset). Histograms of defect depths were calculated for the affected areas (Figure 24C). For macrolittin70, the histogram exhibited multiple sub-populations across a broad range of defect depths, whereas the MelP5 affected the bilayer in one prominent way - producing voids with depths roughly $0.6 \mathrm{~nm}$ below the top surface. We note that the upper surface in the AFM images may represent the unmodified upper leaflet of the bilayer; however, it is difficult to rule out the alternative possibility that the top surface could represent a peptide-distorted lipid layer. Regardless of the interpretation of the top surface, it is clear that macrolittin70 and MelP5 remodeled the lipid bilayer in different ways. Under essentially the same conditions, macrolittin70 had a more multifaceted and destructive effect on the lipid bilayer structure as compared to MelP5, consistent with the more potent membrane permeabilization activity described above. 


\section{Discussion}

Here we describe the macrolittins, a family of peptides that release macromolecules from lipid bilayers made from zwitterionic PC lipids at very low peptide concentration and at neutral $\mathrm{pH}$. Macrolittins readily enable the passage of a 40,000 $\mathrm{Da}$ dextran, a macromolecule with $4.5 \mathrm{~nm}$ hydrodynamic radius through lipid bilayers. Macromolecule release from liposomes is measurable at P:L as low as 1:5000 (20 peptides per vesicle) and reaches $50 \%$ at P:L as low as 1:1000, or 100 peptides per vesicle. Except for the pHD peptides at low pH (Figure 20B), such potent macromolecular poration by peptides in PC bilayers is unprecedented in the literature.

In the MelP5-based library from which both the macrolittins and the pHD peptides were selected, six positions had the possibility of containing an acidic aspartate or glutamate residue, or the native residue or, in some cases, another nonpolar residue (Figure 19). The selected macrolittins share a common, variable motif, always with three acidic amino acids per peptide, compared to five or six in all $\mathrm{pHD}$ peptides. In Figure 19D, we show the helical wheels of MelP5, the library, representative macrolittins, and representative pHD peptides. We note that these helical wheels may not be precisely correct because the Gly-Leu-Pro segment at residues 12-14 may be poorly structured and may lead to $\mathrm{N}$ - and $\mathrm{C}$-terminal helices that are somewhat independent of each other. Nonetheless, the helical wheels show that the library template sequence of MelP5 is highly amphipathic with a narrow polar face width. The library contains protonatable residues spread across a face that is much wider than the polar face of MelP5 and could potentially increase the polar face angle to almost $180^{\circ}$. While the polar face of the pHD peptides encompasses the largest widths that are encoded in the library, some of the 
acidic residues may be protonated at $\mathrm{pH} 5$ where the peptides are active. The macrolittins show a narrower polar face with acidic residues mostly in positions 4,8 and 11 or 15 , which comprise the center or the polar face. Residues 12 or 18 , which are the outsidemost two residues on the acidic face, are rarely found to be acidic in the macrolittins $(1 / 10)$, while they are acidic most of the time (17/20) in the pHD peptides.

The fact that two acidic residues at positions 4 and 8 are nearly $100 \%$ conserved in the five macrolittins (and also in the ten pHD peptides) may indicate that they are critical for the function that we observe for both families of peptides. The core of the polar face may be important for the structure that enables macromolecular poration. If so, the outermost residues of the wide polar face of the pHD peptides may be important for $\mathrm{pH}$ sensitivity. The conserved anionic residues at residues 4 and 8 are found on the same face of the N-terminal half of the helix. They are at about a $90^{\circ}$ angle on the helical wheel from either of the two positive residues, at residues 7 and 21 . The fact that we found lysine over histidine in these positions in 9/10 chances, indicates that having a positive charge at $\mathrm{pH} 7$ is important for macrolittin function. We speculate that parallel macrolittin helices have lateral electrostatic interactions between the conserved acidic groups and the basic amino acid at position 7 . Such a stabilized structure would create an amphipathic surface that could participate in a large pore. Antiparallel helices that are electrostatically stabilized by interactions between the $\mathrm{N}$-terminal acidic residues and the basic residue at position 21 are also possible, however the surface formed would be less amphipathic and thus seems less likely.

The ability to fold into an amphipathic $\alpha$-helix in membranes is a common structure-function relationship that exists for all of the generations of peptides discussed 
here, and for many other membrane permeabilizing peptides ${ }^{70}$. The original natural parent peptide, melittin, forms an amphipathic helix in membranes that lies mostly parallel to the membrane surface and creates transient pores at moderate concentrations ${ }^{17}$, 58-59, 71 . The synthetically evolved daughter sequence MelP5 has a more ideally amphipathic structure than melittin. It exists in a stable transmembrane configuration in PC bilayers and forms equilibrium pores ${ }^{61}$ that release macromolecules ${ }^{39}$. This behavior is consistent with the observation reported here that the macrolittins also have a transmembrane orientation. Transmembrane orientation is likely a critical structural requirement for macromolecular poration ${ }^{61}$.

We do not yet know the pore structure, but we can narrow the possibilities by considering minimal possible geometries. An extruded unilamellar vesicle is $50 \mathrm{~nm}$ in radius, has about 100,000 lipids and has a surface area of $\sim 3 \times 10^{4} \mathrm{~nm}^{\wedge} 2$. A dextran of 40 $\mathrm{kD}$ has a hydrodynamic radius of $\sim 4.5 \mathrm{~nm}$ meaning that a "pore" of at least $80 \mathrm{~nm}^{\wedge} 2(5$ $\mathrm{nm}$ radius, $1 / 300$ of the vesicle surface area) would be minimally required to pass it. Pores of this size are consistent with the AFM images presented in Figure 24. If we consider a classical barrel-stave pore, in which the peptides are laterally close-packed, the pore would have a circumference of at least $30 \mathrm{~nm}$, which would require $\geq 30$ peptides to line it. Thus, a lipid vesicle can have only a few pores at P:L 1:1000 (100 peptides per vesicle), where activity is high.

With such large pathways across the bilayer, it may be more fruitful to consider a structural model in which these amphipathic peptides drive bilayer thinning and stabilize exposed bilayer edges by reducing line tension at low concentration as other amphipathic peptides, proteins, polymers and detergents do at higher concentration ${ }^{72-75}$. The AFM 
results are consistent with the macrolittins having a dramatic effect on overall bilayer thickness and ${ }^{76}$ structure. In this scenario, individual peptides are not in direct contact with each other, but instead cooperatively alter lipid thickness and curvature to stabilize an exposed bilayer edge in a so-called toroidal pore geometry ${ }^{77-79}$. In this architecture, electrostatic stabilization remains possible because such interactions have a long range. Fewer peptides could be needed to stabilize such a toroidal "pore". The critical interactions, in this case, would be curvature-dependent interactions between the bilayers that may work synergistically with electrostatic interactions between peptides.

Acknowledgements: Funded by NIH R01 GM111824 and NSF DMR 1710053

(WCW), NSF DMR 1709892 (KH) and NSF DMR 1709792 (GMK). 


\title{
Project Three: pHD108
}

\author{
Mechanism of action of peptides that cause pH-triggered macromolecular poration of lipid \\ bilayers \\ Kim, Sarah; Pittman, Anna; Zapata-Mercado, Elmer; King, Gavin; Wimley, William; Hristova, \\ Kalina \\ Journal of the American Chemical Society Article ASAP
}

\section{Introduction}

Membrane active, $\mathrm{pH}$-sensitive peptides can be utilized for delivering cargo to cells through endosomes ${ }^{76,80-81}$, or for new therapies that target cancer cells ${ }^{82-84}$, among other applications. Although the mechanism of action of several such $\mathrm{pH}$-sensitive peptides have been studied, the peptides previously investigated either insert as monomers without pore formation (i.e. pHLIP), or form only small pores in membranes (e.g. GALA) $)^{85-86}$. Large pores are needed for most applications. The mechanism of action of $\mathrm{pH}$-sensitive peptides that form large, macromolecule-sized pores will likely involve additional factors such as cooperativity and peptide-peptide interactions. By rationally designing an iterative peptide library, and using a high-throughput functional screen, we have previously discovered a family of peptides that cause macromolecular poration in synthetic membranes at low concentration, in a way that is triggered by acidic $\mathrm{pH}^{23}$. These peptides, which we named the $\mathrm{pH}$-dependent Delivery (pHD) peptides, are unprecedented in their ability to cause highly efficient, $\mathrm{pH}$-triggered leakage of large molecules through synthetic membranes. At $\mathrm{pH} 7$ or above, the members of this synthetically evolved family of sequences are essentially inactive; they induce little release of small or large molecules from lipid bilayer vesicles. Yet, at $\mathrm{pH} \leq 6$, they bind 
to membranes, fold into $\alpha$-helices, and enable efficient release of macromolecules at very low peptide concentration. To understand the mechanism of action of the pHD peptides, here we asked how peptide density on membrane surfaces and cooperativity between peptides promote macromolecule-sized pore-formation. Furthermore, we asked how pH regulates these processes. To answer these questions, we examined the structure of the pores formed, and we systematically explored structure-function relationships through measurements of the effect of $\mathrm{pH}$ and peptide concentration on membrane binding, peptide structure, and the formation of large pores by the pHD peptides in membranes.

\section{Materials and Methods}

Peptides of $>95 \%$ purity were purchased from BioSynthesis, Inc. 1-palmitoyl-2oleoyl-glycero-3- phosphocholine (POPC) and 1-palmitoyl-2-(9,10-dibromo)stearoyl-snglycero-3-phosphocholine (diBrPSPC) was purchased from Avanti Polar Lipids. 8Aminonaphthalene-1,2,3-trisulfonic acid (ANTS) and p-xylylenebis(pyridinium bromide) (DPX) were purchased from Thermo Fisher Scientific. Chloroform, ammonium thiocyanate, and other salts and buffer materials were purchased from Fisher Scientific or Sigma-Aldrich. TBD was synthesized as described elsewhere ${ }^{23,39}$.

Buffers. Thirteen buffers were prepared with $\mathrm{pH}$ from 4 to 7 at $0.25 \mathrm{pH}$ increments. Buffers with a pH between 4 and 5.5 were prepared with $10 \mathrm{mM}$ sodium acetate, and buffers with a $\mathrm{pH}$ between 5.75 and 7 were prepared with $10 \mathrm{mM}$ sodium phosphate. Buffers for binding and leakage assays were prepared with $100 \mathrm{mM} \mathrm{KCl}$. Buffers for circular dichroism were prepared without $\mathrm{KCl}$, and their $\mathrm{pH}$ was adjusted with phosphatidic acid instead of hydrochloric acid to minimize the CD signal due to the 
absorption of the chloride ion below $200 \mathrm{~nm}$. All buffers were vacuum filtered through a $0.22 \mu \mathrm{m}$ pore size membrane to remove dust and bacteria before use.

Peptides. Solutions of $\sim 1 \mathrm{mM}$ peptides were prepared with Millipore water. Concentrations were determined using the absorbance of the single tryptophan on each peptide. The average of three absorbance measurements at $280 \mathrm{~nm}$ on a NanoDrop 2000c was used to determine the concentration. Peptides were stored frozen until use and were subjected to no more than five freeze-thaws to minimize peptide degradation.

Vesicle Preparation. Three types of $100 \mathrm{~nm}$ large unilamellar vesicles (LUV) were prepared with synthetic POPC lipids. For all vesicle types, POPC in chloroform was under vacuum overnight, resuspended in buffer and extruded ten times through $100 \mathrm{~nm}$ poly carbonate membranes. Plain vesicles were used for binding and circular dichroism. For TBD vesicles, dry lipid films were resuspended in buffer containing $1 \mathrm{mg}$ TBD per $50 \mu$ mol lipid. After extrusion, vesicles were incubated on high capacity streptavidin agarose to remove unencapsulated TBD. For ANTS/DPX vesicles, dried lipid films were resuspended in $12.5 \mathrm{mM}$ ANTS and $45 \mathrm{mM}$ DPX. After extrusion, unencapsulated ANTS and DPX were separated from the vesicles by size exclusion chromatography with Sephadex G-100 resin. Lipid concentration was measured using a modified Stewart Assay ${ }^{66}$.

Dextran leakage assays. Leakage of $40 \mathrm{kDa}$ was measured with FRET. Dextran vesicles with entrapped TBD were diluted to $1 \mathrm{mM}$, and streptavidin-AF488 (the donor fluorophore) was added to a final concentration of $20 \mathrm{nM}$. In a 96-well plate, peptide and vesicles were mixed, then incubated while shaking at room temperature for 1 hour before 
measuring FRET by donor fluorescence quenching on a BioTek H4 Synergy Hybrid Microplate Reader with ex/em $=495 / 519 \mathrm{~nm}$. As a positive control for $100 \%$ leakage, 4 $\mu \mathrm{l}$ of $10 \%$ Triton X100 was added to three wells, and as a negative control, no peptide was added to three wells. Leakage measurements are the average of at least 3 unique vesicle preparations. Fractional leakage was calculated as:

$$
f_{\text {dextran leakage }}=\left(F_{\text {no peptide }}-F_{\text {sample }}\right) /\left(F_{\text {no peptide }}-F_{\text {triton }}\right)
$$

The leakage as a function of $\mathrm{pH}$ was fit with a sigmoidal curve to determine the midpoint, or $\mathrm{pH} 50$.

ANTS/DPX Leakage assays. Small molecule leakage was measured by quenching of ANTS by DPX. ANTS/DPX leakage vesicles were diluted to $1 \mathrm{mM}$. On a 96-well plate, peptide and vesicles were mixed at P:L ranging from 1:50 to 1:5000, then incubated with shaking at room temperature for 1 hour before measuring ANTS fluorescence using a microplate reader with ex/em $=360 / 519 \mathrm{~nm}$. As a positive control for $100 \%$ leakage, $4 \mu \mathrm{l}$ of $10 \%$ Triton X100 was added to three wells, and as a negative control, no peptide or Triton was added to three wells. Leakage measurements are the average of at least 3 unique vesicle preparations. Fractional leakage was calculated as:

$$
f_{\text {ANTS } / \text { DPX leakage }}=\left(F_{\text {sample }}-F_{\text {no peptide }}\right) /\left(F_{\text {triton }}-F_{\text {no peptide }}\right)
$$

The leakage as a function of $\mathrm{pH}$ was fit with a sigmoidal to determine the midpoint of the curve, or the $\mathrm{pH} 50$.

Tryptophan Binding. POPC vesicles were prepared at $\sim 30 \mathrm{mM}$ at $\mathrm{pH} 4.75$ and $\mathrm{pH}$ 6.25 as described above. Vesicles were diluted to $1 \mathrm{mM}$ with buffers spanning $\mathrm{pH} 4$ to 7 , 
then incubated overnight at $4^{\circ} \mathrm{C}$ to allow the internal and external $\mathrm{pH}$ to equilibrate while minimizing vesicle degradation due to hydrolysis. The next morning, the $\mathrm{pH}$ of the solution was verified with MColorpHast $\mathrm{pH}$ test strips. Peptide was added with P:L ranging from 1:50 to 1:5000 in $0.5 \mathrm{~mL}$ vials. After 1 hour of incubation at room temperature, tryptophan fluorescence spectra were measured on a Horiba Fluorolog 3-22 in a $45 \mu \mathrm{l}$ cuvette. Samples to correct for lipid scattering, including vesicles, vesicles with tryptophan (at P:L ranging from 1:50 to 1:5000), and tryptophan (at P:L ranging from 1:50 to 1:5000), were also measured at each $\mathrm{pH}$, but without a 1 hour incubation. Scattering correction was made as described ${ }^{69}$. The midpoint of the curve, or the pH50, was obtained by fitting with a sigmoidal curve.

Circular Dichroism. POPC vesicles without $\mathrm{KCl}$ were prepared as described above for the tryptophan binding. Scans were collected in a $1 \mathrm{~mm}$ pathlength cuvette on a Jasco J-715 spectropolarimeter with a scan rate of $100 \mathrm{~nm} / \mathrm{min}, 3$ accumulations, at room temperature. After measurement, the scans were corrected for background using a vesicle sample with no peptides. Relative \% helicity was calculated by scaling the average ellipticity at $222 \mathrm{~nm}$. The midpoint of the curve, or the pH50, was obtained by fitting with a sigmoidal curve.

Tryptophan Quenching. To determine if the pHD peptides can exchange from vesicles to which they are bound into a second population of vesicles added after binding, we measured the change in tryptophan fluorescence upon the addition of a second population of vesicles composed of brominated lipids. Since bromines quench tryptophan fluorescence ${ }^{69}$,the exchange of peptides from POPC to brominated vesicles will result in quenching. We prepared vesicles composed of POPC or 16:0-18:0 (9,10 diBr) $\mathrm{PC}$ at $\mathrm{pH}$ 
4.5 , and diluted them to $1 \mathrm{mM}$. To determine the extent of tryptophan quenching by the brominated vesicles, $100 \mu \mathrm{l}$ of $1 \mathrm{mM}$ diBrPSPC was mixed with peptide added to a final concentration of $20 \mu \mathrm{M}$ (P:L = 1:50). After 1 hour, the tryptophan fluorescence spectra were measured as described in the binding assay. To measure the kinetics of quenching, the tryptophan fluorescence of pHD15 was measured at 280/334nm. After adding $100 \mu 1$ of $1 \mathrm{mM}$ POPC to a cuvette, fluorescence was measured for up to 5 minutes, and then peptide was added up to a final concentration of $20 \mu \mathrm{M}$. After waiting 30 minutes to ensure maximal binding to the vesicles, diBrPSPC or POPC (as a negative control) was added to final concentrations of 0.2 or $0.33 \mathrm{mM}$, and fluorescence was measured for 30 more minutes.

Sample Preparation for Atomic Force Microscopy (AFM) Imaging. We adopted established methods to form supported lipid bilayers on mica surfaces ${ }^{50}$. Briefly, POPC was suspended in $300 \mathrm{mM} \mathrm{NaCl}, 40 \mathrm{mM} \mathrm{CaCl}$, and $20 \mathrm{mM}$ HEPES, $\mathrm{pH}$ 4, at a concentration of $600 \mu \mathrm{M}$. Liposomes were prepared by extrusion of POPC through a polycarbonate membrane with a $100 \mathrm{~nm}$ pore diameter. The peptide was diluted to a concentration of $10 \mu \mathrm{M}$ in imaging buffer: $200 \mathrm{mM} \mathrm{NaCl}, 50 \mathrm{mM}$ HEPES at $\mathrm{pH} 4$. POPC liposomes were incubated for $10 \mathrm{~min}$ in solution with the peptide to yield a final peptide concentration of $3 \mu \mathrm{M}$. The liposomes containing peptides were then deposited onto freshly cleaved mica. Supported bilayers were formed by vesicle fusion (30 min incubation, $\left.\sim 30{ }^{\circ} \mathrm{C}\right)$. Samples were rinsed four times using $75 \mu \mathrm{L}$ of imaging buffer. Due to rinsing and other factors, P:L assignment during AFM imaging is challenging; we estimate a P:L of roughly 1:100 for all data shown here. For experiments at $\mathrm{pH}>4$, a volume of $200 \mathrm{mM} \mathrm{NaCl}, 50 \mathrm{mM}$ Hepes, $\mathrm{pH} 7$ was added to the supported lipid bilayer, 
resulting in a final $\mathrm{pH}$ of approximately 6 . All AFM data were collected at $\sim 32{ }^{\circ} \mathrm{C}$, which is significantly above the gel-to-fluid transition temperature of POPC.

AFM Imaging and Analysis. Images were acquired in tapping mode in aqueous buffer solution using a commercial apparatus (Asylum Research Inc., Cypher) and Biolever mini tips with spring constant $\mathrm{k} \approx 0.9 \mathrm{~N} / \mathrm{m}$ (Olympus, BL-AC40TS). Care was taken to control the magnitude of the tip-sample force to $\leq 100 \mathrm{pN}$ (estimated by comparing the free amplitude to the set point amplitude). As is typical, images were flattened (second order) to minimize background. For statistical analysis, the data were offset so as to align the population having the greatest height, corresponding to the top surface in each image, to zero. For depth measurements we assumed that the top surface represented the unmodified surface of the upper leaflet of the supported bilayer; however, is difficult to rule out the formal possibility that this top surface is itself modified by the presence of pHD108 peptides. Individual pore-like features were delineated using custom software which uses the Hessian blob strength to identify features from the background, and reported the footprint area of each feature ${ }^{47}$. The depth of the features was determined using software (Igor Pro, WaveMetrics). Smoothed histograms (kernel density estimates, generated in Igor Pro) were normalized to unity when the abscissa (and ordinate, for the two dimensional case) was expressed in mks units. Data were fit with Gaussians (MagicPlot), with defined as the center of the peak.

\section{Results}

\section{Peptides Selected for Study}


Here, we selected three pHD peptides for detailed characterization. The peptides pHD24 (GIGDVLHELAADLPELQEWIHAAQQL) and pHD108 (GIGEVLHELAEGLPELQEWIHAAQQL) were selected because they are canonical sequences, with 5 acidic residues like most of the active sequences identified. These two peptides had similarly potent activity, and similar pH50 values in our preliminary studies $^{23}$. pHD24 has 3 aspartate residues and two glutamates, the more common acidic residue found across the family, while pHD108 has 5 glutamates. The peptide pHD15 (GIGEVLHELADDLPDLQEWIHAAQQL) is an outlier which has 6 acidic residues. Its potency is somewhat lower, and it becomes active at a somewhat lower $\mathrm{pH}$ than the others. We include it here to enable characterization across a range of potencies and $\mathrm{pH}$ dependences.
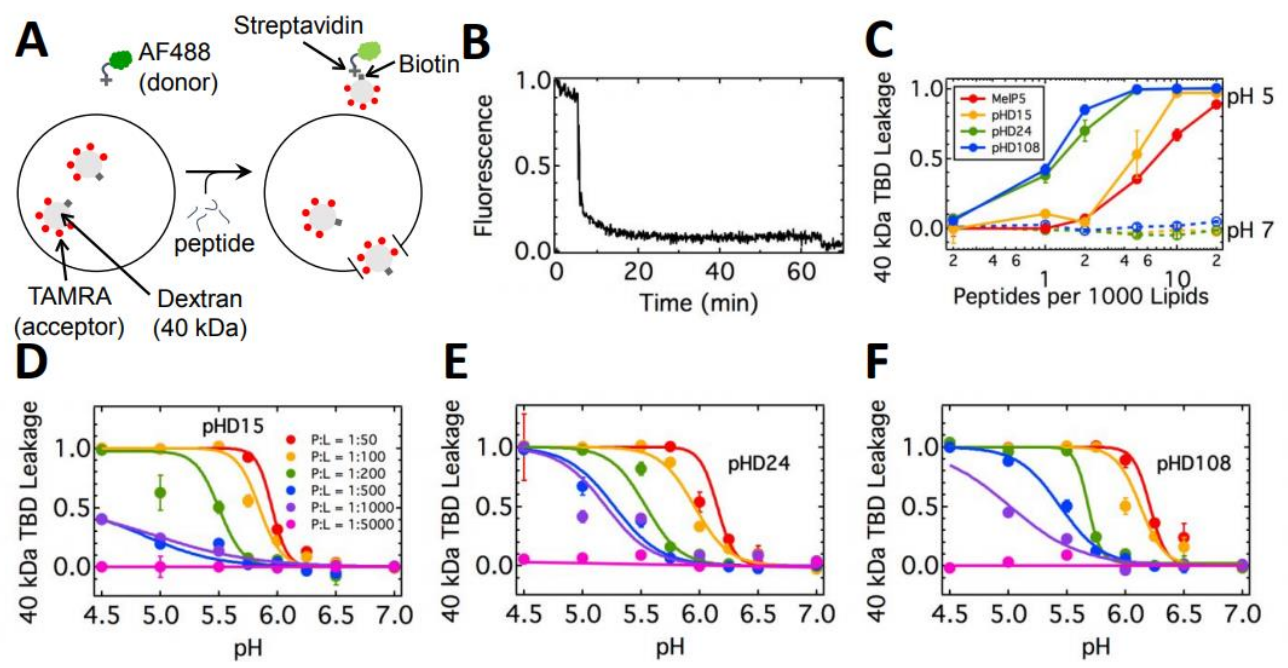

Figure 25 Macromolecular Leakage Assay. (A) Schematic of the assay. Peptides were incubated with streptavidin-AlexaFluor488 and POPC vesicles containing TAMRA-biotin-dextran (TBD) for one hour. Upon peptide-mediated macromolecular poration, the 40 kDa dextran can leak 
out of the vesicles and form a complex with streptavidin, leading to FRET. (B) A time course of streptavidin-AlexaFluor488 (donor) intensity upon TBD vesicle permeabilization. At about $t=5$ min, pHD108 (P:L = 1:100) was added to a solution of $1 \mathrm{mM}$ POPC at pH 6. After 1 hour, Triton X100 was added for the $100 \%$ leakage fluorescence value. (C) The extent of dextran leakage as a function of concentration at pH 5 and pH 7. At pH 5, the pHD peptides are more potent than

MelP5. At pH 7, the pHD peptides are not active, even at the highest concentrations measured. (D, E and F) Dextran leakage as a function of pH and P:L for pHD15 (D), pHD24 (E), and pHD108 (F).

\section{Macromolecular poration by the $\mathbf{p H D}$ peptides}

Using the same fluorescence-based assay we developed for the screen in which we discovered the pHD peptides ${ }^{23}$, we characterized the ability of pHD15, pHD24, and pHD108 to form large pores in vesicles as a function of both $\mathrm{pH}$ and peptide concentration. Results from these assays are shown in Figure 25. Figure 25A shows a schematic of the assay. The entrapped probe used to detect macromolecule leakage was a 40,000 Da dextran labelled with the dye TAMRA (fluorescence acceptor) and with biotin (called "TBD" for TAMRA-biotin-dextran). The external solution contained streptavidin labelled with Alexafluor488 (fluorescence donor). In this assay, leakage of encapsulated TBD leads to streptavidinTBD complex formation and quenching of Alexafluor488 due to $\mathrm{FRET}^{39,87}$. The hydrodynamic radius of dextran is about $4.5 \mathrm{~nm}^{88}$, and therefore a pore with a radius of at least $45 \AA$ is required to form in the bilayer, for dextran leakage to occur. In Figure 25B, we show the kinetics of TBD leakage. Upon addition of peptide to solution of vesicles at $\mathrm{pH}$, the donor fluorescence drops steeply and reaches a plateau. Leakage is rapid, with greater than $90 \%$ leakage occurring over 10 minutes. In Figure $25 \mathrm{C}$ we show the fractional leakage of TBD, measured at 60 minutes, as a function of peptide-to-lipid ratio (P:L) at pH 5. pHD24 and pHD108 are very potent at pH 5, with 
substantial activity observed at concentrations as low as 1 peptide per 1000 lipids, and $\geq 95 \%$ activity at $\mathrm{P}: \mathrm{L}=1: 200$. pHD15 is less active, with $\geq 95 \%$ activity occurring at about $\mathrm{P}: \mathrm{L}=1: 100$. MelP5, the parent peptide used for library design, is less active than pHD15 with $\geq 95 \%$ activity at $\mathrm{P}: \mathrm{L}>1: 50$. No leakage is observed at $\mathrm{pH} 7$ for any of the pHD peptides, even at the highest concentrations measured. In Figure 25D-F, we show the leakage as a function of $\mathrm{pH}$ for $\mathrm{pHD} 15, \mathrm{pHD} 24$, and $\mathrm{pHD} 108$. At the lowest concentrations, little or no leakage is observed at any $\mathrm{pH}$. At the highest concentrations, leakage sharply transitions to $100 \%$ with decreasing $\mathrm{pH}$. Under these conditions, the transition as a function of $\mathrm{pH}$ is steep, occurring over $1 \mathrm{pH}$ unit. Such steepness likely indicates that the acidic residues protonate and deprotonate cooperatively ${ }^{89}$. Interestingly, we observe a large increase in pH50 as peptide concentration is increased. Peptides pHD24 and pHD108 experience a shift of about one $\mathrm{pH}$ unit when the peptide concentration is increased from P:L of 1:500 to 1:50. For pHD15, the shift is somewhat smaller at P:L where it can be compared. 
A

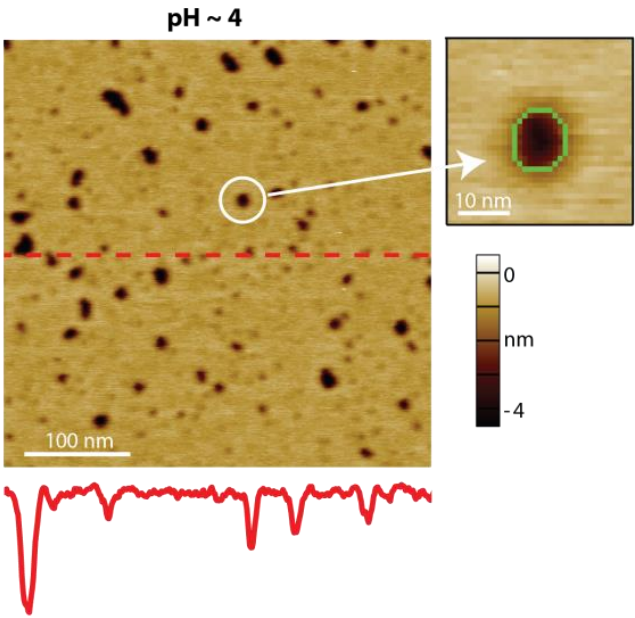

B

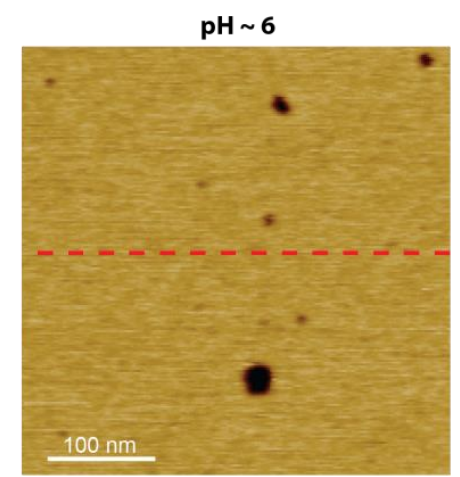

ำ
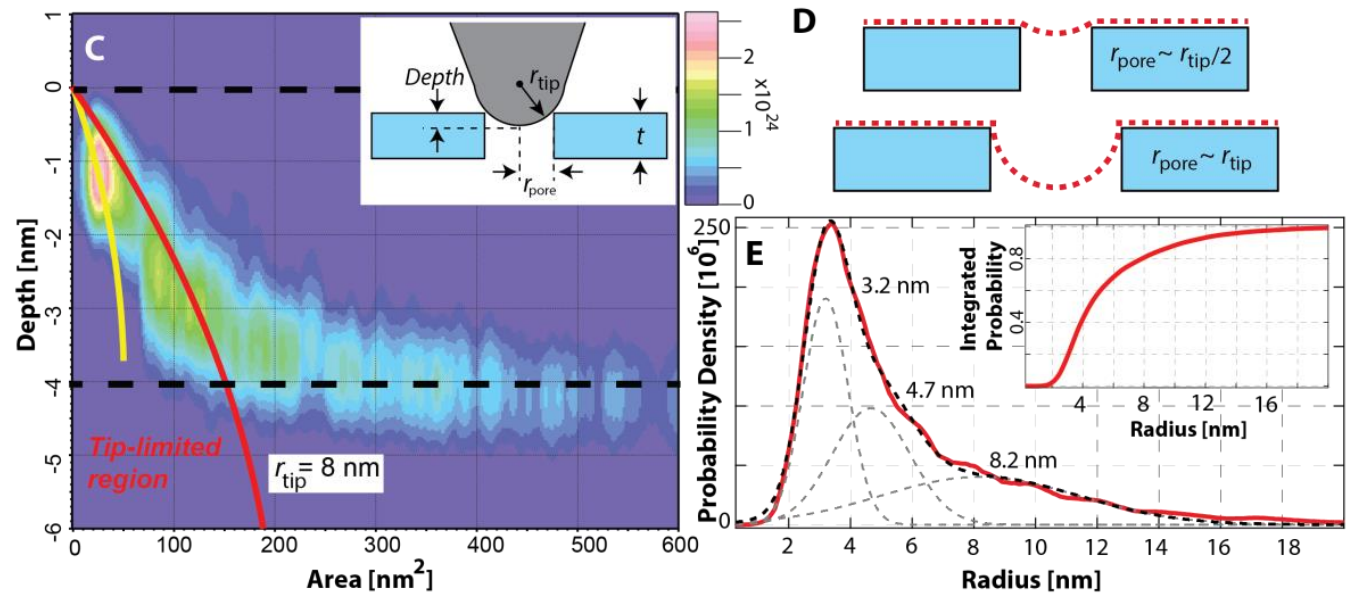

Figure 26 Membrane topography via atomic force microscopy. (A) AFM image at pH 4 showing a variety of punctate pore-like features in a POPC bilayer incubated with pHD108. A line scan is shown below the image, position indicated by red dashed line. A detailed view of the feature circled in white shows the result of the Hessian blob algorithm (green perimeter). The area defined by this perimeter was used to compute effective radius. (B) Image at pH 6 shows significantly fewer features. (C) Bivariate heat map of feature depth versus area at $\mathrm{pH} 4$. The red and yellow overlaid curves show tip-limited regions for rtip = 8 and $4 \mathrm{~nm}$, respectively, where a small radius pore would read artificially shallow, as shown in the inset. Dashed black lines represent the top and bottom of the bilayer surface. Increasing probability density is shown in false color from purple to red. (D) Cartoons demonstrate how two equally deep pores spanning the entire thickness of the membrane, $t$, could result in two different depth measurements (here rtip $=2 \mathrm{t}=8 \mathrm{~nm}$ ). (E) Distribution of effective radii of features constructed 
from analyzing over 20,000 features (data are red, fits are dashed). Inset: Integrated radii distribution.

To further characterize the bilayer poration induced by the pHD peptides, we used atomic force microscopy (AFM). AFM can provide a direct visualization of peptideinduced topographical structure in supported lipid bilayers. Following recent studies on the parent peptide MelP5 and the macrolittins ${ }^{21-22}$, we applied tapping mode AFM to image pHD108-induced membrane remodeling. Peptides and POPC vesicles were incubated prior to deposition onto freshly cleaved mica and then imaged in solution at an approximate P:L of 1:100. Data acquired at $\mathrm{pH} 4$ exhibited numerous punctate pore-like features (Figure 26A). Interestingly, when the $\mathrm{pH}$ of the imaging buffer was raised, the surface topography of the sample changed substantially. In particular, fewer punctate pore-like features were observed at $\mathrm{pH} 6$ (Figure 26B) compared to $\mathrm{pH} 4$. The average number of features was determined in 12 non-overlapping $500 \times 500 \mathrm{~nm} 2$ areas. The results indicate a six-fold reduction in average number of features from 248 (at $\mathrm{pH} 4$ ) to 40 (at $\mathrm{pH}$ 6). If we restrain this analysis to features exhibiting effective radii smaller than $8 \mathrm{~nm}$, to exclude large voids or membrane defects, then there is an approximate order of magnitude reduction in average number of punctate pore-like features per area, from 215 ( $\mathrm{pH} 4)$ to 20 ( $\mathrm{pH} \mathrm{6).} \mathrm{A} \mathrm{few} \mathrm{large} \mathrm{voids} \mathrm{(or} \mathrm{defects)} \mathrm{in} \mathrm{the} \mathrm{bilayer} \mathrm{were} \mathrm{observed} \mathrm{at} \mathrm{both}$ pH 4 and pH 6 and were relatively stable. Regardless of the metric, the change in $\mathrm{pH}$ had a marked effect on peptide remodeling of the lipid bilayer. We note that it is unlikely that the act of exchanging buffer itself would have such an effect on the lipid bilayer, as the bilayer sample was washed repeatedly $(4 \mathrm{x}$ with $75 \mathrm{uL})$ with the $\mathrm{pH} 4$ buffer prior to 
imaging. We note that in the absence of peptide, the upper leaflet surface of a POPC bilayer is essentially featureless ${ }^{21}$.

To quantify pore-like structures in a statistical manner, features were detected algorithmically from a series of AFM images at $\mathrm{pH} 4$. Figure $26 \mathrm{C}$ shows a bivariate histogram of feature depths verses footprint area. Dashed black lines indicate the top and bottom of the bilayer, and overlaid red and yellow curves show tip-limited regions for two different assumed radii rtip $=8$ and $4 \mathrm{~nm}$, respectively. Features that lay within this region have shallower depth readings, most likely due to the feature being too narrow for the tip to penetrate to the bottom, as shown by the cartoon inset. Features with larger areas tended to have deeper depths, approaching the expected $4 \mathrm{~nm}$ thickness of the membrane. Figure 26D demonstrates how two pores, both of which span the membrane, could result in two different depth measurements: one accurate, and one artificially shallow. We also report the effective radius distribution of features (Figure 26E) as determined from Hessian blob analysis under the assumption of circularity (which matched many of the features). Three populations of radius were prominent: $3.2 \mathrm{~nm}, 4.7$ $\mathrm{nm}$, and $8.2 \mathrm{~nm}$. Integration of the radius distribution (Figure 26E, inset) indicated that approximately $50 \%$ of total features exhibited a radius $\geq 4.5 \mathrm{~nm}$, consistent with leakage of $40 \mathrm{kDa}$ dextran (Stokes' radius $\sim 4.5 \mathrm{~nm}$ ). This analysis, coupled with the observation of fewer features observed at higher $\mathrm{pH}$, corroborates the aforementioned macromolecular poration studies and further suggests that many of the features identified via AFM are bonafide pores spanning the thickness of the membrane. 

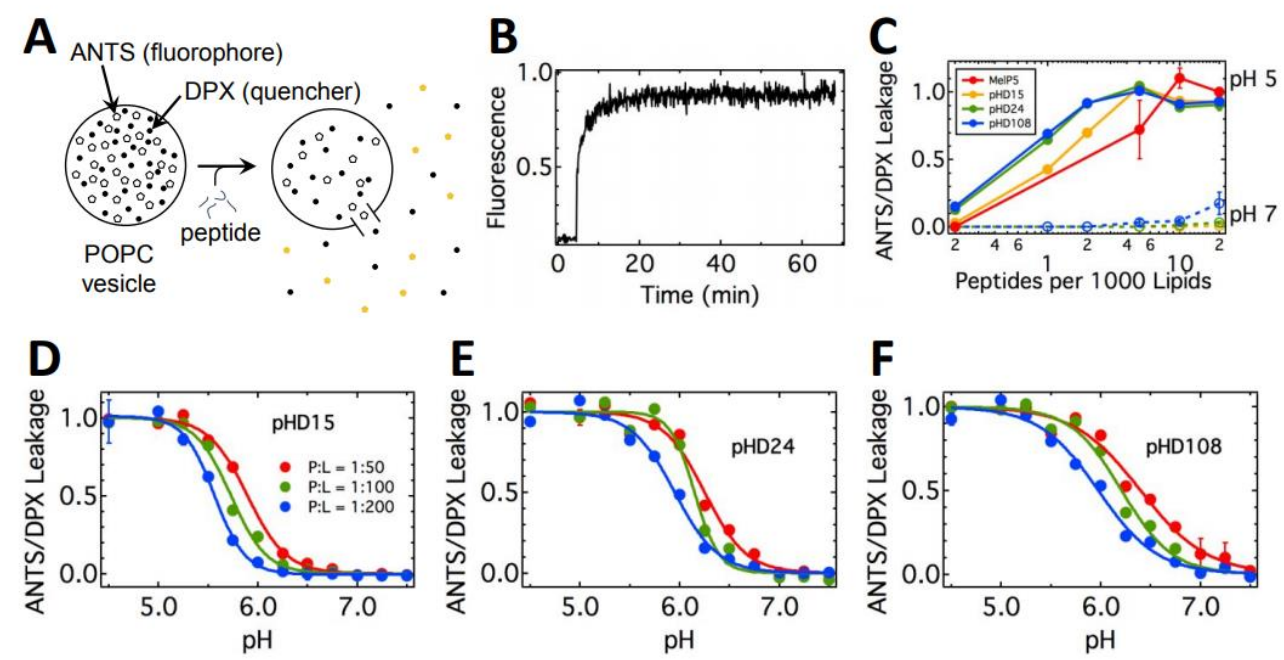

Figure 27 ANTS/DPX Leakage Assay. (A) Schematic of the assay. Peptides were incubated with POPC vesicles with encapsulated ANTS (fluorophore) and DPX (quencher) for 1 hour. Upon pore formation, dilution of ANTS and DPX results in an increase of ANTS fluorescence. (B) Time course of ANTS leakage by pHD108 at pH 5. ANTS fluorescence at was monitored on a fluorimeter $(e x / e m=350 / 519 \mathrm{~nm})$. At about $\mathrm{t}=3 \mathrm{~min}$, peptide was added to a cuvette with 1 mM POPC vesicles for a final P:L of 1:100. After 1 hour, Triton X100 was added to obtain the $100 \%$ leakage fluorescence value. (C) Leakage as a function of P:L. At pH 5, the pHD peptides are more potent than MelP5. At pH 7, the peptides do not cause much leakage, even at the highest concentrations measured. (D, E and F) Leakage as a function of pH for pHD15 (D), pHD24 (E) and pHD108 (F).

\section{Small molecule release in response to the $\mathrm{pHD}$ peptides}

To determine if the $\mathrm{pHD}$ peptides form small pores at low peptide concentrations, we measured pore formation with an ANTS/DPX leakage assay, which detects the leakage of $\sim 0.4 \mathrm{kDa} \mathrm{ANTS}^{90}$. Results from these assays are shown in Figure 27. Vesicles containing co-encapsulated fluorophore (ANTS) and quencher (DPX) were incubated with peptide for 1 hour. Figure 27A shows a schematic of the leakage assay. Crowding of 
ANTS and DPX within the vesicles quenches ANTS fluorescence. Pore-formation results in ANTS/DPX release and a concomitant increase in ANTS fluorescence. In Figure 27B, we show the kinetics of leakage. Upon addition of peptide, ANTS fluorescence increases sharply, then reaches a plateau. Release of ANTS and DPX is rapid, and very similar to TBD leakage, with greater than 90\% leakage occurring over 10 minutes. In Figure 27C we show fractional leakage of ANTS and DPX, measured at 60 minutes, as a function of peptide-to-lipid ratio. The peptides are very potent at $\mathrm{pH} 5$, with substantial activity observed at concentrations as low as $\mathrm{P}: \mathrm{L}=1: 1000$, and $\geq 95 \%$ activity at $\mathrm{P}: \mathrm{L}=1: 200$. Small molecule release is more potent than macromolecule release (Figure 25). In small molecule leakage, as with macromolecule leakage, pHD15 is less active than pHD24 and pHD108, and MelP5 is less active than pHD15, but the differences are smaller than for macromolecule release. Little or no ANTS leakage is observed at $\mathrm{pH} 7$ for the $\mathrm{pHD}$ peptides, even at the highest concentrations measured. In Figure 27D-F, we show the leakage as a function of $\mathrm{pH}$ for $\mathrm{pHD} 15, \mathrm{pHD} 24$, and $\mathrm{pHD} 108$. At the concentrations shown, leakage sharply transitions to $100 \%$ with decreasing $\mathrm{pH}$. The transition as a function of $\mathrm{pH}$ is steep. Again, $\mathrm{pH} 50$ increases as peptide concentration is increased, with a shift of about $0.3 \mathrm{pH}$ units when the peptide concentration is increased from $\mathrm{P}: \mathrm{L}=$ 1:200 to 1:50. For pHD24 and pHD108, the pH50 increases from 6.0 to 6.4 . For pHD15, the pH50 is lower; it increases from 5.6 to 5.9 as peptide concentration is increased from 1:200 to 1:50. 

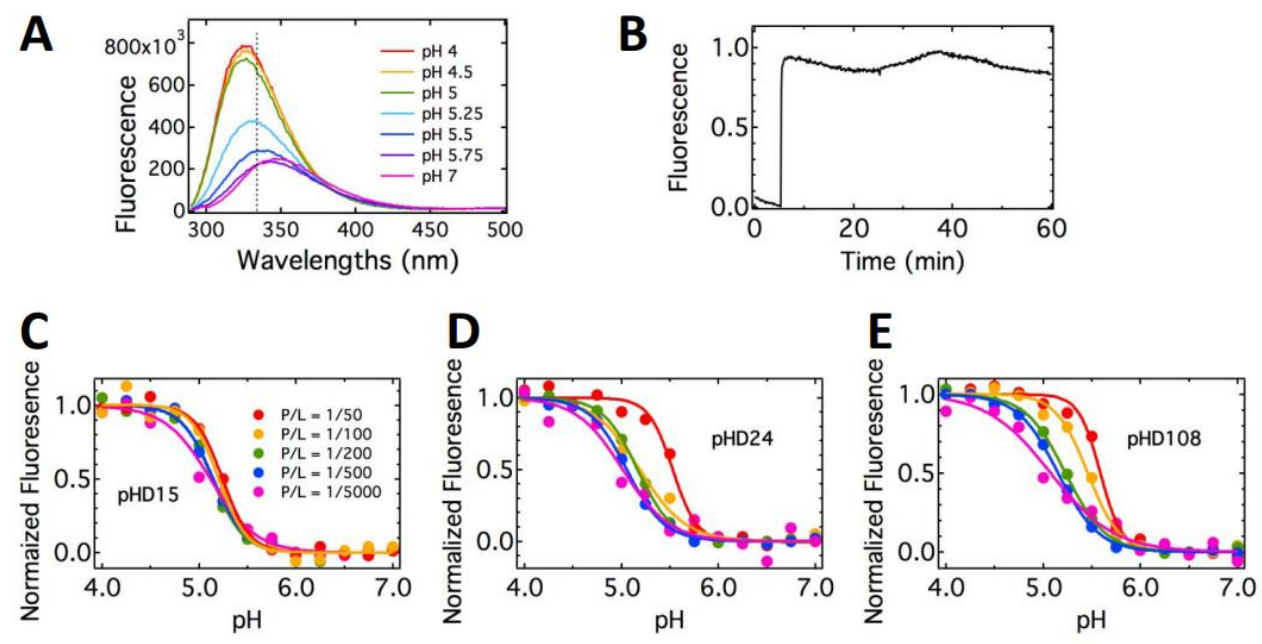

Figure 28 pHD peptide binding to lipid bilayers, as followed by tryptophan fluorescence. The pHD peptides each have one tryptophan, and its fluorescence is sensitive to the polarity of its environment. (A) Fluorescence spectra of pHD15 as a function of pH ranging from 4 to 7. Samples with $10 \mu \mathrm{M}$ pHD15 and $1 \mathrm{mM}$ POPC (P:L = 1:100) were incubated for 1 hour before spectra were measured. The dotted line is at $334 \mathrm{~nm}$. (B) A time course of pHD108 tryptophan fluorescence. Upon addition of vesicles at about $t=5 \mathrm{~min}$, binding is rapid. (C, D, E) Normalized fluorescence as a function of pH for pHD15 (C), pHD24 (D), and pHD108 (E).

\section{Binding of the $\mathrm{pHD}$ peptides to membranes}

We characterized the binding of the peptides as a function of $\mathrm{pH}$ and peptide concentration with tryptophan fluorescence titration ${ }^{69}$. As shown in Figure 28A, the fluorescence emission maximum shifts from $\sim 350 \mathrm{~nm}$ at neutral $\mathrm{pH}$ to $330 \mathrm{~nm}$ at acidic $\mathrm{pH}$, indicative of a transition from aqueous, exposed tryptophan to membrane-inserted $\operatorname{tryptophan}^{91}$. In Figure 28B, we show a kinetic trace of binding at $\mathrm{pH} 4.5$, under conditions at which the peptide is active. Upon addition of peptide to a $1 \mathrm{mM}$ vesicle solution at $\mathrm{t}=8$ minutes, binding occurs rapidly, and is essentially complete in 1 minute. In Figure 28C-E, we show the scaled fluorescence intensities at $335 \mathrm{~nm}$ as a function of 
$\mathrm{pH}$ for different peptide concentrations for $\mathrm{pHD} 15, \mathrm{pHD} 24$, and $\mathrm{pHD} 108$. In all cases, binding is dependent on $\mathrm{pH}$ with a sharp transition occurring over about $1 \mathrm{pH}$ unit, which is indicative of cooperativity. Peptides pHD108 and pHD24 show a small increase in $\mathrm{pH} 50$ and a sharper slope as peptide concentration is increased. The $\mathrm{pH} 50$ increases from 5.1 to 5.6 for pHD108, and from 5.0 to 5.5 for pHD24 as P:L increases from P:L of 1:5000 to $1: 50$.

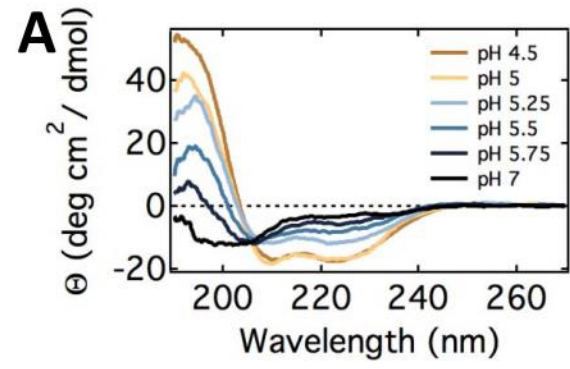

B
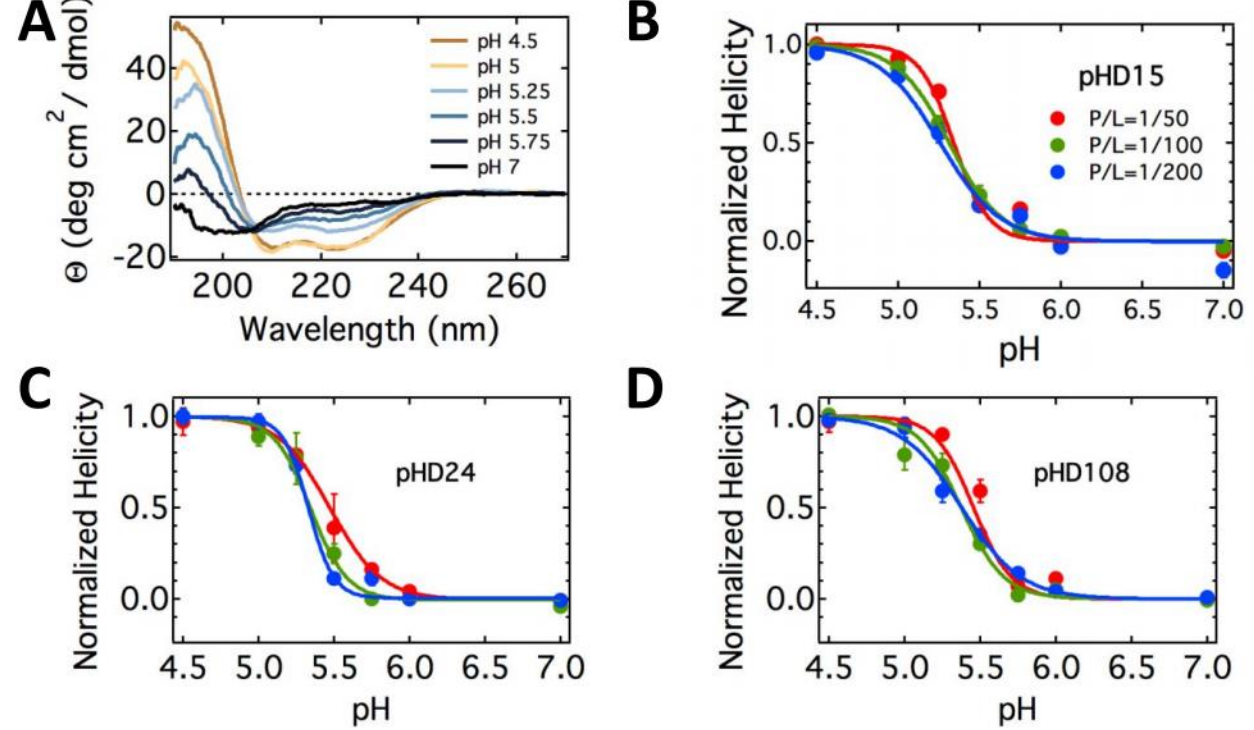

Figure 29 Secondary structure of the pHD peptides, as a function of $\mathrm{pH}$. (A) Circular dichroism spectra of pHD108 at P:L = 1:200, measured after 1 hour of incubation with $1 \mathrm{mM}$ POPC vesicles. Separate samples were made for each $\mathrm{pH}$. (B, C, D) The \% helicity, measured as a function of $\mathrm{pH}$, was calculated from the absorbance at $220 \mathrm{~nm}$, and scaled from 0 and 1 for pHD15 (B), pHD24 (C), and pHD108 (D).

\section{Acquisition of secondary structure upon membrane binding}

To determine the secondary structure of the peptides, we used circular dichroism (CD) spectroscopy. We could only study peptides at the highest three peptide 
concentrations because the technique lacks high sensitivity. As shown in Figure 29A, the CD spectra of the $\mathrm{pHD}$ peptides transition from a spectrum typical of a random coil with one minimum at $200 \mathrm{~nm}$ at $\mathrm{pH} 7$ to a spectrum characteristic of an $\alpha$-helix with two minima at 208 and $220 \mathrm{~nm}$ at $\mathrm{pH}$ 4.5. The spectra indicate that the $\mathrm{pHD}$ peptides undergo transitions from random coil to $\alpha$-helix as $\mathrm{pH}$ is decreased from 7 to 4.5. In Figure 29B$\mathrm{D}$, we show the relative $\%$ helicity as a function of $\mathrm{pH}$ and P:L for pHD15, pHD24, and pHD108. Folding, like binding and leakage, is dependent on $\mathrm{pH}$ and exhibits a cooperative transition occurring over $1 \mathrm{pH}$ unit. All peptides show little to no change in $\mathrm{pH} 50$ as peptide concentration is increased over the available range. The $\mathrm{pH} 50$ for pHD15 is $5.3 \pm 0.1$ for all P:L. For pHD24, the pH50 increases from 5.3 to 5.5 , and for pHD108 the pH50 increases from 5.4 to 5.5 as P:L increases from 1:200 to 1:50.
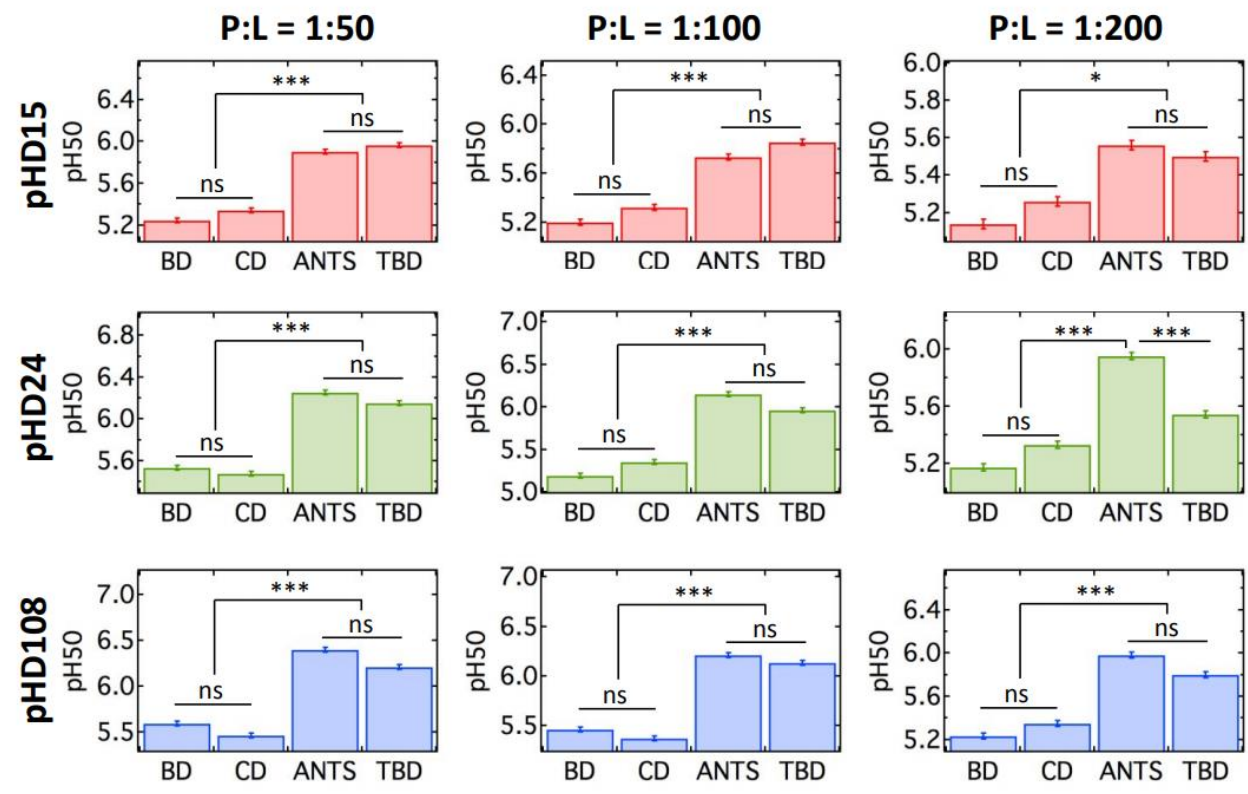

Figure 30 Comparison of pH50s for binding (BD), folding (CD), ANTS/DPX leakage (ANTS) and TAMRA-biotin-dextran leakage (TBD) pKa's. Significance was calculated by ANOVA and a 
Tukey test for multiple comparisons, where $* * * *$ indicates $\mathrm{P}<0.0001, * * *$ indicates $\mathrm{P}=0.0001$ to $0.001, * *$ indicates $P=0.001$ to $0.01, *$ indicates $P=0.01$ to 0.05 , and $n s$ indicates $P \geq 0.05$.

\section{Comparison of pH50 values for binding, folding, and leakage of vesicle} contents

In Figure 30, we compare the $\mathrm{pH}$ dependence of binding, folding, small molecule leakage and macromolecular leakage for the three highest peptide concentrations, for pHD15, pHD24, and pHD108. Several features are common for all peptides, at all P:L ratios. First, the differences in $\mathrm{pH} 50$ values for binding and helicity are not statistically significant, which is expected, given the binding-folding coupling that is typical of membrane active peptides ${ }^{92}$. $\mathrm{pH} 50$ for binding and structure also do not depend strongly on peptide concentration (discussed below). Second, the differences in $\mathrm{pH} 50$ for dextran and ANTS/DPX leakage are not statistically significant, with one exception. Third, and most interesting, the $\mathrm{pH} 50$ values for binding and helicity are always significantly lower than $\mathrm{pH} 50$ for leakage. This effect is most pronounced at high peptide concentrations, a consequence of the observation in Figure 25 and Figure 27 that the $\mathrm{pH} 50$ values for leakage increase with increasing peptide concentration. 

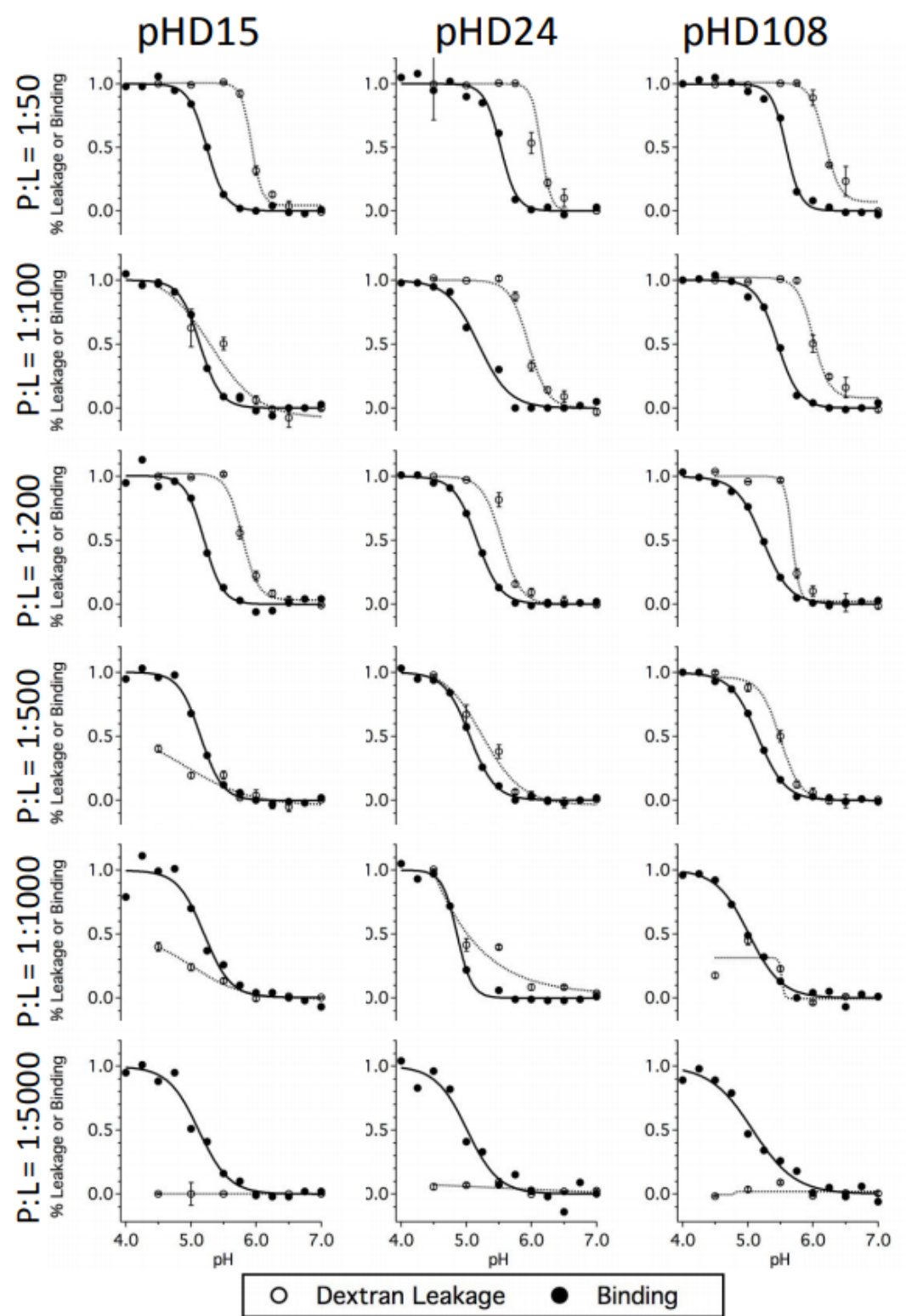

Figure 31 Comparison of binding and dextran leakage activity curves as a function of $\mathrm{pH}$ and P:L. At the highest P:L, $100 \%$ leakage is possible when only a small fraction of peptide is bound, and at the lowest P:L, no leakage occurs even when all peptide is bound.

\section{Comparison of binding and dextran leakage pH50s}


An intriguing consequence of the higher $\mathrm{pH} 50$ of leakage than of binding and folding is that at high overall peptide-to-lipid ratio, leakage is high despite the fact that binding and helicity appear to be negligible. In Figure 31, we compare the binding and dextran leakage curves for pHD15, pHD24, and pHD108 as a function of $\mathrm{pH}$ and peptideto-lipid ratio. At $\mathrm{P}: \mathrm{L}=1: 50$ (top row), the binding and leakage curves are offset such that the $\mathrm{pH} 50$ for binding is lower than that for leakage. At high $\mathrm{P}: \mathrm{L}$, the greatest discrepancies in the fraction bound and fraction leakage occur at intermediate pHs. For example, at $\mathrm{pH} 5.75$, pHD15 induces $96 \%$ TBD leakage when only $3 \%$ of the peptide is bound. At low P:L, the converse is true: leakage is close to zero despite the fact that the fraction bound is high. At $\mathrm{P}: \mathrm{L}=1: 5000$, the $\mathrm{pH} 50$ for binding for $\mathrm{pHD} 15$ is $5.12 \pm 0.05$. The $\mathrm{pH} 50$ for leakage cannot be calculated since no leakage is observed at any $\mathrm{pH}$. The large discrepancies between fraction bound and fraction leakage indicate that the fractional leakage is not dependent on the fraction of peptide bound or folded. 

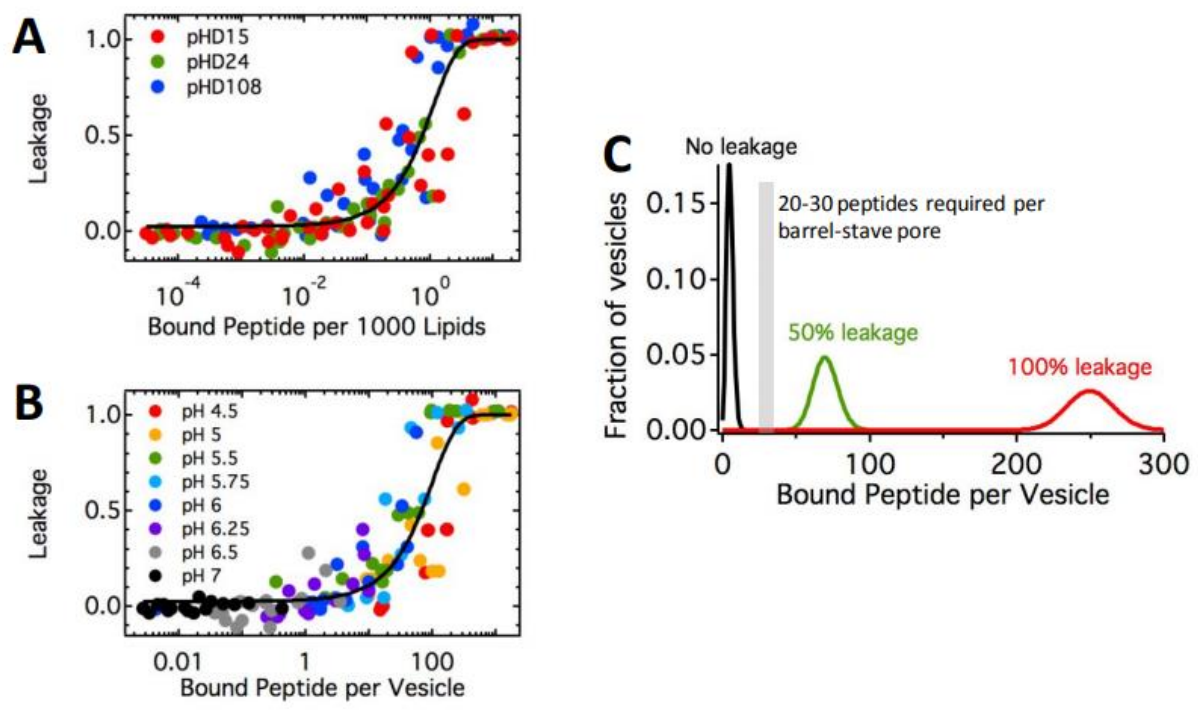

Figure 32 The activity of the pHD peptides depends on the number of bound peptides per vesicle. (A) Dextran leakage as a function of bound peptide per lipid, for the three peptides. Red: pHD15; Green: pHD24; Blue: pHD108. 50\% leakage occurs when roughly 1 peptide is bound per 1300 lipids. (B). Same as in (A), but replotted as a function of bound peptides per vesicle, assuming that each vesicle has $\sim 90,000$ lipids. The different colors indicate the different pHs used in the experiment. 50\% leakage occurs when on average 70 peptides are bound per vesicle. (C). Poisson distribution of peptides per vesicle, when averages are 10, 70,

and 250 peptides per vesicle. Data in (B) show that in these three cases we observe, respectively: no leakage, $50 \%$ leakage, and $100 \%$ leakage. This result can be rationalized, as we calculate that 20-30 peptides are needed to form a barrel-stave pore of $\sim 4.5 \mathrm{~nm}$ diameter to allow for the passage of $\mathbf{4 0} \mathrm{kDa}$ dextran. Thus, no leakage is expected when fewer than 20 peptides are bound per vesicles. Only partial leakage is expected when $~ 70$ peptides are bound, as the pore size distribution in Figure $\mathbf{2}$ favors pores that are smaller than $\mathbf{5} \mathbf{n m}$ in diameter. 100\% leakage is expected when more than 200 peptides are bound per vesicle.

\section{Leakage is dependent on the number of peptides bound per vesicle}

We sought to explain the discrepancies between fractional leakage and fraction bound by accounting for the actual amount of peptide that is bound to membranes under 
the different conditions. Since only bound peptides can induce pore formation, we calculated the number of peptides that are bound per lipid, under each condition, to determine if leakage scales with this variable. In Figure 32A, we show leakage as a function of bound peptide per lipid for pHD15 (red), pHD24 (green), and pHD108 (blue). Plotted in this way, all of the data for all three $\mathrm{pHD}$ peptides can be fitted by a single sigmoidal curve (solid black line), indicating that the pHD peptide function depends solely on the amount of bound peptide. Leakage of $50 \%$ occurs when 1 peptide is bound per 1,300 lipids, a manifestation of the exquisite potency of the $\mathrm{pHD}$ peptides as we know of no other peptide, except the closely related macrolittins ${ }^{22}$, that approach this potency for macromolecular poration.

Assuming a $100 \mathrm{nM}$ diameter vesicle, and an area per lipid of $70 \AA ̊ 2$, we calculate that there are about 90,000 lipids per vesicle. In Figure 32B, we rescale the data in Figure 32A, now plotting the leakage as a function of bound peptides per vesicle. The different colors in Figure 32B indicate the different $\mathrm{pHs}$, and provide an illustration of the fact that the $\mathrm{pH}$ controls function entirely by controlling the amount of bound peptide. In Figure $32 \mathrm{~B}$ we further see that $50 \%$ leakage occurs when only 70 peptides are bound per vesicle.

To rationalize these data, in Figure $32 \mathrm{C}$ we plot the Poisson distribution of peptides per vesicle, when averages are 10, 70, and 250 peptides per vesicle, corresponding to the cases of no leakage, 50\% leakage, and 100\% leakage (according to Figure 32B). We further estimate the number of peptides that are required to line a pore that permits passage of a $40 \mathrm{kD}$ dextran through the bilayer. In particular, we reason that the pore must have a radius that is at least equal to the hydrodynamic radius of $40 \mathrm{kDa}$ 
dextran, $4.5 \mathrm{~nm}$. If we assume that the peptides are in contact with each other and that the diameter of the pHD helix, with side chains included, is $1.2 \mathrm{~nm}$, we calculate that $20-30$ peptides are required to line the perimeter of such a pore. This is called a barrel-stave pore $^{93}$. Thus, little macromolecule leakage can be expected when fewer than 20 peptides are bound per vesicle, consistent with the experiment. At least partial leakage becomes possible once the 20-30 peptide cut-off is exceeded. It is also possible that the peptides form toroidal pores, lined by both peptides and lipid headgroups ${ }^{93}$. In this case, fewer peptides would be required to line the pore. On the other hand, some peptides could line non-productive pores with diameters that are too small to allow the passage the $40 \mathrm{kDa}$ dextran. Indeed, the AFM data in Figure 26A\&E show that the most probable pore radius is about $3 \mathrm{~nm}$, and is thus too small for dextran passage. Overall, the partial leakage that is observed experimentally at $<100$ peptides per vesicle is consistent with most of the vesicle-bound peptides participating in one or a few pores.
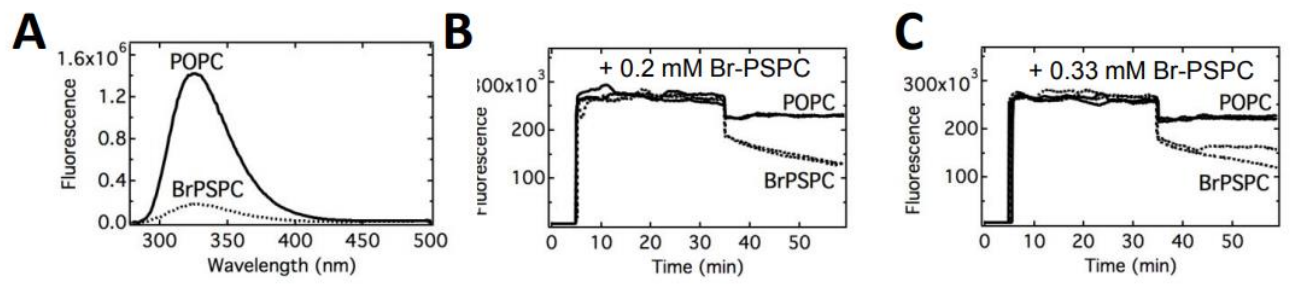

Figure 33 Quenching of tryptophan fluorescence by redistribution of pHD peptides. (A) pHD15 bound to vesicles composed of BrPSPC displays quenched fluorescence. Peptide at $20 \mu \mathrm{M}$ was incubated with $1 \mathrm{mM}$ vesicles at $\mathrm{pH} 4.5$ for 1 hour before the emission spectra of tryptophan was measured $(\mathrm{ex}=\mathbf{2 7 0}, \mathrm{em}=\mathbf{2 8 0 - 5 0 0} \mathrm{nm})$. Peptides in the presence of vesicles composed of POPC or BrPSPC show a blueshifted fluorescence, indicating the peptide is bound. However, the tryptophan peak, in the presence of brominated lipids, is $87 \%$ lower in intensity. (B) Tryptophan fluorescence as a function of time upon the addition of a second batch of vesicles. Original samples are prepared with $1 \mathrm{mM}$ vesicles. At $\mathrm{t}=500 \mathrm{sec}, \mathrm{pHD} 15$ is added at $20 \mu \mathrm{M}$. 
After about $\mathbf{3 0}$ minutes, $0.2 \mathrm{mM}$ POPC or BrPSPC vesicles are added to the cuvette. (C) Same time course as in (B), but $0.33 \mathrm{mM}$ of new vesicles are added after 30 minutes.

\section{Dynamics of peptide association with membranes}

Since a small fraction of bound peptides can cause substantial leakage, it is possible that the $\mathrm{pHD}$ peptides can exchange between vesicles and induce pores on multiple vesicles while the overall fraction of bound peptide remains low. We tested this possibility by evaluating if membrane-bound pHD15 can transfer to new vesicles composed of the brominated lipid, diBrPSPC. The bromines, located on the 9,10 positions on the acyl chains of the lipids, will quench tryptophan when the peptide is bound to membranes ${ }^{94}$ (Figure 33A). Therefore, exchange from POPC to diBrPSPC will lead to quenching. To test for this possibility, pHD15 was incubated with $1 \mathrm{mM}$ POPC vesicles at $\mathrm{pH} 4.5$, where $\geq 95 \%$ of the peptides are bound (Figure $28 \mathrm{C}$ ). After $0.2 \mathrm{mM}$ of POPC or diBrPC vesicles were added, the tryptophan fluorescence intensity decreased (Figure 33B). The decrease caused by PC vesicles can be explained by dilution, however the rapid and much larger decrease into diBrPC vesicles suggests that peptides, initially bound to POPC vesicles, are rapidly quenched by diBRPC lipids with a halftime of a few seconds. Quenching is greater when more diBrPSPC is added (Figure 33C). Most likely, the observed quenching is due to rapid transfer of peptides between vesicles. However, it is also possible that the peptides mediate the fusion of diBrPC and POPC vesicles. 

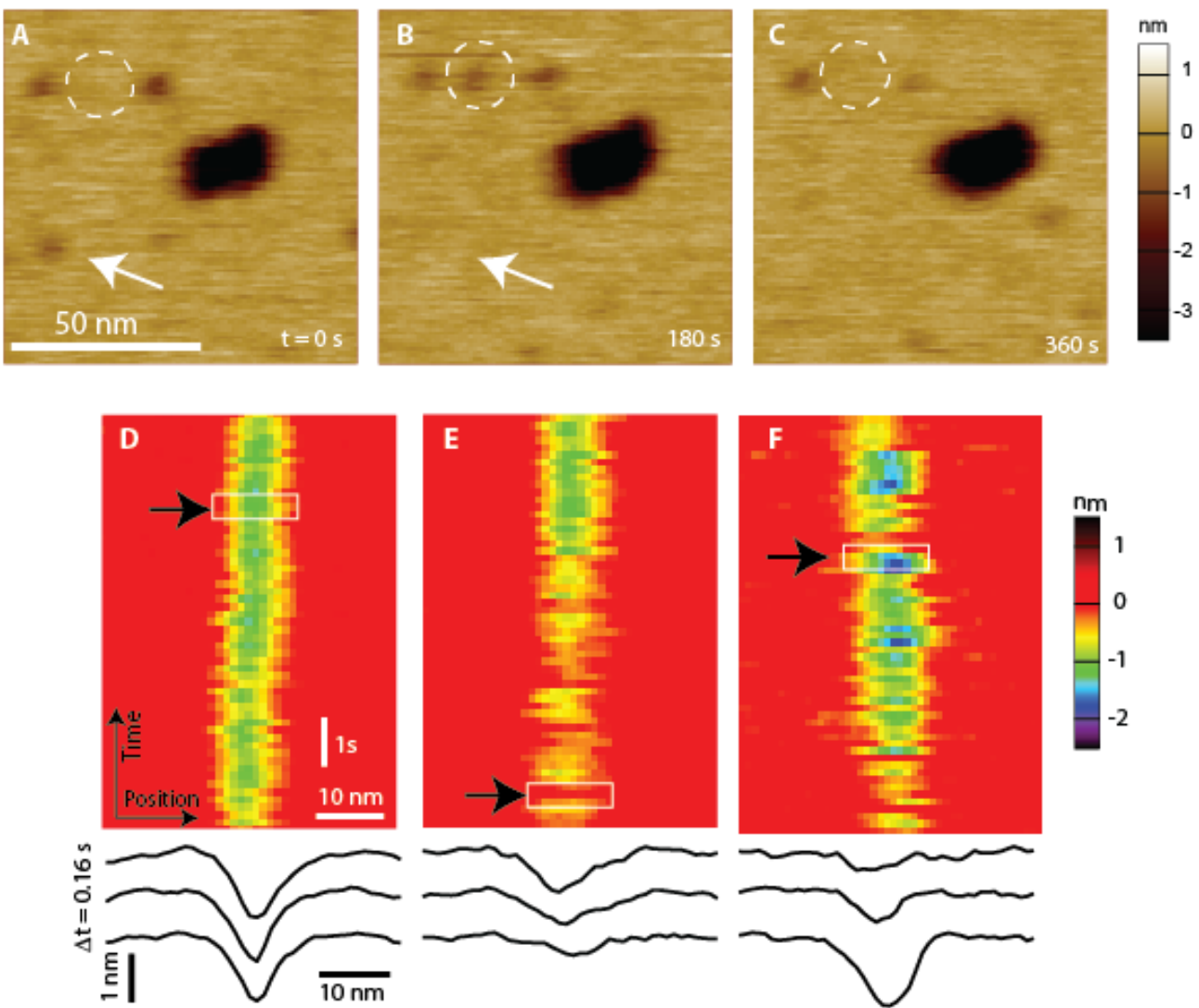

Figure 34 Dynamics of pHD108-induced bilayer topography. (A-C) Time lapse images of a membrane area. White arrows and circles highlight features which appear or disappear on the timescale of image acquisition, red arrows highlight relatively static features. The time interval between images was $180 \mathrm{~s}$. (D-F) Kymographs (time versus position) of pore-like features that were stable (D) or displayed significant temporal dynamics, as evidenced by rapid changes in depth and area ( $E$ and $F$ ). Sets of three sequential line scans are displayed below each kymograph (from the dashed rectangular boxes). The time interval between individual line scans within each set was $0.32 \mathrm{~s}$. All data shown here was acquired at pH 4.

\section{Dynamics of pore formation}

Pore dynamics, if present, can be revealed by repeatedly scanning the AFM tip over the "same" membrane area. Figure 34A-C provides an example. The three images shown were acquired at $3 \mathrm{~min}$ intervals (i.e., $\mathrm{t}=0 \mathrm{~s}, 180 \mathrm{~s}$, and $360 \mathrm{~s}$ ). Two general 
classes of pore-like features can be identified, those which are stable on the timescale of minutes (red arrows) and those which are meta-stable, i.e., can only be visualized in a single image frame (white circles and white arrows) and then, essentially, disappear. For example, the white dashed circle indicates the absence (Figure 34A), presence (Figure 34B), and subsequent absence (Figure 34C) of a pore-like feature at approximately the same location on the membrane surface, as verified by the large stable void in the center right of all images which serves as a reference. In addition, the white arrow indicates a pore-like feature in (Figure 34A) which is absent in the other image frames.

To provide a more detailed view of pore dynamics, we acquired kymograph data (Figure 34D-F). Here, the slow axis of the AFM raster scan was disabled, allowing the tip to scan back and forth laterally over (approximately) the same portion of the membrane surface. The resulting time versus position plots reinforce the bimodal behavior identified in the regular images. A kymograph of a relatively static pore like feature is shown in Figure 34D. Over this $\sim 18 \mathrm{~s}$ time course, the topographic distortion of the membrane induced by the peptide was relatively stable, measuring about $1 \mathrm{~nm}$ deep over the entire trace. In contrast, the other two kymographs (Figure 34E,F) display rich and varied dynamics characterized by significant saltatory changes on sub second timescales. Differences in conformation and conformational dynamics are also evident in the triplets of line scans shown below each kymograph. Each line scan within a set (located by the black dashed rectangles superimposed on the kymographs) are separated temporally by 0.32 s. Clearly, the line scans shown in Figure 34D are indicative of a more stable topographic structure than those in Figure 34E and Figure 34F. Further work will be 
required to quantify the relative populations of stable versus meta-stable feature populations from the AFM data.

\section{Discussion}

Here we have studied membrane interactions, secondary structure, and function of the $\mathrm{pHD}$ peptides across a range of peptide concentrations and $\mathrm{pH}$ values to better understand their sequence-structure function relationships. Overall, the observed dependencies of binding, structure and membrane permeabilization on $\mathrm{pH}$ are sigmoidal with sharp transitions that occur, in most cases, over $\sim 1 \mathrm{pH}$ unit. In comparison, an unaltered protonation equilibrium will be much shallower; e.g. protonation as described by the Henderson-Hasselbalch equation occurs over about $2.6 \mathrm{pH}$ units, for a $5 \%$ to $95 \%$ transition. This suggests that the protonation/deprotonation of the acidic residues occurs synergistically. The pH50 values, ranging from 5.0 to 6.4 for the various measurements reported here, are significantly higher than the inherent $\mathrm{pKa}$ values of isolated Asp and Glu side chains, $(\sim 3.5)$, further implying that there are significant interactions between these side chains and that they are not acting independently. Indeed, the charges engineered into the pHD library are positioned with helical spacings so that they will repel one another maximally when the peptide has $\alpha$-helical structure. Finally, the very fact that the screen selected only for peptides with five or six acidic residues suggests that the synergistic interactions between the charges is critical for $\mathrm{pHD}$ function.

\section{Cooperativity in binding}

For peptide binding, positive cooperativity with peptide concentration will arise if there are strong attractive interactions between peptides in the membranes that are at least 
as energetic as the peptide membrane interactions. This can occur if peptides selfassemble tightly into higher order oligomeric structures in the membrane. While this effect has been reported at least once ${ }^{95}$, it is not frequently observed, probably because membrane partitioning is inherently stronger than the likely magnitude of peptide-peptide interactions in membranes. Alternately, if the presence of peptides in the membrane changes the membrane structure in a way that improves the binding of additional peptides, positive cooperativity could be observed. This scenario could occur, for example, if the expansion of a membrane pore is more energetically favorable (or less costly) than its initial formation of the pore.

For the $\mathrm{pHD}$ peptides, positive cooperativity will result in a rightward shift of the pH-binding curves for higher total peptide concentration. With pHD24 and pHD108, we indeed observed small upward shifts in $\mathrm{pH} 50$ for binding at the highest peptide concentrations, suggesting positive cooperativity (Figure 28). However, given that the peptide concentrations varied by 100 -fold, the cooperativity is small. The binding of one peptide to the bilayer is not strongly influenced by the presence of other peptides already bound to the membrane. Peptide-membrane interactions are dominant over peptidepeptide interactions. Thus, we can describe pHD peptide binding and folding in classical thermodynamic terms of membrane partitioning ${ }^{96-98}$.

\section{Coupling of binding and folding}

The actions of peptides that bind to membranes are usually considered within the framework of a canonical thermodynamic model that includes coupled partitioning of the peptide into the membrane and acquisition of secondary structure ${ }^{97,99}$. This well- 
supported concept of binding-folding coupling is a consequence of the high energetic penalty of partitioning open peptide bonds into the membrane, relative to a peptide bond that is involved in hydrogen bonded secondary structure ${ }^{99}$. For the pHD peptides the observable properties are intentionally coupled to the protonation states of the 5 or 6 acidic residues that were identified in the high-throughput screen ${ }^{23}$. Potential acidic residues in the library were placed with helical spacing patterns to maximize their influence on binding and structure propensity. Here, we show that binding and folding have very similar dependencies on peptide concentrations and $\mathrm{pH}$, at least for the conditions under which both could be measured. We observe a small positive cooperativity in binding that is not apparent in secondary structure measurements, but the latter is a less sensitive measurement overall and can only be measured for the highest few peptide concentrations. In any event, the pH50 values for binding and folding are not significantly different from one another (Figure 30). They are coupled; binding does not occur without folding and folding does not occur without binding. The steepness of the $\mathrm{pH}$ curves and the upward shift in $\mathrm{pH} 50$ compared to free carboxylate groups shows that there is also coupling of protonation to binding and secondary structure. The fully deprotonated peptides do not interact with membranes or fold into $\alpha$-helices, while protonation of at least some acidic sidechains enables membrane interaction and folding.

\section{The $\mathrm{pH}$ - and concentration dependence of permeabilization}

We also measured the dependence of function, membrane permeabilization to macromolecules, on $\mathrm{pH}$ and peptide concentration. The behavior of function is very different from binding and structure. In this case, the $\mathrm{pH} 50$ values and slopes vary significantly with total peptide concentration. At the highest peptide concentrations, the 
pH50 values for leakage are highest, and the $\mathrm{pH}$ curves are steepest. As peptide concentration is decreased, pH50 and slope both decrease. However, slopes, when measurable, are never as shallow as predicted for simple protonation equilibrium, and pH50 values are never as low as expected for a water-exposed carboxyl sidechain.

For comparison, we also measured the pH50 values for leakage of the small molecule ANTS at several peptide concentrations. The pH50 values for ANTS leakage and for dextran leakage are statistically indistinguishable from one another. This demonstrates that pore formation for small molecules and for macromolecules are not distinct processes. Small molecule leakage occurs at lower Pbound:L because there is a distribution of pore sizes (Figure 26), only some of which can pass macromolecules, but smaller and larger pores form with the same pH50.

\section{Coupling of binding and poration}

Interestingly, the functional data show that efficient poration can occur at $\mathrm{pH}$ values significantly higher than those that enable efficient binding, an effect that becomes greater for higher peptide concentrations (Figure 31). Indeed, there are $\mathrm{pH}$ values at which substantial macromolecule leakage occurs despite the fact that fractional binding and folding are very small. There are also conditions where there is no leakage despite 100\% binding (Figure 31, bottom row). It might seem paradoxical that binding/folding are not tightly coupled to function (poration). However this paradox is resolved when we consider the dependence of vesicle permeabilization on the peptide bound per lipid, rather than total peptide per lipid (Figure 32). When the functional data are plotted as a function of Pbound:L (i.e. the actual concentration of peptide acting on the membranes), 
the curves, slopes and pH50 values for the various concentrations become similar for all three peptides under all conditions. Leakage depends primarily on how many peptides are bound per vesicle, and thus binding and poration are in fact tightly correlated. The apparent paradox occurs because a small fractional binding can drive efficient permeabilization under conditions when Ptotal:L is much higher than the Pbound:L required for permeabilization.

It is interesting to note that the poration curves as functions of Pbound:L behave as single function despite data that was collected between $\mathrm{pH} 4.5$ and $\mathrm{pH} 6.5$. We conclude from this observation that the structure of the pore is not sensitive to $\mathrm{pH}$ in this range. As long as the protonation state enables some $\mathrm{pHD}$ peptide to bind, the pore can be formed.

\section{Potency, or the number of peptides required to permeabilize a vesicle.}

The plot of macromolecule leakage versus Pbound:L in Figure 8A shows that the concentration that drives $50 \%$ permeabilization of PC vesicles to $40 \mathrm{kDa}$ dextran is around Pbound:L = 1:1300, under all conditions. This is equivalent to 70 peptides per vesicle. The only other membrane permeabilizing peptides known that have this degree of potency for releasing macromolecules from PC vesicles are the closely related macrolittins ${ }^{22}$, which were selected from the same library.

Assuming random dispersal of peptides, a Poisson function will describe the distributions of the number of peptides per vesicle (Figure 32C). Based on the calculation that 20-30 peptides are needed to form a barrel-stave pore of $\sim 4.5 \mathrm{~nm}$ diameter to allow for the passage of $40 \mathrm{kDa}$ dextran ( $4.5 \mathrm{~nm}$ hydrodynamic radius), we can expect no 
leakage when fewer than 20 peptides are bound per vesicles, and indeed we observe no leakage in this case. Only partial leakage can be expected when $\sim 70(\sim 80)$ peptides are bound, as the pore size distribution shown in Figure 26 favors pores that are smaller than the hydrodynamic radius of the dextran probe. Consistent with this expectation, experimentally we observe 50\% leakage when 70 peptides are bound per vesicle (Figure 32B). We observe $100 \%$ leakage once about 200 peptides are bound to each vesicle.

\section{A possible pore structure}

Taken together, the highly potent macromolecular leakage and the large pores observed by AFM, suggest a structural hypothesis for the pHD peptides. At low $\mathrm{pH}$, when at least some of the acidic groups are protonated, the pHD peptides bind to membranes and fold into amphipathic $\alpha$-helices. We hypothesize that these amphipathic helices stabilize exposed bilayer edges, enabling the otherwise energetically unfavorable formation of large open pores. We envision that the amphipathic helices lie at the bilayer edge, exposing charged and polar groups to the aqueous phase while exposing the hydrophobic surfaces of the helices to the bilayer. Perhaps the pore edges are further stabilized by lateral electrostatic interactions between acidic sidechains and the two basic residues on the $\mathrm{pHD}$ peptides.

Our recent description of the macrolittins ${ }^{22}$, closely related peptides that also have very potent macromolecular poration activity at $\mathrm{pH} 7$, supports these conclusions. The macrolittins were selected from the same library as the pHD peptides, except that they were selected for their ability to permeabilize bilayers to macromolecules at $\mathrm{pH}$ 7. They are nearly identical to the pHD peptides in sequence, structure and potency, except that 
they have three acidic residues total, compared to five or six in the $\mathrm{pHD}$ peptides. In the pHD peptides, protonation of several residues in the other acidic positions enables the pore structure to form only at acidic $\mathrm{pH}$, while in the macrolittins this pore structure is possible at $\mathrm{pH} 7$ because of the smaller number of acidic residues.

By oriented circular dichroism, the parent peptide of the pHD library, MelP5, resides in a mostly membrane spanning, perpendicular orientation ${ }^{61}$. The same is true for the macrolittins ${ }^{22}$. Thus, it is likely that the pHD peptides are also mostly perpendicular to the membrane plane at acidic $\mathrm{pH}$. However it is difficult to study the orientation of the pHD peptides because it is difficult to control or determine the effective $\mathrm{pH}$ in the typical oriented CD sample, a stacked multibilayer system that is hydrated through the vapor phase $^{35,100-101}$. Further, AFM has shown that MelP5 causes membrane thinning in the vicinity of the pores, while the macrolittins and pHD peptides cause pores, but not much membrane thinning ${ }^{21-22}$. Thus, by progressing from MelP5 to the pHD peptides and macrolittins, we have evolved peptides that more effectively span the bilayer "edge" and enable large pores to form in the membrane. AFM, shows the dynamic nature of the pores, as they come in and out of existence on the time scale of minutes (Figure 34).

\section{The effect of sequence}

The physical chemistry and sequence of the pHD peptides affects their potency and $\mathrm{pH}$ dependence. For instance, $\mathrm{pHD} 15$, has a lower potency and a lower $\mathrm{pH} 50$ compared to the other two peptides (Figure 25), suggesting a sequence-dependent effect that may be due to the number of acidic residues; pHD15 is the only pHD peptide with six acidic residues, instead of the usual five. On the other hand, the most potent peptide, pHD108, has 5 glutamic acids, which were significantly overrepresented in the pHD 
sequences, whereas pHD15 and pHD24 have 3 and 2 aspartic acids, respectively. These observations of sequence specific effects suggest that additional changes in sequence, which can be either rationally engineered or identified via high-throughput screens from peptide libraries, can be used to fine-tune the properties of the $\mathrm{pHD}$ peptides for desired applications. The physical chemical sequence-structure-function relationships we have described here will be useful in the future design and optimization of membrane permeabilizing peptides for specific applications.

\section{Conclusion}

Here we study the mechanism of action of the pHD peptides, which form large pores in POPC bilayers in a pH-dependent manner. The activity of these highly potent peptides is controlled by both $\mathrm{pH}$ and peptide concentration, and ultimately depends on the number of peptides that are bound per vesicle. The physical chemical sequencestructure-function relationships that we described here will be useful in the future design and optimization of membrane permeabilizing peptides for specific applications. Such applications could include the release of endosomal contents for drug delivery upon acidification, as well as anti-cancer therapies that exploit the low $\mathrm{pH}$ of the tumor environment. 


\section{Project Four: AFM Cantilever Modification}

Sigdel, Krishna P., Pittman, Anna E., Matin, Tina R., \& King, Gavin M. (2018) HighResolution AFM-Based Force Spectroscopy. In Nanoscale Imaging. Springer Nature.

\section{Introduction}

Atomic force microscopy (AFM)-based single-molecule force spectroscopy (SMFS) is a powerful established technique used to probe physical properties of biological macromolecules and their interactions ${ }^{102}$. This technique can be used to extract unfolding pathways of individual domains of soluble proteins ${ }^{103-105}$. The method has also been used to study folding/unfolding mechanisms of integral membrane proteins such as bacteriorhodopsin ${ }^{106-109}$ as well as to probe the interaction between polypeptide chains and fluid lipid bilayers ${ }^{10,110-113}$.

AFM-based force spectroscopy experiments are often performed by attaching biomolecules such as a polypeptide chain or DNA on the apex area of an AFM tip via non-specific interactions. Unfortunately, this strategy often yields low throughput of high quality data. Moreover, it is common to discard rupture events that occur very close to the sample surface and to consider such events non-specific ${ }^{103,106}$. This can limit a number of studies, including those involving peptide-lipid bilayer interactions.

Recent developments in AFM technology have made significant improvements in both force precision and temporal resolution. For example, modifications to commercially available cantilevers can routinely yield sub-pN force precision and achieve microsecond temporal response times ${ }^{114-118}$. 
Here I detail the results of one method of modification to a commercially available cantilever using a dual-beam focused ion beam/scanning electron microscope (FIB/SEM). FIB-modified cantilevers exhibit higher force precision over a wider bandwidth, but require significant time and resources to fabricate.

AFM-based force spectroscopy takes place in an aqueous environment at room temperature. While submerged in the liquid, the tip is subjected to Brownian noise due to the motion of the surrounding fluid molecules. These interactions add noise to any measurement made, and limits the precision of the technique. It has been shown that this noise can be reduced if the cross sectional area between the tip and the fluid is reduced ${ }^{119}$. As commercially sold, AFM tips are solid and do not have low cross section options. However, reducing the cross section area of the cantilever is easily achieved by a focused ion beam (FIB). The FIB is able to micromachine materials by accelerating Gallium ions to impinge on the cantilever and remove material. Here, two different cantilever shapes were created by modifying commercially available Biolever Mini (BLAC40TS, Olympus, Japan) cantilevers with a FIB. For both configurations, the cross sectional area was decreased, reducing the drag felt by the cantilever, and ideally allowing the force measurements to reach pico-Newton precision while maintaining high bandwidth (>10 kHz). 


\section{Results}
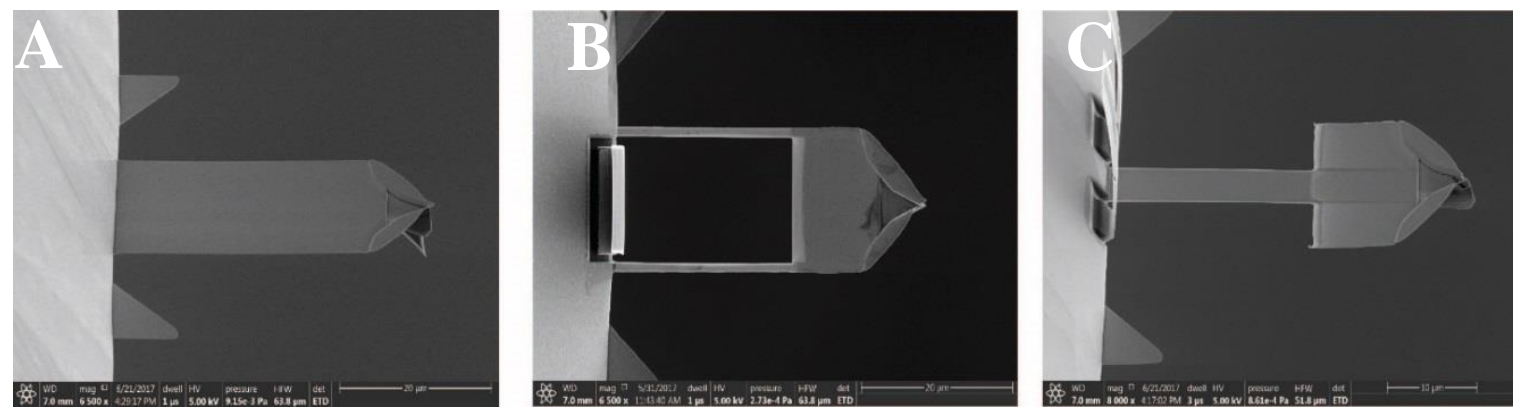

Figure 35 From left to right SEM images of: commercially available BioLever Mini AFM cantilever; configuration one modified tip; configuration two (war-hammer) modified tip.

Commercially available Biolever Mini AFM cantilevers were modified into two configurations using the FIB/SEM. Figure 35A shows an unmodified cantilever, while Figure $35 \mathrm{~B} \& \mathrm{C}$ show the two final configurations. For all images the tip of the cantilever is pointed into the page (the edge of the tip can be seen in Figure 35A).

For the first configuration (Figure 35B) a rectangular section was drilled out of the base of the cantilever. For the second configuration (Figure 35C), called the warhammer, both sides of the cantilever were drilled out, leaving only a central support rod. Additionally, the support rods for both configurations were lightly imaged using the FIB. This had the effect of removing the top gold coating on the support rods. If they had been 'imaged' with the FIB for longer then all of the layers would have been removed, as was done to remove the sections of the cantilever. This removal of the gold coating served two purposes: it has been shown that removing the metallic surface reduces thermal drift $^{120}$, and it allowed the cantilevers to relax down to the horizontal. After the sections were drilled out, both configurations experienced a severe bend above the horizontal due to the imparted stress. The removal of the gold coating helped to alleviate that. Care was 
taken so that the gold coating remained on the broad section of the cantilever directly above the tip, as the laser impinges on that area. Sputtering - that is, Gallium ions which hit outside of the target area-caused some of the gold coating to be removed on the broad section, which led to a loss in signal.

In order to assess the success of the modifications, three major parameters were used: the spring constant, behavior of the harmonic oscillator, and the frequency at which noise was sub-pN. In order to measure the spring constant before and after modification the thermal noise was measured ${ }^{12}$, as detailed in the introduction.

The spring constant of the unmodified cantilever was measured to be roughly 50 $\mathrm{pN} / \mathrm{nm}$. The spring constant of the first configuration (Figure $35 \mathrm{~B}$ ) was $10 \mathrm{pN} / \mathrm{nm}$ and the war-hammer configuration (Figure 35C) had a spring constant of $7 \mathrm{pN} / \mathrm{nm}$. Considering that the spring constant for a cantilever is proportional to width ( $w)$ and thickness $(t)$ and inversely proportional to length $(L)$ (Eq. 7), it was expected that these modifications would lower the spring constant. The benefit of lowering the spring constant is that the low frequency force drift is lower as compared to a stiffer spring.

$$
k \alpha \frac{w t^{3}}{L^{3}}
$$

All modifications serve to lower the drag force felt by the cantilever by reducing the cross-sectional area coming to contact with the room temperature buffer. According to the Fluctuation-Dissipation Theorem (Eq. 8), lowering the hydrodynamic drag, $\beta$, will increase the force precision.

$$
\Delta F=\sqrt{4 k_{B} T \beta \Delta f}
$$

Here $F$ is force, $k_{B}$ is Boltzmann constant, $T$ is temperature, $\beta$ is the hydrodynamic drag, and $\Delta f$ is the bandwidth of the measurement. 
The next parameter, behavior of the harmonic oscillator, is best seen by analyzing the power spectral density (PSD) in Figure 36. The PSD is an auto-correlation function which in effect describes the noise in the cantilever as a function of frequency ${ }^{13}$. For an underdamped harmonic oscillator, the PSD will show a marked rise at the resonance frequency of the oscillator, its 'ringing frequency' so to speak. An ideal cantilever for force spectroscopy would be an overdamped harmonic oscillator. That is to say, the ideal cantilever would experience no ringing regardless of the frequency of data acquisition. As commercially sold, cantilevers are underdamped - a characteristic that is exploited for tapping-mode imaging, where it is necessary to oscillate the cantilever at its resonance frequency. In Figure 36, the black curve shows the PSD for an unmodified cantilever; note the large resonant peak after $10 \mathrm{kHz}$. This peak is less pronounced for both the red (war hammer configuration) and the grey (configuration one) curves.

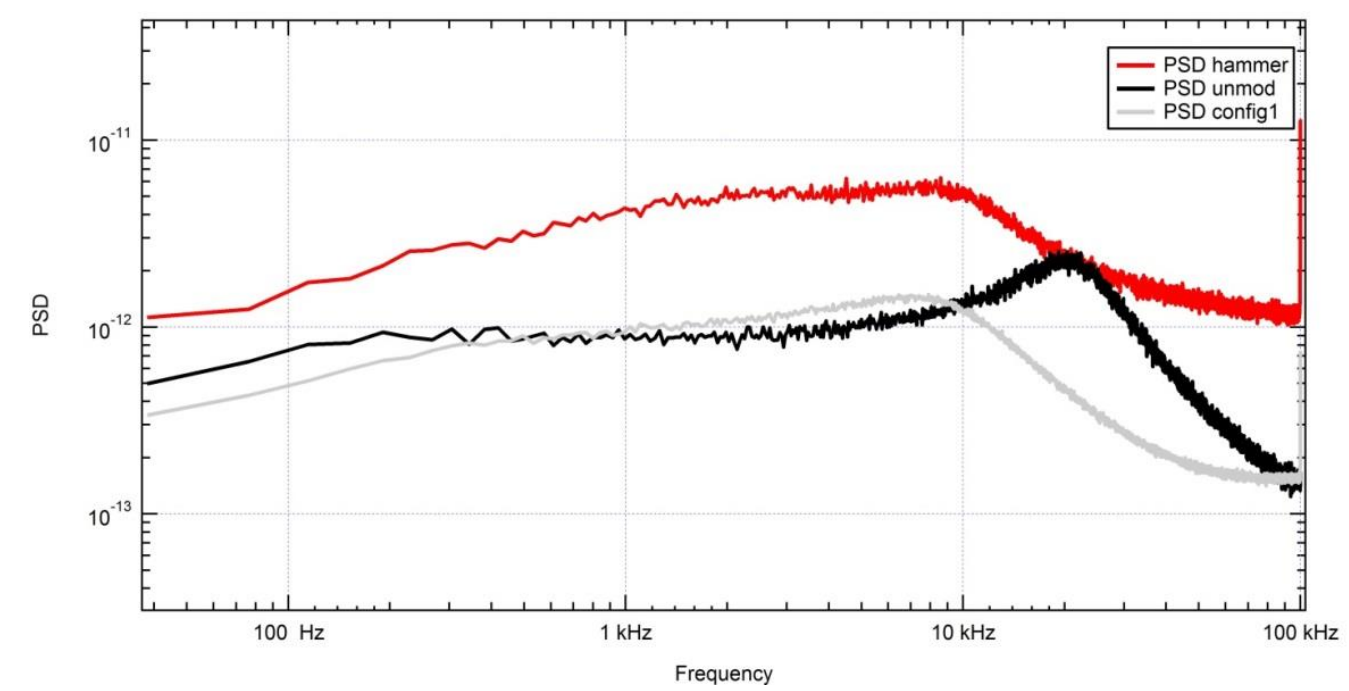

Figure 36 Power spectral density data for the unmodified cantilever (black), the hammer configuration (red), and the first configuration which featured two supporting legs.

The last parameter of interest is the force noise as a function of frequency. By integrating the PSD, one can measure the force noise and ascertain the relationship 
between noise and frequency. Figure 37 shows noise at varying frequencies for both an unmodified (left) and a modified (right) tip. For this figure the unmodified tip used as a Biolever long, but the same trend holds true for the Biolever mini. The FIB-modified tip (here a war hammer configuration) shows sub-pN precision at frequencies above $100 \mathrm{~Hz}$, which is an improvement on the unmodified results.
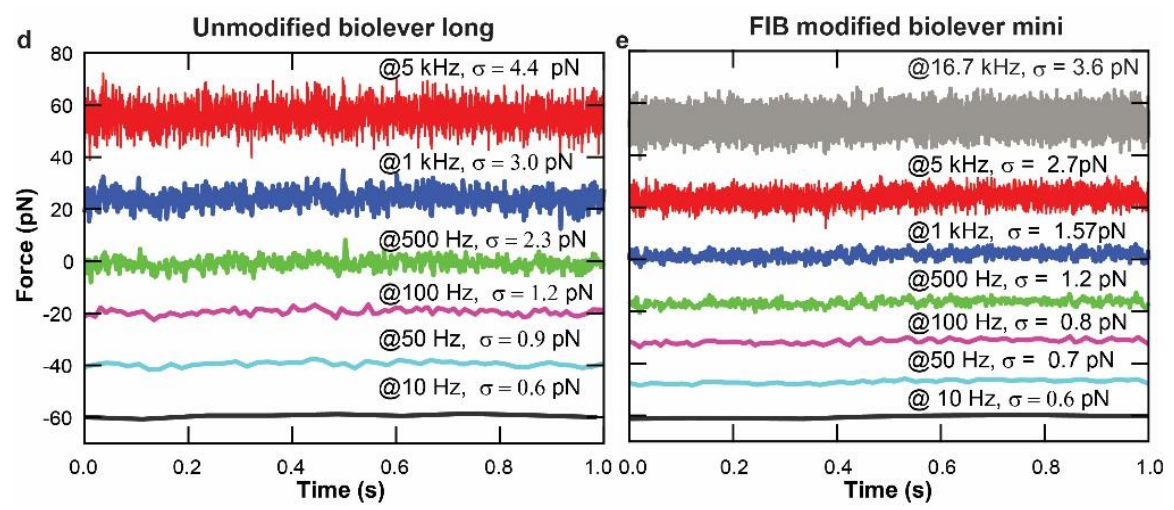

Figure 37 Force precision at various bandwidths for (d) the biolever long cantilever and (e) the FIB modified Biolever Mini cantilever. Note that both tips exhibit similar sub-pN force precision at low frequency: $0.6 \mathrm{pN}$ at $10 \mathrm{~Hz}$; however, the FIB modified tip maintains sub-pN precision at frequencies above $100 \mathrm{~Hz}$.

Figure 38 ties everything together. A war-hammer modified tip (Figure 38A) was functionalized with a five residue peptide and lowered towards a lipid bilayer. Panel B shows the deflection of the tip as it was retracted from the lipid bilayer. The peptide-lipid dissociation rupture event is highlighted with a dashed box. The advantage of the modified tip is seen clearly in panel $\mathrm{C}$, a zoom in of the rupture event. An identical experiment was run with an unmodified tip (blue curve) and then with a modified tip (red curve). It is clear how much more information is garnered by the modified tip, owing to all of the modifications previously detailed. 
a

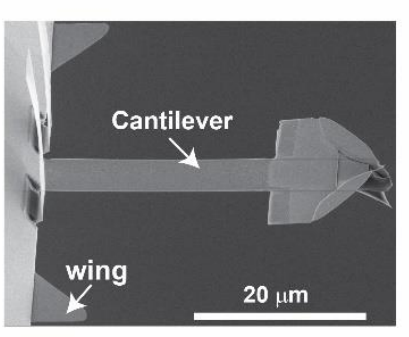

c

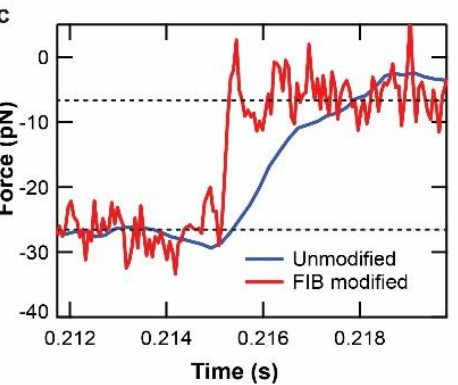

b

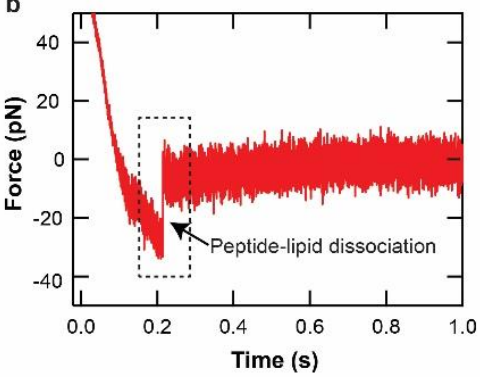

Figure 38 FIB modified tips can achieve higher time resolution without sacrificing force precision. (a) SEM image of a FIB modified Biolever Mini cantilever (as described in Section 3.2). (b) A representative retraction force curve using a FIB modified tip. Here, the tip was functionalized with a peptide (Cys-Trp-Lue-Lue-Lue-Arg) and was interacted with a 1palmitoyl-2-oleoyl-sn-glycero-3-phosphocholine (POPC) bilayer. (c) Zoom in of the curve during dissociation indicates the enhanced time resolution of the FIB modified tip. The blue curve in (c) shows similar data obtained with a non-metalized and non-FIB modified Biolever Long tip (as described in Section 3.1). 


\section{Future Directions}

\section{Planar Bilayer Formation}

Arguably the first step in many AFM experiments is to form a supported planar lipid bilayer. As AFM is primarily a topographical technique, it requires that the sample of interest to be attached to a solid support surface. While this is undeniably an important step in the experiment, it remains poorly understood. The exact mechanics of bilayer formation is disputed, but the general model shown in Figure 39 is agreed upon. The basic steps shown are: absorption of vesicles on the surface, deformation, flattening, and then rupturing to form a continuous supported lipid bilayer ${ }^{121}$.

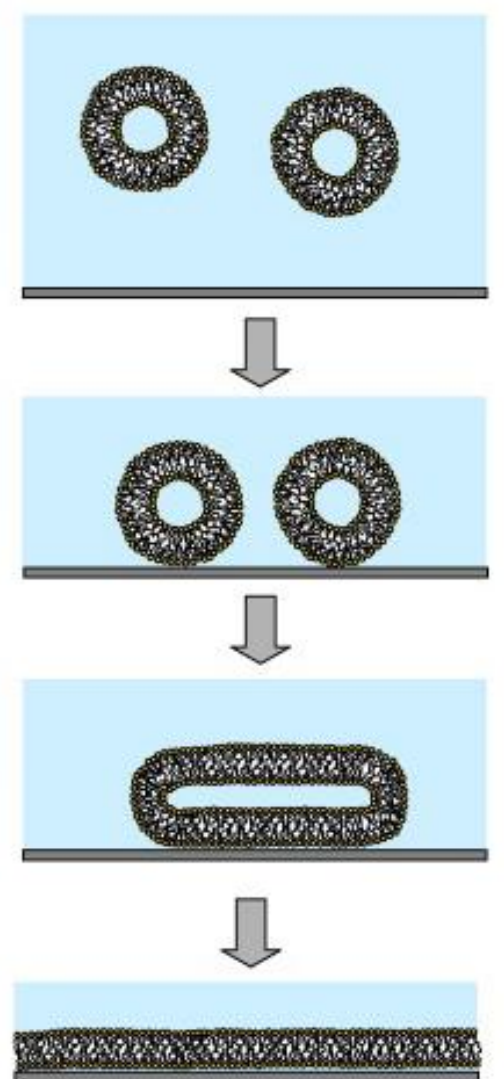

Figure 39 Model for planar bilayer formation ${ }^{121}$. 
Additionally, it is known that there are many factors which can influence any of these steps. The lipid composition could alter the electrostatic interaction between the lipid head group and the solid support (typically mica). The temperature of the solution could alter the temporal dynamics of rupturing. Also, the ionic strength and ionic species of the buffer will have an effect. It is known that the presence of calcium, a divalent salt, has a large influence, and helps to mediate the lipid deposition process ${ }^{122}$. Richter, et al, even propose that the tip-lipid force can be modulated to induce separate membrane patches to merge to a continuous bilayer ${ }^{122}$.

One parameter in particular which is unknown is the temporal dynamics of vesicle rupture. Hamai, et al, monitored deposition of giant unilamellar vesicles (GUVs) using fluorescence microscopy. For their lipids (DOPC, DOPE, DOPG), they observed lipid vesicle rupture $10-20 \mathrm{~ms}$ after deposition on glass ${ }^{123}$.

This rapid timescale does not appear to hold true for conditions used in my peptide-lipid experiments. In my typical peptide experimental setup, the lipid solution is incubated on mica for thirty minutes to facilitate lipid vesicle rupture and bilayer formation. This timing, garnered from numerous trial and error attempts, appears to be sufficient for supported lipid bilayer formation. However, it would be of interest to quantify this bilayer formation in more detail, as there are numerous questions which remain unanswered. Is thirty minutes too long? What is the minimum time required for planar bilayer formation? How does this number change in the presence of peptide?

The method I am proposing to answer these questions is optical microscopy in conjunction with fluorescently labeled POPC. Optical microscopy as a technique allows us to take a step back in length scale as compared to AFM. AFM is a useful technique, 
which can answer many questions, but it is not ideal when investigating large scale (>100 um) effects such as bilayer patches. The mica substrate is not homogenous ${ }^{124}$; thus, different areas of the mica might react differently to the lipid vesicles. Optical microscopy allows for a quick, large scale investigation of lipid behavior.

Preliminary optical microscopy experiments have been run, using POPC with fluorescent tags attached to the lipid tails. Non-fluorescent typical POPC was mixed with the fluorescently tagged POPC so that the final mixture contained $1 \%$ fluorescent POPC. Typical florescent tags are often attached to lipid head groups. However, since the goal is to understand in greater detail the binding of POPC vesicles to the mica substrate, such tags might interfere with the surface chemistry and obscure binding events.

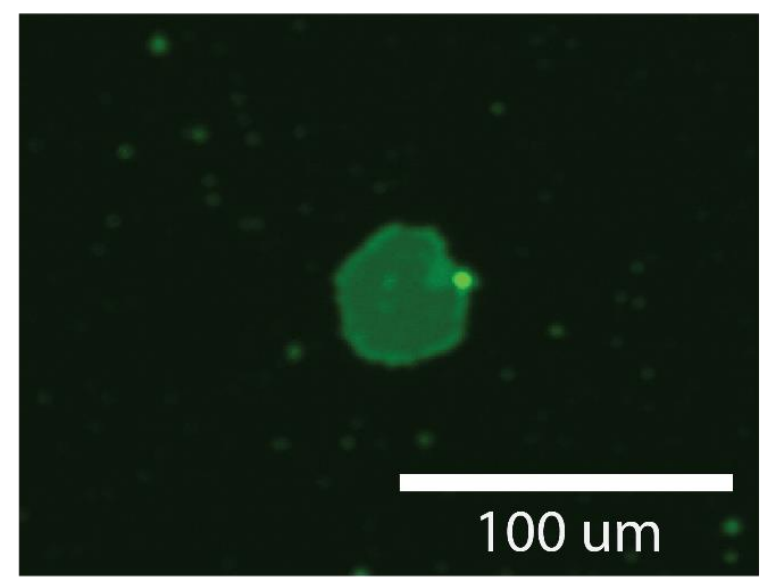

Figure 40. Preliminary results of fluorescently labeled POPC absorbed on a glass slide

Figure 40 shows a representative image of the preliminary results. Here, fluorescently labeled POPC has been absorbed onto a glass slide and imaged in a widefield optical microscope. Mica is difficult, but not impossible, to use in optical microscopy due to its inherent birefringent properties. The experiments will first be optimized for glass — which can be used as a substrate for $\mathrm{AFM}^{44}$ — and then extended to 
mica. In full, the effect of parameters such as time, temperature, ionic strength of buffer, ionic species of buffer, substrate, and peptide concentration on bilayer formation will be better understood.

\section{Lipid Size Effects}

Another parameter which might be advantageous to be fully understood for poreforming experiments is lipid vesicle size. The lipid vesicle size might impact the experiments in one of two ways: it might affect the peptide behavior, and it might affect the planar bilayer formation. It has been shown that certain cell-rupturing peptides exhibit size-dependent behavior ${ }^{125-127}$. Cho et al. investigated a peptide which showed a vesicle size-dependent rupturing behavior ${ }^{125}$. Jackman et al. used that principle to develop a possible Zika virus treatment, by using a peptide that can selectively target small Zika virus particles $^{126}$.

Thus it is possible that lipid vesicle size could impact the action of the poreforming peptides investigated here. This idea occurred after I had much difficulty in reproducing the pHD108 results. The lipids I had been successfully using were about to run out, so I extruded a new batch of POPC lipids. After carefully measuring the concentration of the new batch, I expected to see identical results. However, I was surprised to discover that the pHD108 did not create pores using this new lipid, even though all of the other parameters were held constant. Using the dynamic light scattering machine to size the two lipid batches gave a hint as to a possible reason for the lack of 
pores.

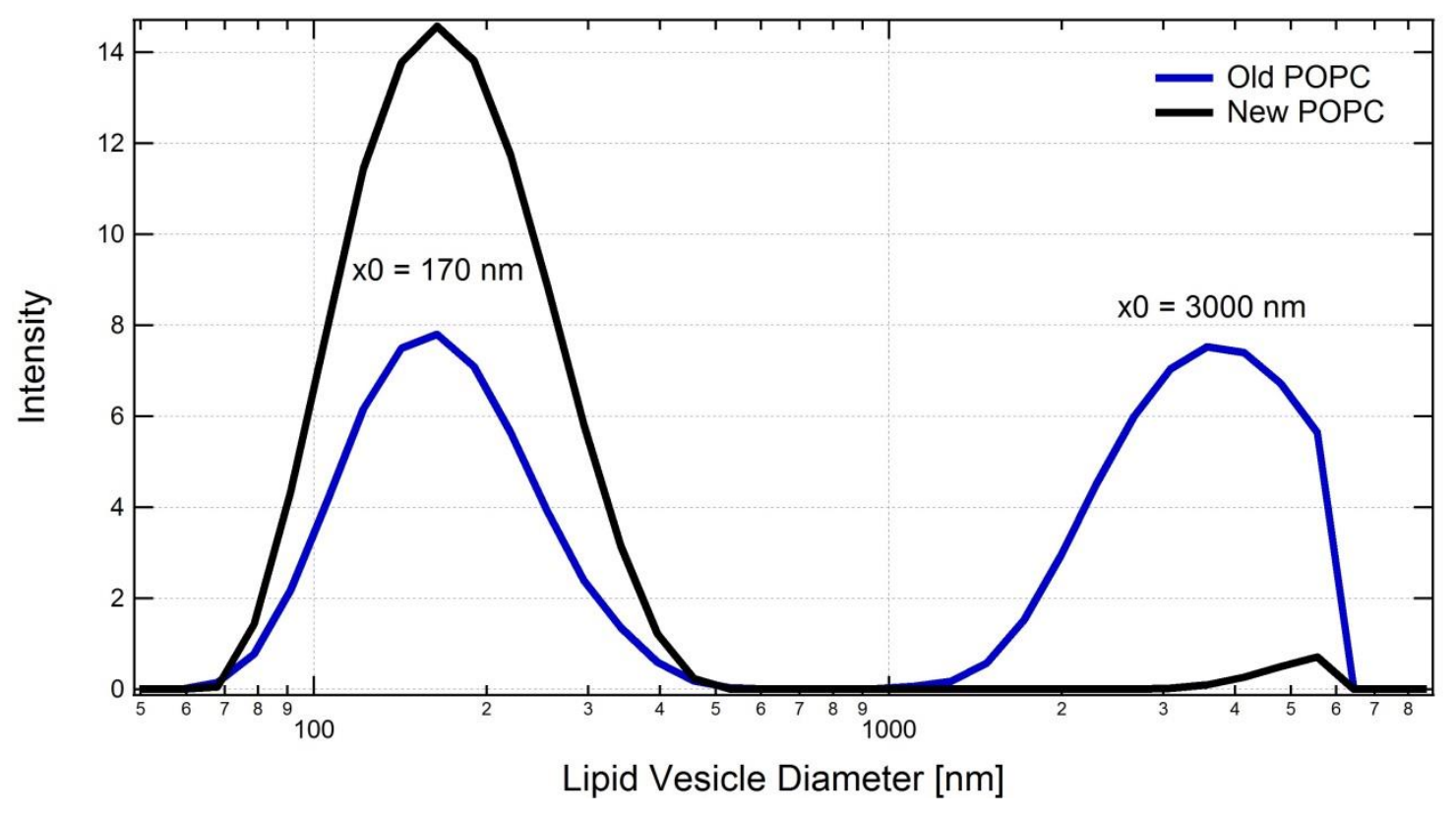

Figure 41. Dynamic light scattering result for two POPC batches

Dynamic light scattering is a tool used to measure the size of small particles. It works by measuring the light scattering off of the particles. The scattered light is used to calculate the intensity auto-correlation function. This auto-correlation function allows for the determination of the size of the particles.

Figure 41 shows the result of the dynamic light scattering. The old batch of POPC — the batch that showed pore-forming behavior with pHD108 — was not comprised of homogenous small vesicles like expected. Rather it showed two prominent populations. Roughly half of the mixture was small ( 100 nm in diameter) vesicles and roughly half were large $(\sim 3000 \mathrm{~nm}$ in diameter). The new batch of POPC (shown in black) was comprised on the expected homogenous population of small lipid vesicles.

This change in lipid vesicle size could be one of the contributing factors to the irreducibility of pHD108. It would be beneficial to study this dependency in further 
detail. Is the pHD108 preferentially binding only to the large vesicles? What would the behavior be like if the lipid mixture was comprised of only large vesicles?

These questions could be answered in conjunction with the planar bilayer formation project detailed above. Once we fully understand how lipid vesicle size effects planar bilayer formation in the absence of peptide, then we can perhaps better interpret the results of lipid vesicle size dependency on pore-formation.

\section{Pore-formation Mechanisms}

How exactly do the pore-forming peptides discussed here actually form pores? One theory of pore formation comes from Huang, et al, who proposed a theory of cooperativity to explain the action of antimicrobial peptides (i.e. melittin) ${ }^{38}$. In this theory, there are two distinct states of the peptides, and the proportion of peptides in each state is a function of peptide to lipid ratio $(\mathrm{P}: \mathrm{L})$. When the $\mathrm{P}: \mathrm{L}$ is below a critical threshold, all of the peptides exist in a surface bound (S) state, which is characterized by a lack of transmembrane pores, the peptide backbones are parallel to the plane of the bilayer, and these peptides induce membrane thinning. As the P:L reaches a critical threshold, the peptides can transition to an inserted (I) state. This state is characterized by transmembrane pores, and the peptide backbones are perpendicular to the plane of the bilayer $^{38}$.

MelP5 appears to be a textbook example of this theory of pore formation. In a typical MelP5 image, there exists areas of thinned bilayer (S state) and in those thinned areas appear pores (I state). Thus, it would appear that the critical P:L mentioned above is extremely localized. It is only when a specific area of the membrane reaches the critical concentration then pores can form. The thinned areas could represent local pools of $S$ 
bound peptides. When that local pool reaches some critical threshold, the peptides can then insert themselves into the bilayer and form transmembrane pores. This would explain why we did not observe MelP5 pores outside of locally thinned portions of the membrane. Pores can only form in areas where enough peptides have locally congregated. Figure 42B is a cartoon representation of this model of pore formation. Note the inserted peptides forming a pore, surrounding by S state peptides.
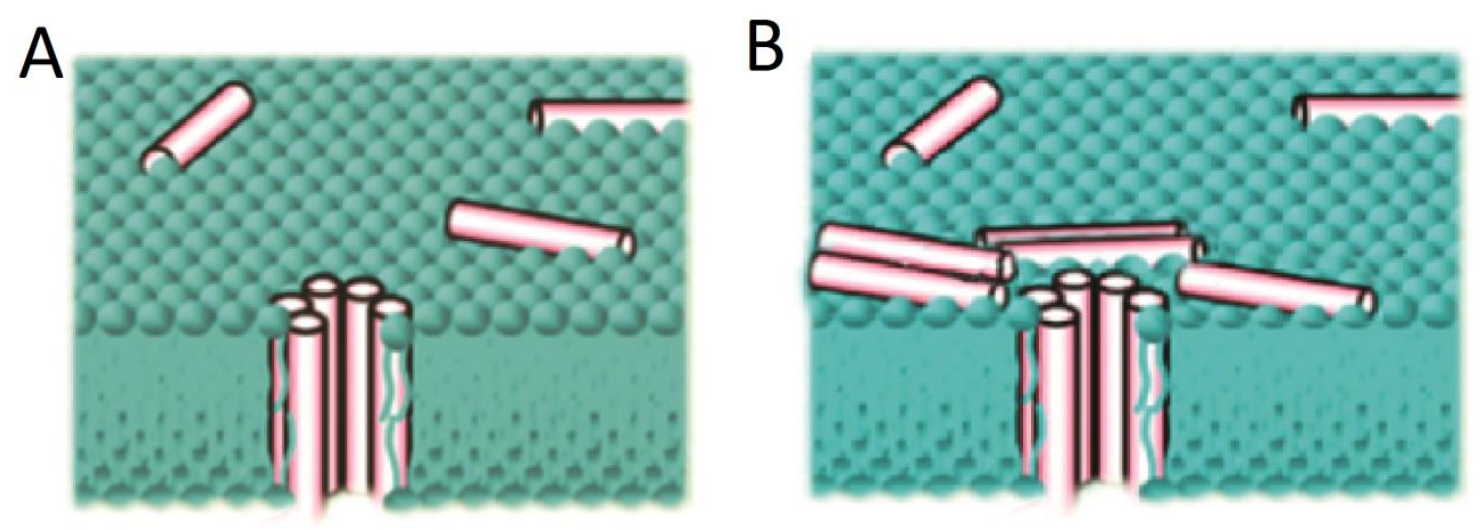

Figure 42. Cartoon representation of pore formation. (A) pores are not surrounded by thinned areas of the membrane and (B) transmembrane pores are surrounded by peptides in the $S$ state, thinning the membrane

This model, while it appears to be relevant to MelP5, does not describe the mechanism for every pore-forming peptide. For example, pHD108 pores do not appear to have any appreciable thinning surrounding them. Thus, this two state model must be reconsidered. Whereas MelP5 peptides oligomerize on the bilayer, perhaps the pHD108 peptides oligomerize in solution. When this oligomer encounters the bilayer it is able to go straight to the inserted state and forms a transmembrane pore. Or instead, perhaps the peptides are oligomerizing on the bilayer, but in a way that does not induce thinning of the bilayer. 
Figure $42 \mathrm{~A}$ shows a possible cartoon representation of this method and also raises questions. Are there any peptides in the S state, as seen in the cartoon? Do the peptides only interact with the membrane after they have oligomerized in solution? Or is it possible that they are bound to the membrane and do not induce any thinning?

Preliminary experiments have attempted to answer those questions. The topography of pHD108 images offers an interesting absence of information. The bilayer appears to be relatively flat around the pore-like features. We can see that there is no appreciable thinning of the membrane caused by the peptides. However, things become interesting when we begin to consider the phase images.

An AFM phase image details the difference between the phase of the driving impulse and the phase of the corresponding tip movement. They can become out of phase depending on the type of material the tip encounters ${ }^{128}$. Thus, the phase image is typically interpreted to show differences in material properties (i.e. elasticity) of a sample. Figure 43 shows a representative pHD108 image with the phase image on the left and the normal height image on the right. 

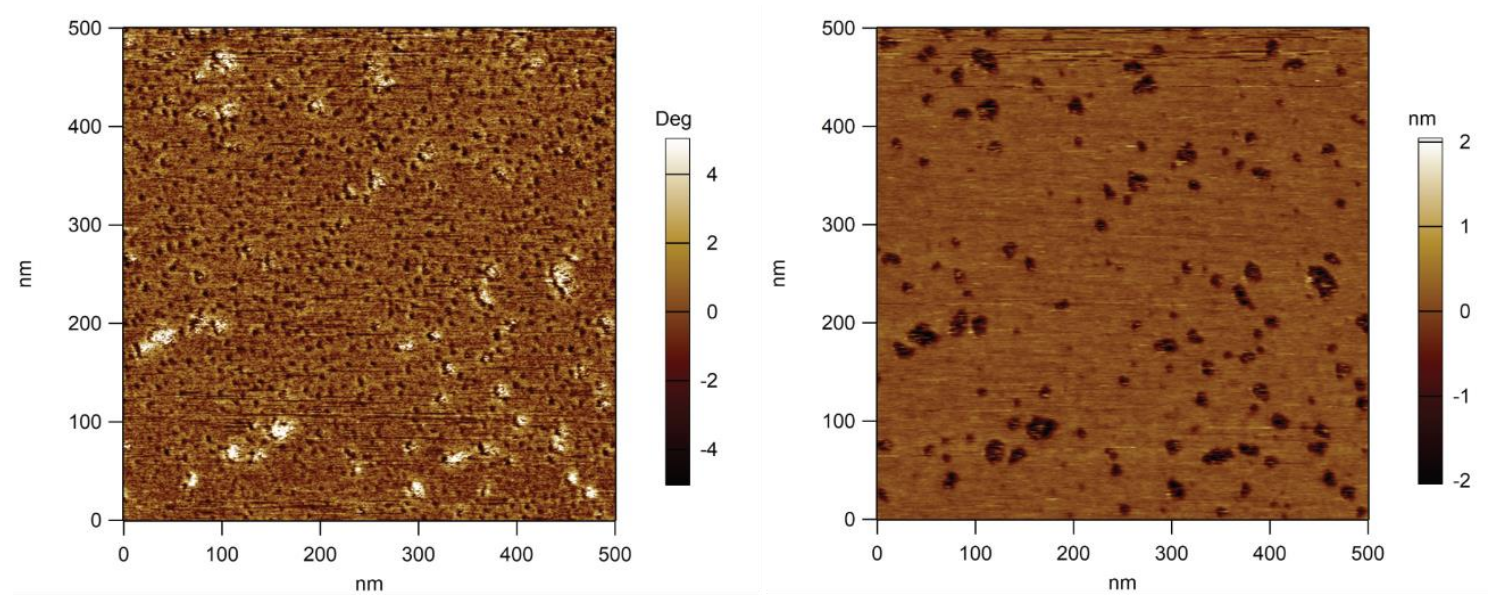

Figure 43. Phase vs height AFM images. Left: phase image of pHD108 induced effects on a lipid bilayer. Right: typical height AFM image of pHD108 induced effects on a lipid bilayer.

As you can see from the right image, there are peptide-induced pores, surrounded by what appears to be a relatively flat bilayer. There does not appear to be thinned regions of the membrane, like there are for MelP5 pores (Figure 13). However, the phase image tells a different story. First, note the light features in the phase image; these correspond to the deep features (pores) in the height image. Next, note the large number of clear, dark, and localized features in the phase image. These do not appear to correspond to any features in the height image. 

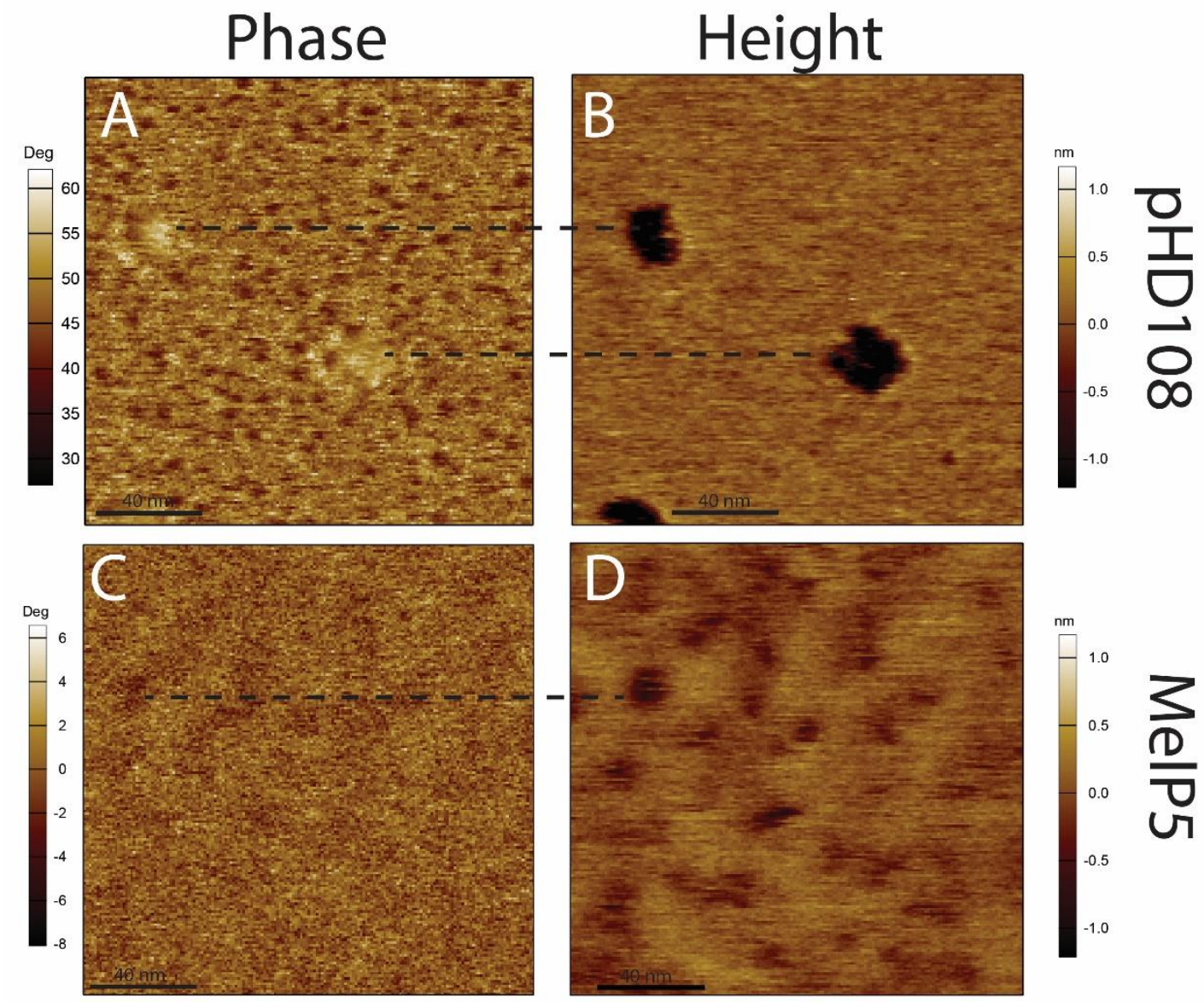

Figure 44. Height and phase images for both pHD108 and MelP5 pores. Dashed lines connect pores in the height and phase images.

Figure 44 shows how the phase data differs for different peptides. Panels B\&D show the topographic images for the pHD108 and MelP5 pores. In panel A the pHD108 pore can be seen in the phase image as interactions with the pore leads to a slightly higher (i.e. lighter) phase. While there isn't anything interesting happening topographically surrounding the pHD108 pores, the phase image shows many small, dark features. MelP5, on the other hand, shows the reverse. The phase data does not show anything interesting happening around the pores, but the topographic image reveals darker (thinned) valleys surrounding the pore (this is more clearly seen in Figure 15). 
The light vs dark color scale of the phase image details a response that either has a leading or lagging phase. Thus the pore-like features and the other features interact with the tip in opposite ways. When the tip encounters a pore, or deep feature, the phase difference has a more positive number (i.e. lighter color in phase image). The tip appears to be encountering something outside of the pores which is causing the phase to have the opposite reaction, as the phase difference is a negative number (darker color in phase image).

Direct interpretation of phase images is difficult. There has been effort to fully characterize the phase data, and Proksch, et al, detailed a method to analyze what they called 'loss tangent imaging' which is intrinsically related to phase imaging ${ }^{129}$. However, their analysis and characterization was done in air, whereas the experiments described here were all performed in fluid.

Stark et al analyzed phase shift images in fluid while using tapping mode AFM to image bacteriorhodopsin ${ }^{130}$. They broke down two major contributions to the phase data: first derivative in scan direction and the geometric contact area between the tip and the sample. That is to say that straight forward interpretation of the phase data is impeded by the fact that two separate factors are contributing: how the topography is changing, and how the contact area between the tip and the sample is changing.

Nagao et al also attempted to quantify phase data from tapping mode AFM in buffer ${ }^{131}$. In their analysis of imaging live cells, they argued that there are two factors contributing to the phase data: stiffness of material and its viscoelastic properties. They didn't consider tip-sample contact to be a large contribution to the overall phase data. 
Additionally, they interpreted a positive phase shift to represent a region that was stiffer and a negative phase shift to represent a region that was less stiff. However they were unable to establish any absolute scale - the only interpretation of the phase data is the relative changes in stiffness ${ }^{131}$. Using this scale, the dark features seen in Figure 43 and Figure 44 would be areas of the membrane which are stiffer than the unaltered lipids.

Regardless, it can be clearly seen that there is something which is not pure lipid which exists outside of the clear pore-like features and that something interacts with the tip differently than the pore-like features do.

One possible explanation is that when the pHD108 peptides interact with the lipid bilayer, they first insert themselves into the membrane (perhaps perpendicular to the plane of the bilayer). These inserted pre-pore peptides do not cause thinning, but they do interact with the tip in a way that is distinguishable from unadulterated lipid.

Future investigation into this phenomenon will hopefully shed more light on the different mechanics of pore-formation.

\section{Concentration Dependent Effects}

As mentioned before, most models of pore formation depend on a critical P:L, below which transmembrane pores are not observed. Investigating pores using AFM lends itself to an even more specific golden P:L. Not only do pores need to exist, they also need to be diffusing slowly on the timescale of imaging. After all, AFM builds up a topographic image by rastering the sharp tip over the lipid surface. This rastering takes time — on the order of minutes. Any pores which are rapidly diffusing over a lipid membrane might appear as single line noise, or might not appear at all on an AFM image. 
One way to ensure that these pores are not rapidly diffusing is to bias the system to a concentration in which many pores are present. This is one explanation as to why the MelP5 pores (Figure 13 and Figure 45A) are clear and stable over the imaging time. Perhaps it is because they lack the room to diffuse, as the lipid bilayer is packed densely with pores. Alternatively, it is also possible that the presence of the negatively charged mica surface below is 'pinning' the positively charged MelP5 pore and thus preventing rapid diffusion.

Regardless, it is clear that the peptide will have differing behaviors dependent on the P:L. Preliminary work has sought to clarify these different behaviors. Figure 45 shows POPC membrane and three different concentrations of MelP5. Panel A is the 1:1200 ratio described in project one of this manuscript. Panel B has an increased concentration of MelP5, and panel $\mathrm{C}$ even more so. It is clear that the changes in peptide concentration have had an increased effect at permeabilizing the membrane. At higher concentrations it appears that the pores have merged and begun to dissolve the bilayer.
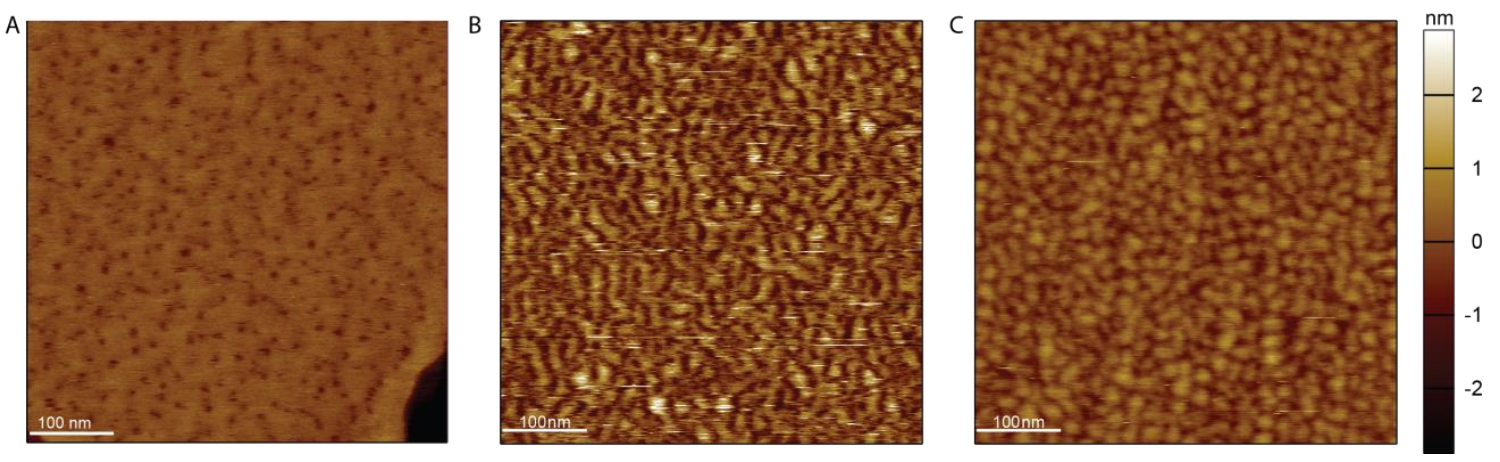

Figure 45. Concentration dependent effects of MelP5. (A) peptide to lipid ratio of 1:1200. (B) 1:200 (C) 1:100

Preliminary work has shown that the converse is true. For pHD108, at lower concentrations the effects are reduced, and the transmembrane pores are few and far 
between (Figure 46). As stated previously, this could be due to a lower density of features leading to a higher diffusion rate.
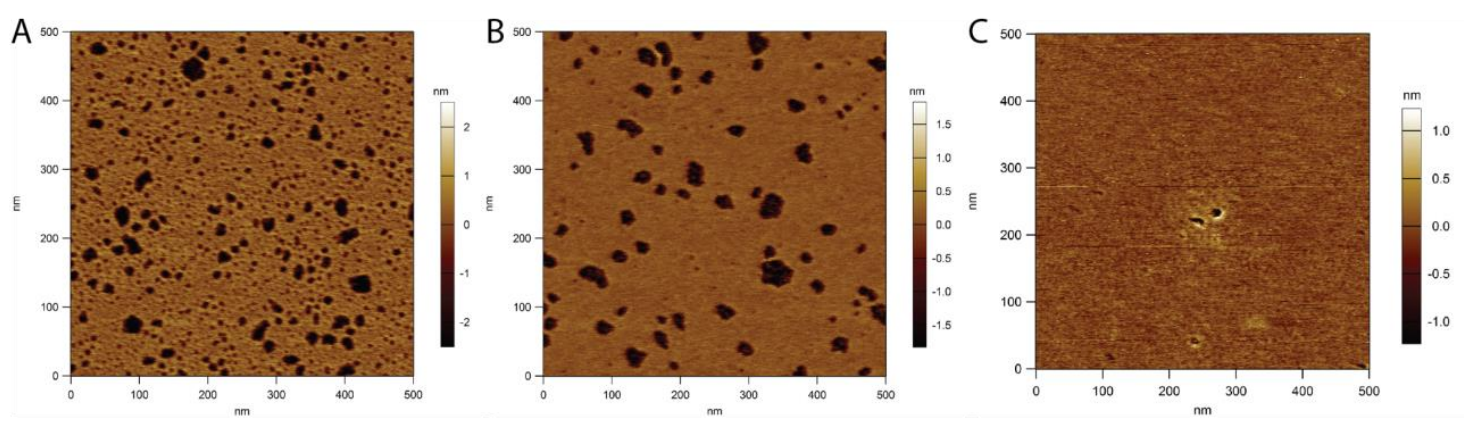

Figure 46. Concentration dependent effects of pHD108. (A) 1:100 uM (B) 1:200 uM and (C) 1:1000

Future experiments can help to answer these questions. According to the theory of cooperativity used to explain the mechanism of MelP5 pore formation, a lower P:L could lead to only thinned areas with no transmembrane pores. Or, perhaps, even at lower P:L there would still be thinned areas with pores, but at a lower density than is currently seen (Figure 13A).

Additionally, the behavior of pHD108 at lower concentrations needs to be studied in greater detail. Can rapidly diffusing pores be detected at low concentrations or are there really just few, static pores? Future work is needed to understand in greater detail how peptide behavior changes as a function of concentration.

\section{Measuring the peptide to lipid ratio}

Before a detailed concentration study, as described above, can be accomplished, the exact peptide to lipid ratio on the surface of the mica must be determined. In the experiments described in this manuscript, an approximate peptide to lipid ratio was 
known. However, recent experiments have shown that these peptide to lipid ratio estimates are most likely higher (that is, over-estimating the amount of peptide) than the exact ratio.

Several factors complicate the true P:L for AFM experiments, chief among them the rinsing step. Before an image is taken, the solution is washed four times to remove any non-ruptured or loosely bound lipid vesicles which could impede clear imaging. It is assumed that any lipid/peptide complex bound to the surface of the mica will not be significantly affected by this rinsing step and that the original P:L will remain constant on the surface. Alternatively, the washing could remove the bound complex, but with the assumption that the peptide and the lipid will be removed concurrently.

This assumption has been called into question with preliminary results from Dr. William Wimley, a collaborator of ours who helped to develop the pore-forming peptides studied here. He ran a mock AFM experiment with pHD108 and POPC vesicles. After depositing the mixture on the mica surface and allowing it to incubate, he washed the sample three times, and measured the amount of peptide and lipid that was coming off with each wash. To achieve this, he used dye labeled POPC and made use of the fact that 
pHD108 has a tryptophan in its sequence.

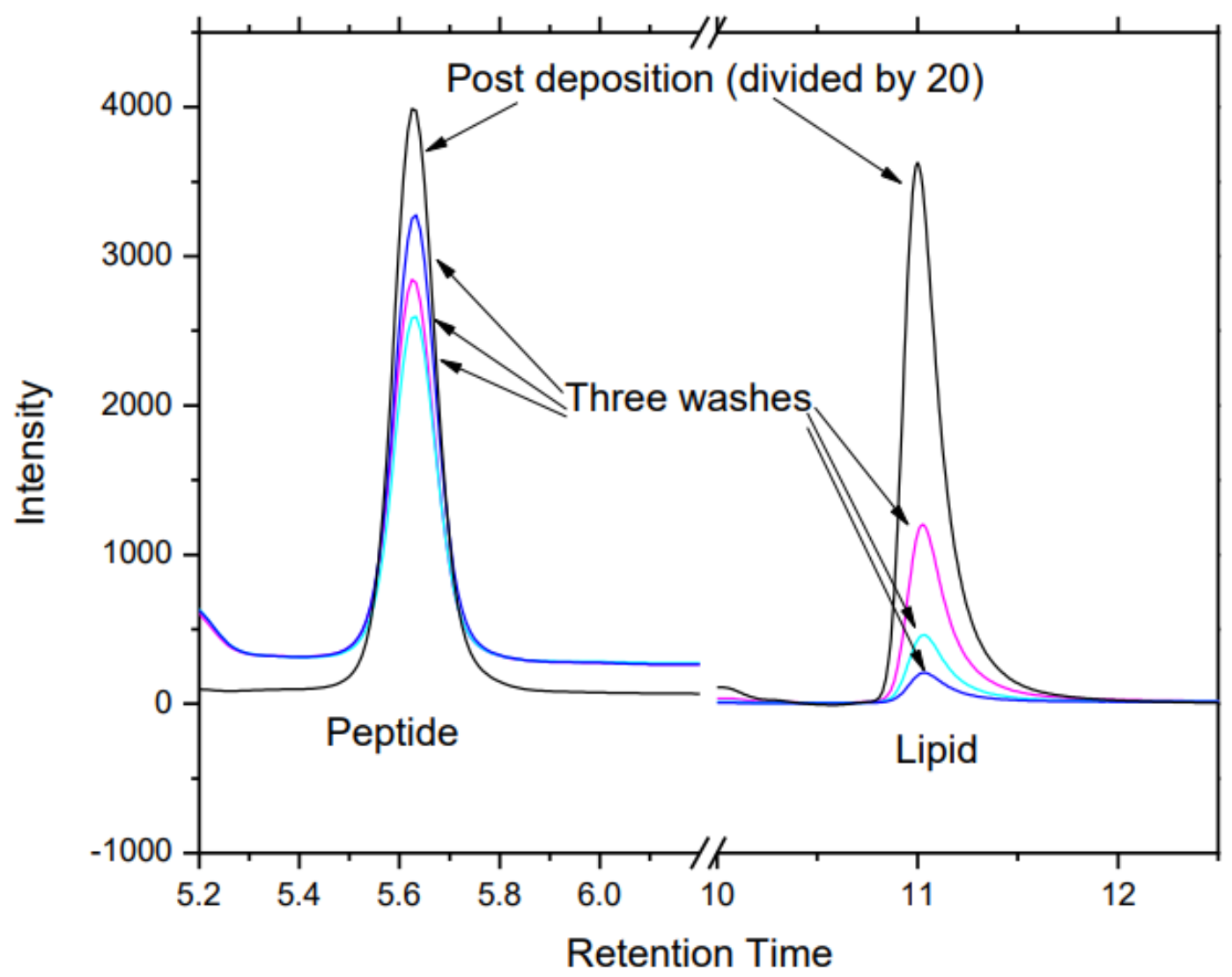

Figure 47. Results of the wash after passing through high-performance liquid chromatography and a UV-Vis detector

Figure 47 shows the results. The black line represents the solution pre-washing.

For each wash, the amount of peptide and lipid being rinsed off was quantified. For the first wash, both peptide and lipid were removed, with more peptide being removed than lipid. However, in subsequent washes, the peptide continued to be removed in large amounts, while fewer lipids were removed each time.

These preliminary results show that our original assumptions about washing were incorrect. First, there appears to be a non-negligible amount of both peptide and lipid being removed. If they both being washed off by the same amount, then the P:L would 
remain the same. However, it appears that the peptide is being preferentially washed off, which would result in a true P:L which is much lower than the assumed P:L. Future work is needed to accurately determine the final P:L. 


\section{References}

1. Ashkin, A.; Dziedzic, J. M.; Bjorkholm, J. E.; Chu, S., Observation of a single-beam gradient force optical trap for dielectric particles. Opt Lett 1986, 11 (5), 288.

2. Ashkin, A.; Dziedzic, J. M., Optical trapping and manipulation of viruses and bacteria. Science 1987, 235 (4795), 1517-20.

3. Block, S. M.; Goldstein, L. S.; Schnapp, B. J., Bead movement by single kinesin molecules studied with optical tweezers. Nature 1990, 348 (6299), 348-52.

4. Hansma, P. K.; Elings, V. B.; Marti, O.; Bracker, C. E., Scanning tunneling microscopy and atomic force microscopy: application to biology and technology. Science 1988, 242 (4876), 20916.

5. Neuman, K. C.; Nagy, A., Single-molecule force spectroscopy: optical tweezers, magnetic tweezers and atomic force microscopy. Nat Methods 2008, 5 (6), 491-505.

6. Willemsen, O. H.; Snel, M. M.; Cambi, A.; Greve, J.; De Grooth, B. G.; Figdor, C. G., Biomolecular interactions measured by atomic force microscopy. Biophys $J$ 2000, 79 (6), 3267-

81.

7. Schaap, I. A.; Carrasco, C.; de Pablo, P. J.; MacKintosh, F. C.; Schmidt, C. F., Elastic response, buckling, and instability of microtubules under radial indentation. Biophys J 2006, 91 (4), 1521-31.

8. Jiao, Y.; Schaffer, T. E., Accurate height and volume measurements on soft samples with the atomic force microscope. Langmuir 2004, 20 (23), 10038-45.

9. Sigdel, K. P.; Pittman, A. E.; Matin, T. R.; King, G. M., High-Resolution AFM-Based Force Spectroscopy. Methods Mol Biol 2018, 1814, 49-62.

10. Matin, T. R.; Sigdel, K. P.; Utjesanovic, M.; Marsh, B. P.; Gallazzi, F.; Smith, V. F.; Kosztin, I.; King, G. M., Single-Molecule Peptide-Lipid Affinity Assay Reveals Interplay between Solution Structure and Partitioning. Langmuir 2017, 33 (16), 4057-4065.

11. Faulk, J. K.; Edwards, D. T.; Bull, M. S.; Perkins, T. T., Improved Force Spectroscopy Using Focused-Ion-Beam-Modified Cantilevers. Methods Enzymol 2017, 582, 321-351.

12. Hutter, J. L. B., J. , Calibration of atomic-force microscope tips. Review of Scientific Instruments 1993, (64), 1868-1873.

13. Bustamante, C.; Macosko, J. C.; Wuite, G. J., Grabbing the cat by the tail: manipulating molecules one by one. Nat Rev Mol Cell Biol 2000, 1 (2), 130-6.

14. Levy, R.; Maaloum, M., Measuring the spring constant of atomic force microscope cantilevers: thermal fluctuations and other methods. Nanotechnology 2002, 13, 33-37.

15. Shaw, J. E.; Epand, R. F.; Hsu, J. C.; Mo, G. C.; Epand, R. M.; Yip, C. M., Cationic peptideinduced remodelling of model membranes: direct visualization by in situ atomic force microscopy. J Struct Biol 2008, 162 (1), 121-38.

16. Pan, J.; Khadka, N. K., Kinetic Defects Induced by Melittin in Model Lipid Membranes: A Solution Atomic Force Microscopy Study. J Phys Chem B 2016, 120 (20), 4625-34.

17. Wiedman, G.; Herman, K.; Searson, P.; Wimley, W. C.; Hristova, K., The electrical response of bilayers to the bee venom toxin melittin: evidence for transient bilayer permeabilization. Biochim Biophys Acta 2013, 1828 (5), 1357-64.

18. Fennouri, A.; Mayer, S. F.; Schroeder, T. B. H.; Mayer, M., Single channel planar lipid bilayer recordings of the melittin variant MelP5. Biochim Biophys Acta 2017.

19. Rakowska, P. D.; Jiang, H.; Ray, S.; Pyne, A.; Lamarre, B.; Carr, M.; Judge, P. J.; Ravi, J.; Gerling, U. I.; Koksch, B.; Martyna, G. J.; Hoogenboom, B. W.; Watts, A.; Crain, J.; Grovenor, C. R.; 
Ryadnov, M. G., Nanoscale imaging reveals laterally expanding antimicrobial pores in lipid bilayers. Proc Natl Acad Sci U S A 2013, 110 (22), 8918-23.

20. Pfeil, M. P.; Pyne, A. L. B.; Losasso, V.; Ravi, J.; Lamarre, B.; Faruqui, N.; Alkassem, H.; Hammond, K.; Judge, P. J.; Winn, M.; Martyna, G. J.; Crain, J.; Watts, A.; Hoogenboom, B. W.; Ryadnov, M. G., Tuneable poration: host defense peptides as sequence probes for antimicrobial mechanisms. Sci Rep 2018, 8 (1), 14926.

21. Pittman, A. E.; Marsh, B. P.; King, G. M., Conformations and Dynamic Transitions of a Melittin Derivative That Forms Macromolecule-Sized Pores in Lipid Bilayers. Langmuir 2018, 34 (28), 8393-8399.

22. Li, S.; Kim, S. Y.; Pittman, A. E.; King, G. M.; Wimley, W. C.; Hristova, K., Potent Macromolecule-Sized Poration of Lipid Bilayers by the Macrolittins, A Synthetically Evolved Family of Pore-Forming Peptides. J Am Chem Soc 2018, 140 (20), 6441-6447.

23. Wiedman, G.; Kim, S. Y.; Zapata-Mercado, E.; Wimley, W. C.; Hristova, K., pH-Triggered, Macromolecule-Sized Poration of Lipid Bilayers by Synthetically Evolved Peptides. J Am Chem Soc 2017, 139 (2), 937-945.

24. Gilbert, R. J. C.; Bayley, H.; Anderluh, G., Membrane pores: from structure and assembly, to medicine and technology. Philos Trans R Soc Lond B Biol Sci 2017, 372 (1726).

25. Brogden, K. A., Antimicrobial peptides: pore formers or metabolic inhibitors in bacteria? Nat Rev Microbiol 2005, 3 (3), 238-50.

26. Soman, N. R.; Baldwin, S. L.; Hu, G.; Marsh, J. N.; Lanza, G. M.; Heuser, J. E.; Arbeit, J. M.; Wickline, S. A.; Schlesinger, P. H., Molecularly targeted nanocarriers deliver the cytolytic peptide melittin specifically to tumor cells in mice, reducing tumor growth. J Clin Invest 2009, 119 (9), 2830-42.

27. Salomone, F.; Cardarelli, F.; Di Luca, M.; Boccardi, C.; Nifosi, R.; Bardi, G.; Di Bari, L.; Serresi, M.; Beltram, F., A novel chimeric cell-penetrating peptide with membrane-disruptive properties for efficient endosomal escape. J Control Release 2012, 163 (3), 293-303.

28. Rausch, J. M.; Marks, J. R.; Rathinakumar, R.; Wimley, W. C., Beta-sheet pore-forming peptides selected from a rational combinatorial library: mechanism of pore formation in lipid vesicles and activity in biological membranes. Biochemistry 2007, 46 (43), 12124-39.

29. Krauson, A. J.; Hall, O. M.; Fuselier, T.; Starr, C. G.; Kauffman, W. B.; Wimley, W. C., Conformational Fine-Tuning of Pore-Forming Peptide Potency and Selectivity. J Am Chem Soc 2015, 137 (51), 16144-52.

30. Li, Y.; Qian, Z.; Ma, L.; Hu, S.; Nong, D.; Xu, C.; Ye, F.; Lu, Y.; Wei, G.; Li, M., Singlemolecule visualization of dynamic transitions of pore-forming peptides among multiple transmembrane positions. Nat Commun 2016, 7, 12906.

31. Chen, F.-Y.; Lee, M.-T.; Huang, H. W., Evidence for Membrane Thinning Effect as the Mechanism for Peptide-Induced Pore Formation. Biophysical Journal 2003, 84 (6), 3751-3758.

32. Hanke, W.; Methfessel, C.; Wilmsen, H. U.; Katz, E.; Jung, G.; Boheim, G., Melittin and a chemically modified trichotoxin form alamethicin-type multi-state pores. Biochim Biophys Acta 1983, 727 (1), 108-14.

33. Fennouri, A.; Mayer, S. F.; Schroeder, T. B. H.; Mayer, M., Single channel planar lipid bilayer recordings of the melittin variant MelP5. Biochim Biophys Acta 2017, 1859 (10), 20512057.

34. Yang, Z.; Choi, H.; Weisshaar, J. C., Melittin-Induced Permeabilization, Re-sealing, and Re-permeabilization of E. coli Membranes. Biophys J 2018, 114 (2), 368-379.

35. Hristova, K.; Dempsey, C. E.; White, S. H., Structure, location, and lipid perturbations of melittin at the membrane interface. Biophys J 2001, 80 (2), 801-11. 
36. van den Bogaart, G.; Guzman, J. V.; Mika, J. T.; Poolman, B., On the mechanism of pore formation by melittin. J Biol Chem 2008, 283 (49), 33854-7.

37. Bechinger, B., The structure, dynamics and orientation of antimicrobial peptides in membranes by multidimensional solid-state NMR spectroscopy. Biochim Biophys Acta 1999, 1462 (1-2), 157-83.

38. Huang, H. W., Molecular mechanism of antimicrobial peptides: the origin of cooperativity. Biochim Biophys Acta 2006, 1758 (9), 1292-302.

39. Wiedman, G.; Fuselier, T.; He, J.; Searson, P. C.; Hristova, K.; Wimley, W. C., Highly Efficient Macromolecule-Sized Poration of Lipid Bilayers by a Synthetically Evolved Peptide. Journal of the American Chemical Society 2014, 136 (12), 4724-4731.

40. Woo, S. Y.; Lee, H., Aggregation and insertion of melittin and its analogue MelP5 into lipid bilayers at different concentrations: effects on pore size, bilayer thickness and dynamics. Phys Chem Chem Phys 2017, 19 (10), 7195-7203.

41. Muller, D. J.; Dufrene, Y. F., Atomic force microscopy: a nanoscopic window on the cell surface. Trends Cell Biol 2011, 21 (8), 461-9.

42. Sanganna Gari, R. R.; Frey, N. C.; Mao, C.; Randall, L. L.; King, G. M., Dynamic Structure of the Translocon SecYEG in Membrane: Direct Single Molecule Observations. J Biol Chem 2013, $288(23), 16848-54$.

43. Cremer, P. S.; Boxer, S. G., Formation and spreading of lipid bilayers on planar glass supports. Journal of Physical Chemistry B 1999, 103 (13), 2554-2559.

44. Chada, N.; Sigdel, K. P.; Gari, R. R.; Matin, T. R.; Randall, L. L.; King, G. M., Glass is a Viable Substrate for Precision Force Microscopy of Membrane Proteins. Sci Rep 2015, 5, 12550.

45. van Meer, G.; Voelker, D. R.; Feigenson, G. W., Membrane lipids: where they are and how they behave. Nat Rev Mol Cell Biol 2008, 9 (2), 112-24.

46. Luckey, M., Membrane Structural Biology. 2nd ed.; Cambridge University Press: 2014.

47. Marsh, B. P.; Chada, N.; Sanganna Gari, R. R.; Sigdel, K. P.; King, G. M., The Hessian Blob Algorithm: Precise Particle Detection in Atomic Force Microscopy Imagery. Sci Rep 2018, 8 (1), 978.

48. King, G. M.; Golovchenko, J. A., Probing nanotube-nanopore interactions. Phys Rev Lett 2005, 95 (21), 216103.

49. Lyu, Y.; Zhu, X.; Xiang, N.; Narsimhan, G., Molecular Dynamics Study of Pore Formation by Melittin in a 1,2-Dioleoyl-sn-glycero-3-phosphocholine and 1,2-Di(9Z-octadecenoyl)-snglycero-3-phospho-(1'-rac-glycerol) Mixed Lipid Bilayer. Industrial \& Engineering Chemistry Research 2015, 54 (42), 10275-10283.

50. Mingeot-Leclercq, M. P.; Deleu, M.; Brasseur, R.; Dufrene, Y. F., Atomic force microscopy of supported lipid bilayers. Nat Protoc 2008, 3 (10), 1654-9.

51. Wang, Z.; Wang, G., APD: the Antimicrobial Peptide Database. Nucleic Acids Res 2004, 32 (Database issue), D590-2.

52. Hancock, R. E.; Sahl, H. G., Antimicrobial and host-defense peptides as new anti-infective therapeutic strategies. Nat Biotechnol 2006, 24 (12), 1551-7.

53. Pan, H.; Soman, N. R.; Schlesinger, P. H.; Lanza, G. M.; Wickline, S. A., Cytolytic peptide nanoparticles ('NanoBees') for cancer therapy. Wiley Interdiscip Rev Nanomed Nanobiotechnol 2011, 3 (3), 318-27.

54. Hood, J. L.; Jallouk, A. P.; Campbell, N.; Ratner, L.; Wickline, S. A., Cytolytic nanoparticles attenuate HIV-1 infectivity. Antivir Ther 2013, 18 (1), 95-103.

55. Gerlach, S. L.; Rathinakumar, R.; Chakravarty, G.; Goransson, U.; Wimley, W. C.; Darwin, S. P.; Mondal, D., Anticancer and chemosensitizing abilities of cycloviolacin 02 from Viola odorata and psyle cyclotides from Psychotria leptothyrsa. Biopolymers 2010, 94 (5), 617-25. 
56. Suhorutsenko, J.; Oskolkov, N.; Arukuusk, P.; Kurrikoff, K.; Eriste, E.; Copolovici, D. M.; Langel, U., Cell-penetrating peptides, PepFects, show no evidence of toxicity and immunogenicity in vitro and in vivo. Bioconjug Chem 2011, 22 (11), 2255-62.

57. Bukovnik, U.; Gao, J.; Cook, G. A.; Shank, L. P.; Seabra, M. B.; Schultz, B. D.; Iwamoto, T.; Chen, J.; Tomich, J. M., Structural and biophysical properties of a synthetic channel-forming peptide: designing a clinically relevant anion selective pore. Biochim Biophys Acta 2012, 1818 (4), 1039-48.

58. Krauson, A. J.; He, J.; Wimley, W. C., Determining the mechanism of membrane permeabilizing peptides: identification of potent, equilibrium pore-formers. Biochim Biophys Acta 2012, 1818 (7), 1625-32.

59. Wimley, W. C., How Does Melittin Permeabilize Membranes? Biophys J 2018, 114 (2), 251-253.

60. Krauson, A. J.; He, J.; Wimley, A. W.; Hoffmann, A. R.; Wimley, W. C., Synthetic molecular evolution of pore-forming peptides by iterative combinatorial library screening. ACS Chem Biol 2013, 8 (4), 823-31.

61. Krauson, A. J.; He, J.; Wimley, W. C., Gain-of-function analogues of the pore-forming peptide melittin selected by orthogonal high-throughput screening. J Am Chem Soc 2012, 134 (30), 12732-41.

62. Marks, J. R.; Placone, J.; Hristova, K.; Wimley, W. C., Spontaneous membranetranslocating peptides by orthogonal high-throughput screening. J Am Chem Soc 2011, 133 (23), 8995-9004.

63. Rathinakumar, R.; Wimley, W. C., High-throughput discovery of broad-spectrum peptide antibiotics. FASEB J 2010, 24 (9), 3232-8.

64. Rathinakumar, R.; Wimley, W. C., Biomolecular engineering by combinatorial design and high-throughput screening: small, soluble peptides that permeabilize membranes. J Am Chem Soc 2008, 130 (30), 9849-58.

65. Rausch, J. M.; Marks, J. R.; Wimley, W. C., Rational combinatorial design of pore-forming beta-sheet peptides. Proc Natl Acad Sci U S A 2005, 102 (30), 10511-5.

66. Stewart, J. C., Colorimetric determination of phospholipids with ammonium ferrothiocyanate. Anal Biochem 1980, 104 (1), 10-4.

67. Wimley, W. C.; Selsted, M. E.; White, S. H., Interactions between human defensins and lipid bilayers: evidence for formation of multimeric pores. Protein Sci 1994, 3 (9), 1362-73.

68. Terwilliger, T. C.; Weissman, L.; Eisenberg, D., The structure of melittin in the form I crystals and its implication for melittin's lytic and surface activities. Biophys J 1982, 37 (1), 35361.

69. Ladokhin, A. S.; Jayasinghe, S.; White, S. H., How to measure and analyze tryptophan fluorescence in membranes properly, and why bother? Anal Biochem 2000, 285 (2), 235-45. 70. Bechinger, B., Structure and functions of channel-forming peptides: magainins, cecropins, melittin and alamethicin. J Membr Biol 1997, 156 (3), 197-211.

71. Frey, S.; Tamm, L. K., Orientation of melittin in phospholipid bilayers. A polarized attenuated total reflection infrared study. Biophys J 1991, 60 (4), 922-30.

72. Gazzara, J. A.; Phillips, M. C.; Lund-Katz, S.; Palgunachari, M. N.; Segrest, J. P.; Anantharamaiah, G. M.; Snow, J. W., Interaction of class A amphipathic helical peptides with phospholipid unilamellar vesicles. J Lipid Res 1997, 38 (10), 2134-46.

73. De Angelis, A. A.; Opella, S. J., Bicelle samples for solid-state NMR of membrane proteins. Nat Protoc 2007, 2 (10), 2332-8. 
74. Epand, R. M.; Shai, Y.; Segrest, J. P.; Anantharamaiah, G. M., Mechanisms for the modulation of membrane bilayer properties by amphipathic helical peptides. Biopolymers 1995, 37 (5), 319-38.

75. Vargas, C.; Arenas, R. C.; Frotscher, E.; Keller, S., Nanoparticle self-assembly in mixtures of phospholipids with styrene/maleic acid copolymers or fluorinated surfactants. Nanoscale 2015, 7 (48), 20685-96.

76. Bechinger, B., Towards membrane protein design: $\mathrm{pH}$-sensitive topology of histidinecontaining polypeptides. J Mol Biol 1996, 263 (5), 768-75.

77. Sengupta, D.; Leontiadou, H.; Mark, A. E.; Marrink, S. J., Toroidal pores formed by antimicrobial peptides show significant disorder. Biochim Biophys Acta 2008, 1778 (10), 2308-

17.

78. Allende, D.; Simon, S. A.; McIntosh, T. J., Melittin-induced bilayer leakage depends on lipid material properties: evidence for toroidal pores. Biophys J 2005, 88 (3), 1828-37.

79. Yang, L.; Harroun, T. A.; Weiss, T. M.; Ding, L.; Huang, H. W., Barrel-stave model or toroidal model? A case study on melittin pores. Biophys $J \mathbf{2 0 0 1 , 8 1}(3), 1475-85$.

80. Nishimura, Y.; Takeda, K.; Ezawa, R.; Ishii, J.; Ogino, C.; Kondo, A., A display of pHsensitive fusogenic GALA peptide facilitates endosomal escape from a Bio-nanocapsule via an endocytic uptake pathway. J Nanobiotechnology 2014, 12, 11.

81. Moulay, G.; Leborgne, C.; Mason, A. J.; Aisenbrey, C.; Kichler, A.; Bechinger, B., Histidinerich designer peptides of the LAH4 family promote cell delivery of a multitude of cargo. $J$ Pept Sci 2017, 23 (4), 320-328.

82. Yao, L.; Daniels, J.; Wijesinghe, D.; Andreev, O. A.; Reshetnyak, Y. K., pHLIP(R)-mediated delivery of PEGylated liposomes to cancer cells. J Control Release 2013, 167 (3), 228-37.

83. Andreev, O. A.; Engelman, D. M.; Reshetnyak, Y. K., pH-sensitive membrane peptides (pHLIPs) as a novel class of delivery agents. Mol Membr Biol 2010, 27 (7), 341-52.

84. An, M.; Wijesinghe, D.; Andreev, O. A.; Reshetnyak, Y. K.; Engelman, D. M., pH-(low)insertion-peptide ( $\mathrm{pHLIP}$ ) translocation of membrane impermeable phalloidin toxin inhibits cancer cell proliferation. Proc Natl Acad Sci U S A 2010, 107 (47), 20246-50.

85. Parente, R. A.; Nir, S.; Szoka, F. C., Jr., Mechanism of leakage of phospholipid vesicle contents induced by the peptide GALA. Biochemistry 1990, 29 (37), 8720-8.

86. Fendos, J.; Barrera, F. N.; Engelman, D. M., Aspartate embedding depth affects pHLIP's insertion pKa. Biochemistry 2013, 52 (27), 4595-604.

87. Wiedman, G.; Wimley, W. C.; Hristova, K., Testing the limits of rational design by engineering pH sensitivity into membrane-active peptides. Biochim Biophys Acta 2015, 1848 (4), 951-7.

88. Bohrer, M. P.; Deen, W. M.; Robertson, C. R.; Troy, J. L.; Brenner, B. M., Influence of molecular configuration on the passage of macromolecules across the glomerular capillary wall. J Gen Physiol 1979, 74 (5), 583-93.

89. Hunt, J. F.; Rath, P.; Rothschild, K. J.; Engelman, D. M., Spontaneous, pH-dependent membrane insertion of a transbilayer alpha-helix. Biochemistry 1997, 36 (49), 15177-92.

90. Ladokhin, A. S.; Wimley, W. C.; Hristova, K.; White, S. H., Mechanism of leakage of contents of membrane vesicles determined by fluorescence requenching. Methods Enzymol 1997, 278, 474-86.

91. Hacke, U. G.; Sperry, J. S.; Pittermann, J., Analysis of circular bordered pit function II. Gymnosperm tracheids with torus-margo pit membranes. Am J Bot 2004, 91 (3), 386-400.

92. Kaiser, E. T.; Kezdy, F. J., Secondary structures of proteins and peptides in amphiphilic environments. (A review). Proc Natl Acad Sci U S A 1983, 80 (4), 1137-43. 
93. Sani, M. A.; Separovic, F., How Membrane-Active Peptides Get into Lipid Membranes. Acc Chem Res 2016, 49 (6), 1130-8.

94. Ladokhin, A. S., Distribution analysis of depth-dependent fluorescence quenching in membranes: a practical guide. Methods Enzymol 1997, 278, 462-73.

95. Wimley, W. C.; Hristova, K.; Ladokhin, A. S.; Silvestro, L.; Axelsen, P. H.; White, S. H., Folding of beta-sheet membrane proteins: a hydrophobic hexapeptide model. J Mol Biol 1998, 277 (5), 1091-110.

96. Wimley, W. C., Describing the mechanism of antimicrobial peptide action with the interfacial activity model. ACS Chem Biol 2010, 5 (10), 905-17.

97. White, S. H.; Wimley, W. C., Membrane protein folding and stability: physical principles. Annu Rev Biophys Biomol Struct 1999, 28, 319-65.

98. White, S. H.; Wimley, W. C.; Ladokhin, A. S.; Hristova, K., Protein folding in membranes: determining energetics of peptide-bilayer interactions. Methods Enzymol 1998, 295, 62-87.

99. Wimley, W. C.; White, S. H., Experimentally determined hydrophobicity scale for proteins at membrane interfaces. Nat Struct Biol 1996, 3 (10), 842-8.

100. Wu, Y.; Huang, H. W.; Olah, G. A., Method of oriented circular dichroism. Biophys J 1990, 57 (4), 797-806.

101. Hristova, K.; Wimley, W. C.; Mishra, V. K.; Anantharamiah, G. M.; Segrest, J. P.; White, S. $\mathrm{H}$., An amphipathic alpha-helix at a membrane interface: a structural study using a novel X-ray diffraction method. J Mol Biol 1999, 290 (1), 99-117.

102. Muller, D. J., AFM: a nanotool in membrane biology. Biochemistry 2008, 47 (31), $7986-$

98.

103. Rief, M.; Gautel, M.; Oesterhelt, F.; Fernandez, J. M.; Gaub, H. E., Reversible unfolding of individual titin immunoglobulin domains by AFM. Science 1997, 276 (5315), 1109-12.

104. Borgia, A.; Williams, P. M.; Clarke, J., Single-molecule studies of protein folding. Annu Rev Biochem 2008, 77, 101-25.

105. Kim, B. H.; Lyubchenko, Y. L., Nanoprobing of misfolding and interactions of amyloid beta 42 protein. Nanomedicine : nanotechnology, biology, and medicine 2014, 10 (4), 871-8.

106. Oesterhelt, F.; Oesterhelt, D.; Pfeiffer, M.; Engel, A.; Gaub, H. E.; Müller, D. J., Unfolding pathways of individual bacteriorhodopsins. Science 2000, 288 (5463), 143-6.

107. Bippes, C.; Müller, D., High-resolution atomic force microscopy and spectroscopy of native membrane proteins Rep. Prog. Phys. 2011, 74, 086601.

108. Petrosyan, R.; Bippes, C. A.; Walheim, S.; Harder, D.; Fotiadis, D.; Schimmel, T.; Alsteens, D.; Muller, D. J., Single-molecule force spectroscopy of membrane proteins from membranes freely spanning across nanoscopic pores. Nano letters 2015, 15 (5), 3624-33.

109. Yu, H.; Siewny, M. G.; Edwards, D. T.; Sanders, A. W.; Perkins, T. T., Hidden dynamics in the unfolding of individual bacteriorhodopsin proteins. Science 2017, 355 (6328), 945-950.

110. Ganchev, D. N.; Rijkers, D. T.; Snel, M. M.; Killian, J. A.; de Kruijff, B., Strength of integration of transmembrane alpha-helical peptides in lipid bilayers as determined by atomic force spectroscopy. Biochemistry 2004, 43 (47), 14987-93.

111. Desmeules, P.; Grandbois, M.; Bondarenko, V. A.; Yamazaki, A.; Salesse, C., Measurement of membrane binding between recoverin, a calcium-myristoyl switch protein, and lipid bilayers by AFM-based force spectroscopy. Biophysical journal 2002, 82 (6), 3343-50.

112. Andre, G.; Brasseur, R.; Dufrene, Y. F., Probing the interaction forces between hydrophobic peptides and supported lipid bilayers using AFM. Journal of molecular recognition : JMR 2007, 20 (6), 538-45. 
113. Schwierz, N.; Krysiak, S.; Hugel, T.; Zacharias, M., Mechanism of Reversible PeptideBilayer Attachment: Combined Simulation and Experimental Single-Molecule Study. Langmuir : the ACS journal of surfaces and colloids 2016, 32 (3), 810-21.

114. Churnside, A. B.; Sullan, R. M.; Nguyen, D. M.; Case, S. O.; Bull, M. S.; King, G. M.; Perkins, T. T., Routine and timely sub-picoNewton force stability and precision for biological applications of atomic force microscopy. Nano letters 2012, 12 (7), 3557-61.

115. Edwards, D. T.; Faulk, J. K.; Sanders, A. W.; Bull, M. S.; Walder, R.; LeBlanc, M. A.; Sousa, M. C.; Perkins, T. T., Optimizing 1-mus-Resolution Single-Molecule Force Spectroscopy on a Commercial Atomic Force Microscope. Nano letters 2015, 15 (10), 7091-8.

116. Walder, R.; LeBlanc, M. A.; Van Patten, W. J.; Edwards, D. T.; Greenberg, J. A.; Adhikari, A.; Okoniewski, S. R.; Sullan, R. M. A.; Rabuka, D.; Sousa, M. C.; Perkins, T. T., Rapid Characterization of a Mechanically Labile alpha-Helical Protein Enabled by Efficient Site-Specific Bioconjugation. J Am Chem Soc 2017, 139 (29), 9867-9875.

117. Faulk, J. K.; Edwards, D. T.; Bull, M. S.; Perkins, T. T., Improved Force Spectroscopy Using Focused-Ion-Beam-Modified Cantilevers. Methods in Enzymology 2017, 582, 321.

118. Edwards, D. T.; Perkins, T. T., Optimizing force spectroscopy by modifying commercial cantilevers: Improved stability, precision, and temporal resolution. J Struct Biol 2017, 197 (1), 13-25.

119. Viani, M. B.; Schäffer, T. E.; Chand, A.; Rief, M.; Gaub, H. E.; Hansma, P. K., Small cantilevers for force spectroscopy of single molecules. Journal of Applied Physics 1999, 86 (4), 2258-2262.

120. Bull, M. S.; Sullan, R. M.; Li, H.; Perkins, T. T., Improved single molecule force spectroscopy using micromachined cantilevers. ACS Nano 2014, 8 (5), 4984-95.

121. El Kirat, K.; Morandat, S.; Dufrene, Y. F., Nanoscale analysis of supported lipid bilayers using atomic force microscopy. Biochim Biophys Acta 2010, 1798 (4), 750-65.

122. Richter, R. P.; Brisson, A. R., Following the formation of supported lipid bilayers on mica: a study combining AFM, QCM-D, and ellipsometry. Biophys J 2005, 88 (5), 3422-33.

123. Hamai, C.; Cremer, P. S.; Musser, S. M., Single giant vesicle rupture events reveal multiple mechanisms of glass-supported bilayer formation. Biophys J 2007, 92 (6), 1988-99.

124. Vedder, W., Correlations between infrared spectrum and chemical composition of mica. American Mineralogist 1964, 49 (5-6), 736-768.

125. Cho, N. J.; Dvory-Sobol, H.; Xiong, A.; Cho, S. J.; Frank, C. W.; Glenn, J. S., Mechanism of an amphipathic alpha-helical peptide's antiviral activity involves size-dependent virus particle lysis. ACS Chem Biol 2009, 4 (12), 1061-7.

126. Jackman, J. A.; Costa, V. V.; Park, S.; Real, A.; Park, J. H.; Cardozo, P. L.; Ferhan, A. R.; Olmo, I. G.; Moreira, T. P.; Bambirra, J. L.; Queiroz, V. F.; Queiroz-Junior, C. M.; Foureaux, G.; Souza, D. G.; Ribeiro, F. M.; Yoon, B. K.; Wynendaele, E.; De Spiegeleer, B.; Teixeira, M. M.; Cho, N. J., Therapeutic treatment of Zika virus infection using a brain-penetrating antiviral peptide. Nat Mater 2018, 17 (11), 971-977.

127. Jackman, J. A.; Saravanan, R.; Zhang, Y.; Tabaei, S. R.; Cho, N. J., Correlation between Membrane Partitioning and Functional Activity in a Single Lipid Vesicle Assay Establishes Design Guidelines for Antiviral Peptides. Small 2015, 11 (20), 2372-9.

128. Schmitz, I.; Schreiner, M.; Friedbacher, G.; Grasserbauer, M., Phase imaging as an extension to tapping mode AFM for the identification of material properties on humiditysensitive surfaces. Applied Surface Science 1997, 115 (2), 190-198.

129. Proksch, R.; Kocun, M.; Hurley, D.; Viani, M.; Labuda, A.; Meinhold, W.; Bemis, J., Practical loss tangent imaging with amplitude-modulated atomic force microscopy. Journal of Applied Physics 2016, 119 (13), 134901. 
130. Stark, M.; Moller, C.; Muller, D. J.; Guckenberger, R., From images to interactions: highresolution phase imaging in tapping-mode atomic force microscopy. Biophys $J \mathbf{2 0 0 1}, 80$ (6), 3009-18.

131. Nagao, E.; Dvorak, J. A., Phase imaging by atomic force microscopy: analysis of living homoiothermic vertebrate cells. Biophys J 1999, 76 (6), 3289-97. 


\section{Vita}

Anna Pittman was born in Jonesboro, Arkansas. In 2015 she graduated with a Bachelor of Arts degree in Physics from Hendrix College in Conway, Arkansas. Following that she moved to Columbia, Missouri and obtained a Master of Science degree in Physics while pursuing her PhD. Working in the laboratory of Dr. Gavin King, she received her Doctor of Philosophy in Physics in May of 2019. Anna will work next at St. Jude Children's Research Hospital in Memphis, Tennessee, in the laboratory of Dr. David Solecki in the department of developmental neurobiology. 